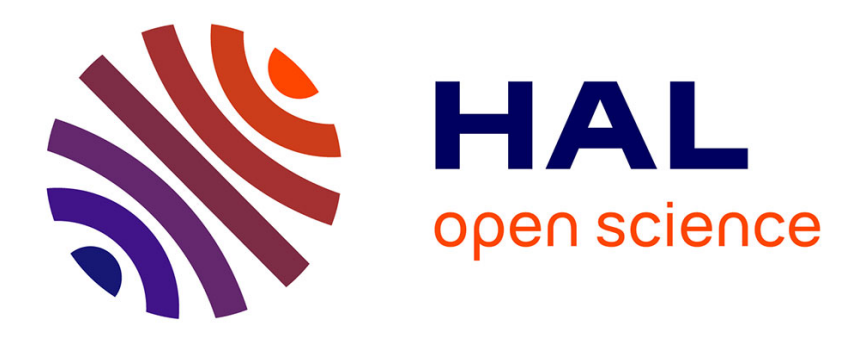

\title{
Oseen resolvent estimates with small resolvent parameter
}

Paul Deuring

\section{- To cite this version:}

| Paul Deuring. Oseen resolvent estimates with small resolvent parameter. 2017. hal-01534690

\author{
HAL Id: hal-01534690 \\ https://hal.science/hal-01534690
}

Preprint submitted on 8 Jun 2017

HAL is a multi-disciplinary open access archive for the deposit and dissemination of scientific research documents, whether they are published or not. The documents may come from teaching and research institutions in France or abroad, or from public or private research centers.
L'archive ouverte pluridisciplinaire HAL, est destinée au dépôt et à la diffusion de documents scientifiques de niveau recherche, publiés ou non, émanant des établissements d'enseignement et de recherche français ou étrangers, des laboratoires publics ou privés. 


\title{
Oseen resolvent estimates with small resolvent parameter.
}

\author{
Paul Deuring \\ Université du Littoral Côte d'Opale, Laboratoire de Mathématiques Pures et Appliquées \\ Joseph Liouville, F-62228 Calais, France.
}

\begin{abstract}
We consider the Oseen system with resolvent term in an exterior domain in $\mathbb{R}^{3}$, supplemented by homogeneous Dirichlet boundary conditions. Under the assumption that the resolvent parameter $\lambda$ is close to zero and $\Re \lambda \geq 0, \lambda \neq 0$, we estimate the $L^{p_{-}}$ norm of the velocity against the $L^{p}$-norm of the right-hand side, times a factor $C|\lambda|^{-2}$, with $C>0$ independent of $\lambda$. Such an estimate cannot hold for this range of $\lambda$ if $|\lambda|^{-2}$ is replaced by $|\lambda|^{-\kappa}$ with $\kappa<3 / 2$, and there are indications that $\kappa \in[3 / 2,2)$ cannot be admitted either. We present various other $L^{p}$-estimates of Oseen resolvent flows for the same range of $\lambda$. Our article is complementary to the work by T. Kobayashi and Y. Shibata, On the Oseen equation in the three dimensional exterior domains, Math. Ann. 310 (1998), 1-45, where Oseen resolvent estimates are derived under the assumption that $|\lambda| \geq c_{0}$, for some arbitrary but fixed $c_{0}>0$, with the constant in the resolvent estimate depending on $c_{0}$.
\end{abstract}

AMS subject classifications. 35Q30, 65N30, 76D05.

Key words. Incompressible Navier-Stokes system, Oseen term, resolvent estimate.

\section{Introduction}

An incompressible viscous flow around a rigid body moving steadily and without rotation is usually described by the Navier-Stokes system with an Oseen term,

$$
\begin{gathered}
\partial_{t} u(x, t)-\Delta_{x} u(x, t)+\tau \partial_{x_{1}} u(x, t)+\tau\left(u(x, t) \cdot \nabla_{x}\right) u(x, t)+\nabla_{x} \pi(x, t)=f(x, t),(1.1) 1 \\
\operatorname{div}_{x} u(x, t)=0 \text { for } t \in(0, \infty), x \in \bar{\Omega}^{c}:=\mathbb{R}^{3} \backslash \bar{\Omega},
\end{gathered}
$$

where the open, bounded set $\Omega \subset \mathbb{R}^{3}$ represents the rigid body, and $\tau \in(0, \infty)$ is the Reynolds number. The unknowns of this system are the velocity field $u$ and the pressure field $\pi$ of the fluid. The velocity in question is the "velocity above ground". This means that the fluid particle located at point $x$ at time $t$ moves with velocity $u(x, t)$ with respect to a fixed point in space, where $x_{1}, x_{2}, x_{3}$ are the coordinates of the particle with respect to a reference system which adheres to the rigid body, and so is not attached to the fixed point in space. This type of velocity has two advantages from a mathematical point of view. Firstly, the rigid body is represented by a set that does not depend on time, and secondly, the velocity of the fluid vanishes at infinity in the sense that $u(x, t) \rightarrow 0$ for $|x| \rightarrow \infty$, a condition that may be expressed in weak form by the relation $u(\cdot, t) \in L^{r}\left(\bar{\Omega}^{c}\right)^{3}$ for $t \in(0, \infty)$, where $r$ is some number in $[1, \infty)$. But on the other hand, an additional term, that is, the Oseen term $\tau \partial_{x_{1}} u(x, t)$, arises in system (1.1), complicating some aspects of the mathematical theory related to (1.1). In the work at hand, we will deal with such an aspect. In fact, we consider the Oseen resolvent system

$$
-\Delta U+\tau \partial_{1} U+\lambda U+\nabla \Pi=G, \operatorname{div} U=0 \quad \text { in } \bar{\Omega}^{c},
$$


as well as the stationary Oseen system

$$
-\Delta U+\tau \partial_{1} U+\nabla \Pi=G, \operatorname{div} U=0 \quad \text { in } \bar{\Omega}^{c},
$$

which may be considered as a special case of $(1.2)(\lambda=0)$. In order to explain what we want to show with respect to (1.2) and (1.3), let us first recall some facts about the Stokes resolvent system

$$
-\Delta U+\lambda U+\nabla \Pi=G, \operatorname{div} U=0 \quad \text { in } \bar{\Omega}^{c},
$$

under Dirichlet boundary conditions

$$
u \mid \partial \Omega=0 .
$$

If $S \in(0, \infty)$ with $\bar{\Omega} \subset B_{S}, p \in(1, \infty)$ and $\vartheta \in(\pi / 2, \pi)$, then for any $\lambda \in \mathbb{C} \backslash\{0\}$ with $|\arg \lambda| \leq \vartheta$, there is a unique pair of functions $(U, \Pi)$ such that $U \in W^{2, p}\left(\bar{\Omega}^{c}\right)^{3} \cap$ $W_{0}^{1, p}\left(\bar{\Omega}^{c}\right)^{3}, \Pi \in W_{l o c}^{1, p}\left(\bar{\Omega}^{c}\right), \nabla \Pi \in L^{p}\left(\bar{\Omega}^{c}\right)^{3}$ and $\int_{B_{S} \backslash \bar{\Omega}} \Pi d x=0$, and such that (1.4) is satisfied. In addition, there is a constant $C>0$, only depending on $\Omega, p$ and $\vartheta$, such that

$$
\|U\|_{p} \leq C|\lambda|^{-1}\|G\|_{p} \text { for } \lambda, G \text { and } U \text { as above; }
$$

see [18], [3] or [5], [6], [7] or [25]. Inequality (1.6) is a basic tool in the mathematical study of the Navier-Stokes system

$$
\begin{aligned}
& \partial_{t} u(x, t)-\Delta_{x} u(x, t)+\tau\left(u(x, t) \cdot \nabla_{x}\right) u(x, t)+\nabla_{x} \pi(x, t)=f(x, t), \\
& \operatorname{div}_{x} u(x, t)=0 \quad\left(t \in(0, \infty), x \in \bar{\Omega}^{c}\right),
\end{aligned}
$$

and is applied directly or indirectly in a very large number of papers dealing with this system, usually in the context of the theory of analytic semigroups.

It is well known that the existence result stated above for solutions to (1.4), (1.5) does not remain valid in the Oseen case, nor does inequality (1.6) for the same range of $\lambda$. In fact, it was shown in [2] and [14, Theorem 3.1] that if $\tau^{2} \Re \lambda \leq-(\Im \lambda)^{2}$, then there are some functions $G \in L^{p}\left(\bar{\Omega}^{c}\right)^{3}$ such that a solution to (1.2), (1.5) need not exist or be unique, no matter what is the choice of $p \in(1, \infty)$. As a consequence, since the set $\left\{\lambda \in \mathbb{C} \backslash\{0\}: \tau^{2} \Re \lambda \leq-(\Im \lambda)^{2}\right\}$ intersects the sector $\{\lambda \in \mathbb{C} \backslash\{0\}:|\arg \lambda| \leq \vartheta\}$ for any $\vartheta \in(\pi / 2, \pi)$, inequality (1.6) indeed cannot be valid for the same range of $\lambda$ as in the Stokes case. Therefore it may be asked to which extent estimate (1.6) carries over to solutions of the Oseen resolvent system (1.2) under Dirichlet boundary conditions (1.5). Answers to this question determine how semigroup theory may be used in order to study solutions to (1.1).

As far as we know, the most detailed results up to now about existence of solutions to (1.2), (1.5) and about estimates of these solutions are due to Kobayashi, Shibata [19]. According to [19, Theorem 4.4], for any $\lambda \in \mathbb{C} \backslash\{0\}$ with $\Re \lambda \geq 0, p \in(1, \infty)$ and $G \in L^{p}\left(\bar{\Omega}^{c}\right)^{3}$, there is a unique solution $(U, \Pi)$ to boundary value problem $(1.2),(1.5)$, where the notion of "solution" is to be understood in the same sense as described above in the context of (1.4), (1.5). Let us denote the velocity part $U$ of this solution by $\mathfrak{V}(\lambda, G)$. Then, again by [19, Theorem 4.4], for any $p \in(1, \infty)$ and $C_{0} \in(0, \infty)$, there is a constant $C>0$ depending 
on $\Omega, p$ and $C_{0}$, such that inequality (1.6) is valid for $G \in L^{p}\left(\bar{\Omega}^{c}\right)^{3}, \lambda \in \mathbb{C}$ with $\Re \lambda \geq 0$ and $|\lambda| \geq C_{0}$, and for $\mathfrak{V}(\lambda, G)$ in the place of $U$.

This raises the question as to whether estimate (1.6) remains valid when $|\lambda|$ tends to 0 in the right half of the complex plane. A negative result in this respect was derived in [12]. It states that even if $p=2$ and $\Omega=\emptyset\left(\right.$ hence $\bar{\Omega}^{c}=\mathbb{R}^{3}$ ), there cannot be a constant $C>0$ and an exponent $\kappa \in[0,3 / 2)$ such that

$$
\|\mathfrak{V}(\lambda, G)\|_{p} \leq C|\lambda|^{-\kappa}\|G\|_{p}
$$

for any $G \in L^{p}\left(\bar{\Omega}^{c}\right)^{3}, \lambda \in \mathbb{C} \backslash\{0\}$ with $\Re \lambda \geq 0$ and $|\lambda| \leq 1$. The condition $|\lambda| \leq 1$ may be replaced by $|\lambda| \leq M$ for any $M>0$, as follows from [19, Theorem 4.4].

It is the aim of the present article to prove a positive result in this respect, that is, estimates of solutions to (1.2), (1.5) remaining valid when $|\lambda|$ tends to zero, provided that $\Re \lambda \geq 0$. In particular we will show that inequality (1.7) holds with $\kappa=2$ for any $p \in(1, \infty)$ and any $\lambda \in \mathbb{C} \backslash\{0\}$ with $\Re \lambda \geq 0$ and $|\lambda| \leq(\tau / 2)^{2}$; see Theorem 6.4. In view of [19, Theorem 4.4], the quantity $(\tau / 2)^{2}$ can be replaced by any constant $M>0$. Of course, the negative result in [12] does not exclude that inequality (1.7) holds even for some $\kappa \in[3 / 2,2)$. However, we will derive our estimate by reducing it to an analogous inequality in the whole space $\mathbb{R}^{3}$ under the assumption $p \leq 2$; see the proof of Theorem 6.1. This latter inequality seems to be sharp as concerns the exponent $\kappa=2$, so we would conjecture that the same is true for (1.7). In addition to (1.7), we will show various other estimates of solutions to (1.2), (1.5), some of them involving upper bounds independent of $\lambda$, and then being valid for solutions to the Oseen system (1.3) as well. We refer to Theorem 6.1, 6.3 and 6.4 for details.

As concerns interesting applications of our results, we mention a companion paper [9], which actually motivated the work at hand, and which deals with stability of solutions to the stationary Navier-Stokes system with Oseen term,

$$
-\Delta U+\tau \partial_{1} U+\tau(U \cdot \nabla) U+\nabla \Pi=G, \operatorname{div} U=0 \quad \text { in } \bar{\Omega}^{c},
$$

under the assumptions that all eigenvalues of a certain linear operator have negative real part. Some of our estimates from Theorem 6.1 and 6.3 constitute key elememts of the theory developed in that reference.

Let us indicate how we proceed in order to prove our results. In section 2 , we collect various auxiliary results related to subjects like, for example, the Helmholtz decomposition, the divergence equation $\operatorname{div} V=F$, and the decay behaviour of functions $V \in W_{l o c}^{1,1}\left(\bar{\Omega}^{c}\right)$ with $\nabla V \in L^{p}\left(\bar{\Omega}^{c}\right)^{3}$ for some $p \in(1,3)$. These results are essentially well known, so in order to prove them, we limit ourselves to giving references, except for some details which we think are not so well documented in literature. Section 3 recalls known results about differential equation (1.2), (1.3) or (1.4). Concerning proofs, we are guided by the same principle as in section 2: we cite references, additionally elaborating some details if this seems helpful for the reader. The subject of Section 4 are $L^{p}$-estimates of solutions to the Oseen resolvent system or to the Oseen system in the whole space $\mathbb{R}^{3}$. As our main tool in this context, we represent these solutions by a convolution integral involving a fundamental solution of the scalar Oseen resolvent equation $-\Delta v+\tau \partial_{1} v+\lambda v=h$ or the scalar Oseen equation $-\Delta v+\tau \partial_{1} v=h$. Section 5 provides uniqueness results for solutions of the Oseen resolvent system and the Oseen system, respectively, first in the whole space case (Theorem 5.1), 
and then in the exterior domain case under Dirichlet boundary conditions (1.5) (Theorem 5.2). Although we only slightly generalize existing uniqueness results, a considerable effort is necessary to achieve this generalization. Finally, in section 6 , we turn to $L^{p}$-estimates of solutions to boundary value problem (1.2), (1.5) (Oseen resolvent system) and (1.3), (1.5) (Oseen system). Our general approach consists in reducing the estimates in question to the whole space case and the bounded domain case. This approach was applied in [20] to the Stokes system, in [3] to the Stokes resolvent problem (1.4), (1.5), and in [19] to problem (1.2), (1.5). The reasoning we use in order to make this method work in our context is rather technical. This should be expected, however, because $L^{p}$-estimates of solutions to the Oseen resolvent system, with upper bounds depending on $\lambda$ in an explicit way or being independent of $\lambda$, are difficult to come by. We recall in this context that estimate (1.6) of the Stokes resolvent is not easily accessible either.

In Theorem 6.1 and 6.4 , solutions to problem (1.2) are estimated in the form $\|U\|_{p_{1}} \leq$ $\mathfrak{C}\|G\|_{q},\|\nabla U\|_{p_{2}} \leq \mathfrak{C}\|G\|_{q}$ and $\|U\|_{p_{3}} \leq \mathfrak{C}|\lambda|^{\kappa}\|G\|_{q}$, with constants $\mathfrak{C}$ independent of $\lambda$ and certain exponents $\kappa$, for a certain range of $q, p_{1}, p_{2}$ and $p_{3}$. Actually a larger range of values for $p_{1}, p_{2}$ and $p_{3}$ may be admitted. This is because, firstly, the approach by duality and interpolation in the proof of Theorem 6.4 may be applied in the case $p_{3} \neq q$ as well, and secondly, because [11, Theorem 10], dealing with the whole space case and reproduced as Theorem 4.1 below, can be extended to certain limit values of $p_{1}$ and $p_{2}$ by applying the Hardy-Littlewood-Sobolev inequality instead of Young's inequality in the reasoning presented in [11]. That latter substitution was already used in the case $p_{1}=6, p_{2}=2, q=6 / 5$, which is addressed in [11, Theorem 10] and Theorem 6.1. However, as concerns the inequality $\left\|D^{2} U\right\|_{q} \leq \mathfrak{C}\|G\|_{q}$, which we only proved for $q=2$ in the case $\lambda \neq 0$ (see Theorem 6.1), we have doubts that it may be extended to a larger range of $q$, in spite of a remark to the contrary in [19, beginning of the proof of Lemma 3.4]. In fact, it is not clear how to come by such an estimate in the whole space case (proof of Theorem 4.2). As a consequence of this situation, we could not extend Theorem 6.3 to cover an inequality of the form $\|\nabla U\|_{q} \leq \mathfrak{C}\|G\|_{-1, q}$ for some $q \neq 2$ if $\lambda \neq 0$ because such an estimate reduces to an inequality of the form $\left\|D^{2} U\right\|_{q} \leq \mathfrak{C}\|G\|_{q}$ (proof of Theorem $4.3)$.

Let us introduce some notation. The set $\Omega$ and the parameter $\tau \in(0, \infty)$ introduced in Section 1 will be kept fixed throughout. Recall that $\Omega$ is supposed to be open and bounded, with $C^{2}$-boundary and with $\bar{\Omega}^{c}$ connected. Put $K_{\tau}:=\{\lambda \in \mathbb{C}: \Re \lambda \geq 0,|\lambda| \leq$ $\left.(\tau / 2)^{2}\right\}$. For $R \in(0, \infty)$ with $\bar{\Omega} \subset B_{R}$, we set $\Omega_{R}:=B_{R} \backslash \bar{\Omega}$. It will be convenient to fix a real number $S \in(0, \infty)$ with $\bar{\Omega} \subset B_{S}$. For technical reasons, we will not only consider differential equation (1.2), but also its adjoint equation. In order to work on both problems simultaneously, we take $\mu \in\{0,1\}$ and introduce the following boundary value problem:

$$
-\Delta U+\mu \tau \partial_{1} U+\lambda U+\nabla \Pi=G, \quad \operatorname{div} U=0 \quad \text { in } \bar{\Omega}^{c}, \quad U \mid \partial \Omega=0 .
$$

In the proof of Theorem 5.1 and 6.4 below, some results preceding these theorems and involving the quantity $\mu$ are exploited both in the case $\mu=1$ and $\mu=-1$. Therefore the parameter $\mu$ is not fixed. Instead, wherever it is involved, it should be considered as a variable with range $\{-1,1\}$. But this will not be stated explicitly in what follows.

We write $\Re z$ for the real part, $\Im z$ for the imaginary part and $\bar{z}$ for the complex conjugate of a number $z \in \mathbb{C}$. If $A \subset \mathbb{R}^{3}$, let $A^{c}$ denote the complement $\mathbb{R}^{3} \backslash A$ of $A$ in $\mathbb{R}^{3}$. The 
symbol || designates the Euclidean norm in $\mathbb{R}^{n}$, for any $n \in \mathbb{N}$, the modulus of a complex number, the length $\alpha_{1}+\alpha_{2}+\alpha_{3}$ of a multiindex $\alpha \in \mathbb{N}_{0}^{3}$, as well as the Lebesgue measure of measurable subsets of $\mathbb{R}^{3}$. For $R>0, x \in \mathbb{R}^{3}$, put $B_{R}(x):=\left\{y \in \mathbb{R}^{3}:|x-y|<R\right\}$. If $x=0$, we write $B_{R}$ instead of $B_{R}(0)$. If $A$ is some nonempty set and $\gamma: A \mapsto \mathbb{C}$ a function, we abbreviate $|\gamma|_{\infty}:=\sup \{|\gamma(x)|: x \in A\}$. Let $V: \mathbb{R}^{3} \mapsto \mathbb{C}$ and $W: \mathbb{R}^{3} \mapsto \mathbb{C}$ be measurable functions with $\int_{\mathbb{R}^{3}}|V(x-y)||W(y)| d y<\infty$ for a. e. $x \in \mathbb{R}^{3}$. Then we define $(V * W)(x):=\int_{\mathbb{R}^{3}} V(x-y) W(y) d y$ for a. e. $x \in \mathbb{R}^{3}$.

For $p \in[1, \infty], A \subset \mathbb{R}^{3}$ measurable, we write $L^{p}(A)$ for the usual Lebesgue space on $A$ associated with $p$, and \|\|$_{p}$ for the usual norm of this space. Let $A \subset \mathbb{R}^{3}$ be open, $p \in[1, \infty)$ and $m \in \mathbb{N}$. Then the symbol $W^{m, p}(A)$ stands for the usual Sobolev of order $m$ and exponent $p$. The standard norm of this space is designated by \|\|$_{m, p}$. The space $W_{0}^{m, p}(A)$ is defined as the closure of $C_{0}^{\infty}(A)$ with respect to the norm of $W^{m, p}(A)$, and is equipped with this norm. For any open set $B \subset \mathbb{R}^{3}$, and for $p$ and $m$ as before, the spaces $L_{l o c}^{p}(B), W_{l o c}^{m, p}(B)$ are to contain those functions $v$ from $B$ into $\mathbb{C}$ that satisfy the relations $v \mid A \in L^{p}(A)$ and $v \mid A \in W^{m, p}(A)$, respectively, for any open and bounded set $A \subset \mathbb{R}^{3}$ with $\bar{A} \subset B$. If $A \subset \mathbb{R}^{3}$ is an open set with bounded $C^{2}$-boundary $\partial A$, then the Sobolev space $W^{r, p}(\partial A)$ with $p \in(1, \infty), r \in(1,2)$ is to be defined as in [16, section 6.8.6].

Let $n \in \mathbb{N}, A$ a non-empty set and $\mathcal{V}$ a vector space consisting of functions from $A$ into $\mathbb{C}$. Suppose a norm denoted by \|\| has been introduced on this space. Then we set $\left\|\left(f_{1}, \ldots, f_{n}\right)\right\|^{(n)}:=\left(\sum_{j=1}^{n}\left\|f_{j}\right\|^{2}\right)^{1 / 2}$ for $\left(f_{1}, \ldots, f_{n}\right) \in \mathcal{V}^{n}$. The mapping \|\|$^{(n)}$ is a norm on $\mathcal{V}^{n}$, and we equip $\mathcal{V}^{n}$ with this norm. However, we will write \|\| for this norm too, instead of \|\|$^{(n)}$.

If $A \subset \mathbb{R}^{3}$ is open and $p \in(1, \infty)$, define $H_{p}(A)$ as the closure of the set $\left\{\phi \in C_{0}^{\infty}(A)^{3}\right.$ : $\operatorname{div} \phi=0\}$ with respect to the norm \|\|$_{p}$ of $L^{p}(A)^{3}$. If in addition $n \in \mathbb{N}$, we write $W_{0}^{-1, p}(A)^{n}$ for the space dual to $W_{0}^{1, p^{\prime}}(A)^{n}$. The norm \|\|$_{-1, p}$ of $W_{0}^{-1, p}(A)^{n}$ is to be understood as the operator norm of this space, that is, $\|\mathfrak{F}\|_{-1, p}:=\sup \left\{|F(V)| /\|V\|_{1, p^{\prime}}\right.$ : $\left.V \in W_{0}^{1, p^{\prime}}(A)^{n}, V \neq 0\right\}$ for any linear bounded functional $\mathfrak{F}$ on $W_{0}^{1, p^{\prime}}(A)^{n}$. We define

$$
\mathcal{D}_{0}^{1,2}\left(\bar{\Omega}^{c}\right):=\left\{V \in W_{l o c}^{1,2}\left(\bar{\Omega}^{c}\right): \nabla V \in L^{2}\left(\bar{\Omega}^{c}\right)^{9}, V \in L^{6}(V) \text { and } V \mid \partial \Omega=0\right\} .
$$

The mapping $V \mapsto\|\nabla V\|_{2}, V \in \mathcal{D}_{0}^{1,2}\left(\bar{\Omega}^{c}\right)$, is a norm (Theorem 2.5); we equip the space $\mathcal{D}_{0}^{1,2}\left(\bar{\Omega}^{c}\right)$ with this norm. The Cantor completion of $C_{0}^{\infty}\left(\bar{\Omega}^{c}\right)$ with respect to the norm $V \mapsto\|\nabla V\|_{2}$ on $C_{0}^{\infty}\left(\bar{\Omega}^{c}\right)$ is isomorphic to $\mathcal{D}_{0}^{1,2}\left(\bar{\Omega}^{c}\right)([17$, p. 83-84, 105-106]). However, in our context it is not practical to work with this completion. The space dual to $\mathcal{D}_{0}^{1,2}\left(\bar{\Omega}^{c}\right)^{3}$ is denoted by $\mathcal{D}_{0}^{-1,2}\left(\bar{\Omega}^{c}\right)^{3}$. It is equipped with its natural norm denoted by \|\|$_{-1,2}$ and defined by

$$
\|\mathfrak{F}\|_{-1,2}:=\sup \left\{|F(\gamma)| /\|\nabla \gamma\|_{2}: \gamma \in \mathcal{D}_{0}^{1,2}\left(\bar{\Omega}^{c}\right)^{3}, \gamma \neq 0\right\},
$$

for any linear and bounded functional $\mathfrak{F}$ from $\mathcal{D}_{0}^{1,2}\left(\bar{\Omega}^{c}\right)^{3}$ into $\mathbb{C}$. We refer to Theorem 2.6 for more properties of this space. In this theorem, it is explained in which sense functions with domain $\bar{\Omega}^{c}$ may belong to $\mathcal{D}_{0}^{-1,2}\left(\bar{\Omega}^{c}\right)^{3}$. For functions $V \in \mathcal{D}_{0}^{-1,2}\left(\bar{\Omega}^{c}\right)^{3} \cap L^{2}\left(\bar{\Omega}^{c}\right)^{3}$, we use the notation $\|V\|_{*}:=\|V\|_{-1,2}+\|V\|_{2}$. If $p \in(1, \infty), n \in\{1,3\}$ and $G \in L_{l o c}^{1}\left(\mathbb{R}^{3}\right)^{n}$, we define

$$
\|G\|_{-1, p, \mathbb{R}^{3}}:=\sup \left\{\int_{\mathbb{R}^{3}} G \cdot \gamma d x /\|\nabla \gamma\|_{p^{\prime}}: \gamma \in C_{0}^{\infty}\left(\mathbb{R}^{3}\right)^{n}, \gamma \neq 0\right\} .
$$


We further put $\widetilde{\mathcal{D}}_{0}^{-1, p}\left(\mathbb{R}^{3}\right)^{n}:=\left\{G \in L_{\text {loc }}^{1}\left(\mathbb{R}^{3}\right)^{n}:\|G\|_{-1, p, \mathbb{R}^{3}}<\infty\right\}$. The preceding two notation should be considered as abbreviations. We will not use any functional analytic properties of either the mapping \|\|$_{-1, p, \mathbb{R}^{3}}$ or the space $\widetilde{\mathcal{D}}_{0}^{-1, p}\left(\mathbb{R}^{3}\right)^{n}$. For convenience, we will write \|\|$_{-1, p}$ instead of \|\|$_{-1, p, \mathbb{R}^{3}}$. Care has to be taken as concerns the quantity $\|\phi\|_{-1,2}$ if $\phi \in C_{0}^{\infty}\left(\bar{\Omega}^{c}\right)^{3}$. In fact, for any open set $A \subset \mathbb{R}^{3}$, we consider functions from $C_{0}^{\infty}(A)$ as functions with domain $\mathbb{R}^{3}$ (but, of course, with compact support in $A$ ). So, if $\phi \in C_{0}^{\infty}\left(\bar{\Omega}^{c}\right)^{3}$, we have to distinguish between $\|\phi\|_{-1,2}$ and $\left\|\phi \mid \bar{\Omega}^{c}\right\|_{-1,2}$, with the first expression denoting $\|\phi\|_{-1,2, \mathbb{R}^{3}}$, and the second involving the norm \|\|$_{-1,2}$ of $\mathcal{D}_{0}^{-1,2}\left(\bar{\Omega}^{c}\right)^{3}$; see Theorem 2.6 in this respect.

The symbol $C$ is to denote numerical constants, and $C\left(\gamma_{1}, \ldots, \gamma_{n}\right)$ constants depending exclusively on $\gamma_{1}, \ldots, \gamma_{n} \in(0, \infty)$, for some $n \in \mathbb{N}$. However, such precise indications on how a constant depends on parameters will be given only at some places. In order to avoid that our presentation becomes too unwieldy, most of the time we take the point of view that the dependence of our constants on parameters should be clear from context. In that situation the symbol $\mathfrak{C}$ is used to denote generic constants. Often they depend on $\tau$ and on $\Omega$, in particular on the parameter $S$ introduced above. The notation $\mathfrak{C}\left(\gamma_{1}, \ldots, \gamma_{n}\right)$ means that we want to stress the dependence of the constant in question on $\gamma_{1}, \ldots, \gamma_{n} \in(0, \infty)$, for some $n \in \mathbb{N}$. But a constant denoted in this way may depend on other quantities as well.

We define $N(x):=-(4 \pi|x|)^{-1}$ for $x \in \mathbb{R}^{3} \backslash\{0\}$ (fundamental solution of the Poisson equation), and $E^{(\lambda)}(x):=(4 \pi|x|)^{-1} e^{-\left(\lambda+(\tau / 2)^{2}\right)^{1 / 2}|x|+\tau x_{1} / 2}$ for $x \in \mathbb{R}^{3} \backslash\{0\}, \lambda \in \mathbb{C}$ with $\Re \lambda \geq 0$ (fundamental solution of the scalar Oseen equation $-\Delta V+\tau \partial_{1} V=G$ if $\lambda=0$, and of the associated resolvent equation if $\lambda \neq 0$ ).

\section{Various auxiliary results.}

$\langle$ theoremT3.10〉

We start by indicating some properties of Sobolev spaces.

Theorem 2.1 ([17, Lemma II.6.1]) Let $p \in(1, \infty), A \subset \mathbb{R}^{3}$ open, bounded, with Lipschitz boundary. Let $B \in\left\{A, \bar{A}^{c}, \mathbb{R}^{3}\right\}$, and $V \in W_{\text {loc }}^{1,1}(B)$ with $\nabla V \in L^{p}(B)^{3}$. Then $V \in W^{1, p}(A)$ in the case $B=A, V \in W_{\text {loc }}^{1, p}\left(\mathbb{R}^{3}\right)$ in the case $B=\mathbb{R}^{3}$, and $V \mid \bar{A}^{c} \cap B_{R} \in$ $W^{1, p}\left(\bar{A}^{c} \cap B_{R}\right)$ for any $R \in(0, \infty)$ with $\vec{A} \subset B_{R}$ if $B=\bar{A}^{c}$.

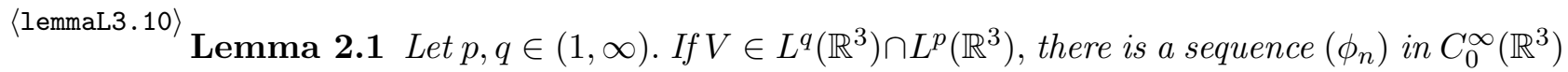
with $\left\|V-\phi_{n}\right\|_{s} \rightarrow 0$ for $s \in\{p, q\}$.

Let $V \in W_{\text {loc }}^{1,1}\left(\mathbb{R}^{3}\right) \cap L^{p}\left(\mathbb{R}^{3}\right)$ with $\nabla V \in L^{q}\left(\mathbb{R}^{3}\right)^{3}$. Then there is a sequence $\left(\psi_{n}\right)$ in $C_{0}^{\infty}\left(\mathbb{R}^{3}\right)$ such that $\left\|V-\psi_{n}\right\|_{p} \rightarrow 0$ and $\left\|\nabla V-\nabla \psi_{n}\right\|_{q} \rightarrow 0$

$\langle$ theoremT3.20

Proof: Use Friedrich's mollifier.

Theorem 2.2 ([17, Theorem III.4.2]) Let $p \in(1, \infty)$ and $V \in W_{0}^{1, p}\left(\bar{\Omega}^{c}\right)^{3}$ with div $V=$ 0 . Then there is a sequence $\left(\phi_{n}\right)$ in $C_{0}^{\infty}\left(\bar{\Omega}^{c}\right)^{3}$ with div $\phi_{n}=0$ for $n \in \mathbb{N}$ and $\left\|\phi_{n}-V\right\|_{1, p} \rightarrow$ 0 .

It is perhaps not so well known that $L^{q}$ is compactly imbedded in $W^{-1, q}$ : 
Theorem 2.3 ([17, Theorem II.5.3]) Let $A \subset \mathbb{R}^{3}$ be open and bounded, $q \in(1, \infty)$, and $\left(V_{n}\right)$ a bounded sequence in $L^{q}(A)$. Then there is $V \in L^{q}(A)$ and a subsequence $\left(W_{n}\right)$ of $\left(V_{n}\right)$ such that $\left\|W_{n}-V\right\|_{-1, q} \rightarrow 0$.

The next theorem deals with the decay behaviour of functions $V$ in exterior domains $\bar{A}^{c}$ with $\nabla V \in L^{p}\left(\bar{A}^{c}\right)^{3}$ for some $p \in(1,3)$.

Theorem 2.4 Let $A \subset \mathbb{R}^{3}$ be either an open bounded nonempty set with Lipschitz boundary, or $A=\emptyset$. Let $q \in(1,3)$ and $V \in W_{\text {loc }}^{1,1}\left(\bar{A}^{c}\right)$ with $\nabla V \in L^{q}\left(\bar{A}^{c}\right)^{3}$. Then there is $V^{*} \in \mathbb{R}$ such that $V-V^{*} \in L^{3 q /(3-q)}\left(\bar{A}^{c}\right)$, and the following five assertion are equivalent:

1.) $V \in L^{r}\left(\bar{A}^{c}\right)$ for some $r \in(1, \infty)$; 2.) $V \in L^{3 q /(3-q)}\left(\bar{A}^{c}\right)$; 3.) $\int_{\partial B_{1}}|V(R x)|^{q} d o_{x} \rightarrow$ $0(R \rightarrow \infty)$; 4.) $\int_{\partial B_{1}}|V(R x)| d o_{x} \rightarrow 0(R \rightarrow \infty)$; 5.) $V^{*}=0$.

If one - and therefore everyone - of these assertions holds, the ensuing inequality is valid:

$$
\|V\|_{3 q /(3-q)} \leq \mathfrak{C}(q)\|\nabla V\|_{q} .
$$

Proof: According to [17, Lemma II.6.3], the relation $\nabla V \in L^{q}\left(\bar{A}^{c}\right)^{3}$ implies there is $V^{*} \in \mathbb{R}$ with $\int_{\partial B_{1}} \mid\left(V(R x)-\left.V^{*}\right|^{q} d o_{x} \rightarrow 0(R \rightarrow \infty)\right.$. Moreover, by [17, Theorem II.6.1], we have $V-V^{*} \in L^{3 q /(3-q)}\left(\bar{A}^{c}\right)$. Starting from these relations, we showed elsewhere ([8, Lemma 2.4], [10, Lemma 2.1]) that assertion 2.) follows from 1.). Suppose that assertion 3.) holds. Then we get from the convergence relation at the beginning of this proof that $V^{*}=0$, so assertion 2.) holds because $V-V^{*} \in L^{3 q /(3-q)}\left(\bar{A}^{c}\right)$. If assertion 2.) is valid, we may deduce from the preceding relation that $V^{*}=0$, so 3.) follows from the convergence result at the beginning of this proof. Suppose that assertion 4.) holds. Then we may choose a sequence $\left(R_{n}\right)$ in $(0, \infty)$ such that $R_{n} \rightarrow \infty$ and $V\left(R_{n} x\right) \rightarrow 0(n \rightarrow \infty)$ for a. e. $x \in \partial B_{1}$. But again by the convergence result at the beginning of this proof, there is a subsequence $\left(S_{n}\right)$ of $\left(R_{n}\right)$ such that $\left|V\left(S_{n} x\right)-V^{*}\right|^{q} \rightarrow 0$ for a. e. $x \in \partial B_{1}$. Recalling the choice of $\left(R_{n}\right)$, we may conclude that $V^{*}=0$, so assertion 3.) follows by referring once more to the beginning of this proof. Obviously 3.) implies 4.). Inequality (2.1) holds according to [17, Theorem II.6.1].

As a consequence of the preceding theorem, we get that $C_{0}^{\infty}\left(\mathbb{R}^{3}\right)^{3} \subset \widetilde{\mathcal{D}}_{0}^{-1, p}\left(\mathbb{R}^{3}\right)^{3}$ if $p>3 / 2$ : corollaryC3.15>

Corollary 2.1 Let $p \in(3 / 2, \infty)$ and $\phi \in C_{0}^{\infty}\left(\mathbb{R}^{3}\right)^{3}$. Then $\phi \in \widetilde{\mathcal{D}}_{0}^{-1, p}\left(\mathbb{R}^{3}\right)^{3}$.

Proof: We have $p^{\prime} \in(1,3)$, so we get with Hölder's inequality and (2.1) that $\left|\int_{\mathbb{R}^{3}} \phi \gamma d x\right| \leq$ $\|\phi\|_{(1 / 3+1 / p)^{-1}}\|\gamma\|_{3 p^{\prime} /\left(3-p^{\prime}\right)} \leq \mathfrak{C}\|\phi\|_{(1 / 3+1 / p)^{-1}}\|\nabla \gamma\|_{p^{\prime}}$ for $\gamma \in C_{0}^{\infty}\left(\mathbb{R}^{3}\right)^{3}$.

In the ensuing lemma, we consider how functions $V$ in exterior domains decay in the $L^{q}$-norm if $\nabla V$ is an $L^{q}$-function and $V$ and $L^{r}$-function, with $q$ not necessarily equal to $r$.

Lemma 2.2 Let $q, r \in(1, \infty), R_{0} \in(0, \infty)$. Then

$$
\begin{aligned}
& R^{-1}\left\|V \mid B_{2 R \backslash R}\right\|_{q} \leq \mathfrak{C}\left(q, r, R_{0}\right)\left(\left\|V\left|B_{R}^{c}\left\|_{r}+\right\| \nabla V\right| B_{R}^{c}\right\|_{q}+R^{-1}\|V\|_{r}+R^{-1}\|\nabla V\|_{q}\right) \\
& \text { for } R \in\left(R_{0}, \infty\right), V \in W_{l o c}^{1,1}\left({\overline{B_{R_{0}}}}^{c}\right) \cap L^{r}\left({\overline{B_{R_{0}}}}^{c}\right) \text { with } \nabla V \in L^{q}\left({\overline{B_{R_{0}}}}^{c}\right)^{3} .
\end{aligned}
$$


Proof: We proceed as in [17, p. 225]. Take $R$ and $V$ as in the lemma. First suppose that $q<3$. Then Hölder's inequality yields $\left\|V\left|B_{2 R} \backslash B_{R}\left\|_{q} \leq \mathfrak{C} R\right\| V\right| B_{R}^{c}\right\|_{3 q /(3-q)}$. On the other hand, a scaling argument and (2.1) with $A=B_{R_{0}}$ imply

$$
\begin{aligned}
& \left\|V\left|B_{R}^{c}\left\|_{3 q /(3-q)}=\left(R / R_{0}\right)^{(3-q) / q}\right\| V\left(\left(R / R_{0}\right) \cdot\right)\right|{\overline{B_{R_{0}}}}^{c}\right\|_{3 q /(3-q)} \\
& \leq C\left(R_{0}, q\right)\left(R / R_{0}\right)^{(3-q) / q}\left\|\nabla\left[V\left(\left(R / R_{0}\right) \cdot\right) \mid{\overline{B_{R_{0}}}}^{c}\right]\right\|_{q}=C\left(R_{0}, q\right)\left\|\nabla V \mid B_{R}^{c}\right\|_{q} .
\end{aligned}
$$

Thus, in the case $q<3$, we have found that $\left\|V\left|B_{2 R} \backslash B_{R}\left\|_{q} \leq C\left(R_{0}, q\right) R\right\| \nabla V\right| B_{R}^{c}\right\|_{q}$. Next suppose that $q \geq 3$ and $q \leq r$. Due to the second assumption, we get by Hölder's inequality that $\left\|V\left|B_{2 R} \backslash B_{R}\left\|_{q} \leq C R^{3(1 / q-1 / r)}\right\| V\right| B_{R}^{c}\right\|_{r}$. But $q \geq 3$ and $R \geq R_{0}$, so we may conclude that $\left\|V\left|B_{2 R} \backslash B_{R}\left\|_{q} \leq C R_{0}^{3(1 / q-1 / r-1 / 3)} R\right\| V\right| B_{R}^{c}\right\|_{r}$.

Finally suppose that $q \geq 3$ and $q>r$. Then $a:=(1 / r-1 / q) /(1 / r-1 / q+1 / 3) \in(0,1)$. Obviously $1 / q=a(1 / q-1 / 3)+(1-a) / r$, so by [17, Lemma II.3.3], we obtain $\|W\|_{q} \leq$ $C(q, r)\|\nabla W\|_{q}^{a}\|W\|_{r}^{1-a} \leq C(q, r)\left(\|\nabla W\|_{q}+\|W\|_{r}\right)$ for $W \in W_{l o c}^{1,1}\left(\mathbb{R}^{3}\right) \cap L^{r}\left(\mathbb{R}^{3}\right)$ with $\nabla W \in L^{q}\left(\mathbb{R}^{3}\right)^{3}$. Now we split $V$ into a sum of a $W_{l o c}^{1,1}$-function on $\mathbb{R}^{3}$ and a $W_{l o c}^{1,1}$-function on a bounded domain. To this end, we set $B:=B_{R_{0}+1} \backslash \overline{B_{R_{0}}}$, and take $\varphi \in C^{\infty}\left(\mathbb{R}^{3}\right)$ with $\varphi \mid B_{R_{0}+3 / 4}^{c}=1$ and $\varphi \mid B_{R_{0}+1 / 4}=0$. Obviously $\varphi V \in W_{l o c}^{1,1}\left(\mathbb{R}^{3}\right) \cap L^{r}\left(\mathbb{R}^{3}\right)$. According to Theorem 2.1, we have $V \mid B \in W^{1, q}(B)$, so $\nabla(\varphi V) \in L^{q}\left(\mathbb{R}^{3}\right)$. Now the above estimate of $\|W\|_{q}$ yields $\|\varphi V\|_{q} \leq \mathfrak{C}\left(q, r, R_{0}\right)\left(\|\nabla V\|_{q}+\|V \mid B\|_{q}+\|V\|_{r}\right)$. Hence

$$
\|V\|_{q} \leq\|\varphi V\|_{q}+\|(1-\varphi) V\|_{q} \leq \mathfrak{C}\left(q, r, R_{0}\right)\left(\|\nabla V\|_{q}+\|V \mid B\|_{q}+\|V\|_{r}\right) .
$$

But the term $\|V \mid B\|_{q}$ my be estimated by Poincaré's inequality for functions with mean value zero. In fact, abbreviate $m:=|B|^{-1} \int_{B} V d x$. Then $\left\|V\left|B\left\|_{q} \leq\right\|(V-m)\right| B\right\|_{q}+$ $|m||B|^{1 / q}$, with $\|(V-m) \mid B\|_{q}$ bounded by $C\left(q, R_{0}\right)\|\nabla V \mid B\|_{q}$, whereas $|m||B|^{1 / q}$ may be estimated by $|B|^{-1+1 / q}\|V \mid B\|_{1}$, and thus by $C\left(q, r, R_{0}\right)\|V\|_{r}$. Therefore from (2.2), $\|V\|_{q} \leq \mathfrak{C}\left(r, R_{0}\right)\left(\|\nabla V\|_{q}+\|V\|_{r}\right)$. The lemma follows from the preceding inequality and from our estimates of $\left\|V \mid B_{2 R} \backslash B_{R}\right\|_{q}$ in the cases $q<3$ and $q \geq 3, q \leq r$, respectively.

Next we collect some properties of $\mathcal{D}_{0}^{1,2}\left(\bar{\Omega}^{c}\right)$ and $\mathcal{D}_{0}^{-1,2}\left(\bar{\Omega}^{c}\right)$.

Theorem 2.5 The mapping $(V, W) \mapsto \int_{\bar{\Omega}^{c}} \nabla V \cdot \nabla W d x$ is a scalar product on $\mathcal{D}_{0}^{1,2}\left(\bar{\Omega}^{c}\right)$, and $\mathcal{D}_{0}^{1,2}\left(\bar{\Omega}^{c}\right)$ equipped with this scalar product is a Hilbert space, with associated norm $V \mapsto\|\nabla V\|_{2}$. Moreover the inequality $\|V\|_{6} \leq \mathfrak{C}\|\nabla V\|_{2}$ holds for $V \in \mathcal{D}_{0}^{1,2}\left(\bar{\Omega}^{c}\right)$, and the set $C_{0}^{\infty}\left(\bar{\Omega}^{c}\right)$ is dense in $\mathcal{D}_{0}^{1,2}\left(\bar{\Omega}^{c}\right)$.

Proof: For the Hilbert space property, we refer to [17, p. 105] or [24, Theorem I.2.2, I.2.8]. The inequality $\|V\|_{6} \leq \mathfrak{C}\|\nabla V\|_{2}$ holds according to (2.1). As concerns density of $C_{0}^{\infty}\left(\bar{\Omega}^{c}\right)$ in $\mathcal{D}_{0}^{1,2}\left(\bar{\Omega}^{c}\right)$, we refer to Theorem 2.4 and [17, Theorem II.7.1], or to [24, Theorem I.2.8].

$\langle$ theoremT3.70〉

Theorem 2.6 Let $g \in L_{\text {loc }}^{1}\left(\bar{\Omega}^{c}\right)^{3}$ and put $G_{g}(\varphi):=\int_{\bar{\Omega}^{c}} g \cdot \varphi d x$ for $\varphi \in C_{0}^{\infty}\left(\bar{\Omega}^{c}\right)^{3}$. Suppose that $\|g\|_{-1,2}^{*}:=\sup \left\{G_{g}(\varphi) /\|\nabla \varphi\|_{2}: \varphi \in C_{0}^{\infty}\left(\bar{\Omega}^{c}\right)^{3}, \varphi \neq 0\right\}<\infty$. Then there is a unique element $F_{g} \in \mathcal{D}_{0}^{-1,2}\left(\bar{\Omega}^{c}\right)^{3}$ with $F_{g} \mid C_{0}^{\infty}\left(\bar{\Omega}^{c}\right)^{3}=G_{g}$, and the relation $\|g\|_{-1,2}^{*}=\left\|F_{g}\right\|_{-1,2}$ holds. In this case, we write $g$ instead of $F_{g}$, and $g \in \mathcal{D}_{0}^{-1,2}\left(\bar{\Omega}^{c}\right)^{3}$ instead of $\|g\|_{-1,2}^{*}<\infty$.

If $g \in L^{6 / 5}\left(\bar{\Omega}^{c}\right)^{3}$, then $g \in \mathcal{D}_{0}^{-1,2}\left(\bar{\Omega}^{c}\right)^{3}, F_{g}(\varphi)=\int_{\bar{\Omega}^{c}} g \cdot \varphi d x$ for any $\varphi \in \mathcal{D}_{0}^{1,2}\left(\bar{\Omega}^{c}\right)^{3}$, and $\|g\|_{-1,2} \leq \mathfrak{C}(\Omega)\|g\|_{6 / 5}$. 
For any $h \in \mathcal{D}_{0}^{-1,2}\left(\bar{\Omega}^{c}\right)^{3}$, there is a sequence $\left(\varphi_{n}\right)$ in $C_{0}^{\infty}\left(\bar{\Omega}^{c}\right)^{3}$ with $\left\|h-\varphi_{n}\right\|_{-1,2} \rightarrow 0$.

Proof: Hahn-Banach's theorem and the definition of $\|g\|_{-1,2}^{*}$ yield existence of $F_{g} \in$ $\mathcal{D}_{0}^{-1,2}\left(\bar{\Omega}^{c}\right)^{3}$ with $F_{g} \mid C_{0}^{\infty}\left(\bar{\Omega}^{c}\right)^{3}=G_{g}$ and $\|g\|_{-1,2}^{*}=\left\|F_{g}\right\|_{-1,2}$. Uniqueness of $F_{g}$ follows from the density of $C_{0}^{\infty}\left(\bar{\Omega}^{c}\right)$ in $\mathcal{D}_{0}^{1,2}\left(\bar{\Omega}^{c}\right)$ (Theorem 2.5). By Hölder's inequality and the estimate $\|\varphi\|_{6} \leq \mathfrak{C}\|\nabla \varphi\|_{2}$ (Theorem 2.5), we get that $\int_{\bar{\Omega}^{c}}|g \cdot \varphi| d x \leq\|g\|_{6 / 5}\|\nabla \varphi\|_{2}$ for $\varphi \in C_{0}^{\infty}\left(\bar{\Omega}^{c}\right)^{3}$, so the claims of the theorem related to the case $g \in L^{6 / 5}\left(\bar{\Omega}^{c}\right)^{3}$ are true. The last statement of the theorem holds according to [17, Theorem II.8.1].

We turn to the boundary value problem $\operatorname{div} V=G$ in $B, V \mid \partial B=0$, for annular domains $B \subset \mathbb{R}^{3}$.

Theorem 2.7 ([4, Theorem 2.4]) Let $R, \widetilde{R} \in(0, \infty)$ with $R<\widetilde{R}$, and put $B:=$ $B_{\widetilde{R}} \backslash \overline{B_{R}}$. Then, for any $q \in(1, \infty)$ and $m \in\{0,1\}$, there is a linear operator $\mathfrak{D}:=$ $\mathfrak{D}(q, m, R, \widetilde{R})$ from $\left\{g \in W_{0}^{m, q}(B): \int_{B} g d x=0\right\}$ into $W_{0}^{m+1, q}(B)^{3}$ such that div $\mathfrak{D}(g)=$ $g$ and $\|\mathfrak{D}(g)\|_{m+1, q} \leq C(q, m, R, \widetilde{R})\|g\|_{m, q}$ for $g \in W_{0}^{m, q}(B)$ with $\int_{B} g d x=0$.

Moreover, for $p, m$ as before, and for $g \in C_{0}^{\infty}(B)$ with $\int_{B} g d x=0$, the function $\mathfrak{D}(g)$ (or more precisely: the zero extension of $\mathfrak{D}(g \mid B)$ to $\left.\mathbb{R}^{3}\right)$ belongs to $C_{0}^{\infty}(B)^{3}$.

Finally, if $p, q \in(1, \infty), m, n \in\{0,1\}$ and $g \in C_{0}^{\infty}(B)$ with $\int_{B} g d x=0$, then the functions $\mathfrak{D}(p, m, R, \widetilde{R})(g)$ and $\mathfrak{D}(q, n, R, \widetilde{R})(g)$ coincide.

The ensuing lemma deals with solutions to the boundary value problem $\operatorname{div} V=G$ in $B, V \mid \partial B=0$, with $B$ belonging to the set $\left\{B_{2 n} \backslash B_{n}:, n \in \mathbb{N}\right\}$ of annular domains. The lemma indicates how the $L^{p}$-norm of these solutions depends on $n$.

$\langle$ lemmaL3.50〉

Lemma 2.3 Let $q \in(1, \infty), \nu \in\{0,1\}, C_{0}>0, \mathcal{W}_{n}:=\left\{g \in W_{0}^{\nu, q}\left(B_{2 n} \backslash \overline{B_{n}}\right)\right.$ : $\left.\int_{B_{2 n} \backslash \overline{B_{n}}} g d x=0\right\}$ for $n \in \mathbb{N}, \mathcal{D}: \mathcal{W}_{1} \mapsto W_{0}^{\nu+1, q}\left(B_{2} \backslash \overline{B_{1}}\right)^{3}$ a mapping such that $\operatorname{div} \mathcal{D}(g)=$ $g$ and $\|\mathcal{D}(g)\|_{1+\nu, q} \leq C_{0}\|g\|_{\nu, q}$ for $g \in \mathcal{W}_{1}$. For $n \in \mathbb{N}, g \in \mathcal{W}_{n}, x \in B_{2 n} \backslash \overline{B_{n}}$, set $\mathcal{D}_{n}(g)(x):=n \mathcal{D}\left(g \circ\left(n i d_{B_{2} \backslash \overline{B_{1}}}\right)\right)((1 / n) x)$, with $i d_{B_{2} \backslash \overline{B_{1}}}$ the identity function on $B_{2} \backslash \overline{B_{1}}$.

Then, for $n \in \mathbb{N}, g \in \mathcal{W}_{n}$, we have $\mathcal{D}_{n}(g) \in W_{0}^{\nu+1, q}\left(B_{2 n} \backslash \overline{B_{n}}\right)^{3}$, $\operatorname{div} \mathcal{D}_{n}(g)=g$ and $\left\|\partial^{\alpha} \mathcal{D}_{n}(g)\right\|_{q} \leq C_{0} n^{1-|\alpha|}\|g\|_{q}$ for $\alpha \in \mathbb{N}_{0}^{3}$ with $|\alpha| \leq 1$ in the case $\nu=0$, as well as $\left\|\partial_{l} \partial_{m} \mathcal{D}_{n}(g)\right\|_{q} \leq C_{0}\|g\|_{1, q}$ for $l, m \in\{1,2,3\}$ in the case $\nu=1$.

Proof: Direct calculation, via scaling.

Next we recall some results related to Poisson's equation $\Delta V=G$.

$\langle$ theoremT3.90〉

Theorem 2.8 (Weyl's lemma) Let $A \subset \mathbb{R}^{3}$ be open and $V \in L_{\text {loc }}^{1}(A)$ such that the integral $\int_{A} V \Delta \varphi d x$ vanishes for any $\varphi \in C_{0}^{\infty}(A)$. Then $V \in C^{\infty}(A)$ and $\Delta V=0$.

Proof: An elementary proof may be found in [24, Appendix].

The consequence of Theorem 2.8 we have in mind is the ensuing corollary, which in principle is well known, but which we indicate because we do not know a direct reference.

Corollary 2.2 Let $A \subset \mathbb{R}^{3}$ be open and connected, $\Pi \in W_{\text {loc }}^{1,1}(A)$ with $\nabla \Pi=0$. Then there is $c \in \mathbb{R}$ with $\Pi(x)=c$ for a. e. $x \in A$.

Proof: Theorem 2.8 yields $\Pi \in C_{0}^{\infty}(A)$. 
The following theorem deals with the Newton potential. Since some subtleties of this potential play an important role in what follows, we state them here. Concerning proofs, they are, of course, well known. We only mention for completeness that the relations in (2.3) follow by integration by parts and Lebesgue's theorem, the estimates in (2.5) and (2.6) are a consequence of Hardy-Littlewood-Sobolev's inequality, whereas inequality (2.4) may deduced from Calderon-Zygmund's inequality. The other claims of the theorem follow from these inequalities via density arguments. Lemma 2.1 is useful in this context.

$\left\langle\right.$ theoremT3.100〉 Theorem 2.9 Let $\phi \in C_{0}^{\infty}\left(\mathbb{R}^{3}\right)$. Then the integral $\int_{\mathbb{R}^{3}}\left|\partial^{\alpha} N(x-y) \partial^{\beta} \phi(y)\right| d y$ is finite for $x \in \mathbb{R}^{3}, \alpha \in \mathbb{N}_{0}^{3}$ with $|\alpha| \leq 1, \beta \in \mathbb{N}_{0}^{3}$, and

$$
\begin{aligned}
& N * \phi \in C^{\infty}\left(\mathbb{R}^{3}\right), \quad \partial^{\beta}(N * \phi)=N * \partial^{\beta}(\phi) \text { for } \beta \in \mathbb{N}_{0}^{3}, \\
& \partial_{l}(N * \phi)=\left(\partial_{l} N\right) * \phi \text { for } 1 \leq l \leq 3, \quad \Delta(N * \phi)=\phi, \\
& \left\|\partial_{l} \partial_{m}(N * \phi)\right\|_{q} \leq C(q)\|\phi\|_{q} \text { for } 1 \leq l, m \leq 3, \quad q \in(1, \infty) .
\end{aligned}
$$

Let $q \in(1,3 / 2), \phi \in L^{q}\left(\mathbb{R}^{3}\right)$. Then

$$
\|N *|\phi|\|_{3 q /(3-2 q)} \leq C(q)\|\phi\|_{q},
$$

in particular $N * \phi \in L^{3 q /(3-2 q)}\left(\mathbb{R}^{3}\right)$ and $\int_{\mathbb{R}^{3}}|N(x-y) \phi(y)| d y<\infty$ for a. e. $x \in \mathbb{R}^{3}$. Moreover $N * \phi \in W_{l o c}^{2,1}\left(\mathbb{R}^{3}\right)$.

Let $q \in(1,3), \phi \in L^{q}\left(\mathbb{R}^{3}\right)$. Then

$$
\left\|\left|\partial_{l} N\right| *|\phi|\right\|_{3 q /(3-q)} \leq C(q)\|\Phi\|_{q} \text { for } 1 \leq l \leq 3,
$$

in particular $\partial_{l} N * \phi \in L^{3 q /(3-q)}\left(\mathbb{R}^{3}\right)$ and $\int_{\mathbb{R}^{3}}\left|\partial_{l} N(x-y)\right||\phi(y)| d y<\infty$ for a. e. $x \in \mathbb{R}^{3}$. Moreover $\left(\partial_{l} N\right) * \phi \in W_{l o c}^{1,1}\left(\mathbb{R}^{3}\right)$ and $\operatorname{div}\left(\left(\partial_{l} N\right) * \phi\right)_{1 \leq l \leq 3}=\phi$.

If $q \in(1,3 / 2), \phi \in L^{q}\left(\mathbb{R}^{3}\right)$, then $\partial_{l}(N * \phi)=\left(\partial_{l} N\right) * \phi(1 \leq l \leq 3)$. If $q \in(1,3)$, $p \in$ $(1, \infty), \phi \in L^{q}\left(\mathbb{R}^{3}\right) \cap L^{p}\left(\mathbb{R}^{3}\right)$, then

$$
\left\|\partial_{m}\left(\left(\partial_{l} N\right) * \phi\right)\right\|_{p} \leq C(p)\|\phi\|_{p} \quad(1 \leq l, m \leq 3) .
$$

In addition $\partial_{l}(N * \phi) \in L^{r}\left(\mathbb{R}^{3}\right)$ for any $r \in(3 / 2, \infty)$ if $\phi \in L^{q}\left(\mathbb{R}^{3}\right)$ for any $q \in(1,3)$, and $\partial_{m} \partial_{l}(N * \phi) \in L^{r}\left(\mathbb{R}^{3}\right)$ for any $r \in(1, \infty)$ if $\phi \in L^{q}\left(\mathbb{R}^{3}\right)$ for any $q \in(1, \infty)$.

Corollary 2.3 Let $p \in(1, \infty), \phi \in \cup_{q \in(1,3)} L^{q}\left(\mathbb{R}^{3}\right) \cap \widetilde{\mathcal{D}}_{0}^{-1, p}\left(\mathbb{R}^{3}\right), \quad l \in\{1,2,3\}$. Then $\left\|\left(\partial_{l} N\right) * \phi\right\|_{p} \leq C(p)\|\phi\|_{-1, p}$.

Proof: We simplify the argument from [17, p. 457-458]. By the assumptions on $\phi$, we may choose $q \in(1,3)$ with $\phi \in L^{q}\left(\mathbb{R}^{3}\right)$, so $\left(\partial_{l} N\right) * \phi \in L^{3 q /(3-q)}\left(\mathbb{R}^{3}\right)$ (Theorem 2.9). Let $\psi \in C_{0}^{\infty}\left(\mathbb{R}^{3}\right)$. In view of $(2.6)$, we may apply Fubini's theorem, to obtain $\int_{\mathbb{R}^{3}}\left(\left(\partial_{l} N\right) *\right.$ $\phi) \psi d x=-\int_{\mathbb{R}^{3}}\left(\left(\partial_{l} N\right) * \psi\right) \phi d x$. Since $q<3$, we have $q^{\prime}>3 / 2$, so $\left(\partial_{l} N\right) * \psi \in L^{q^{\prime}}\left(\mathbb{R}^{3}\right)$ and also $\nabla\left(\left(\partial_{l} N\right) * \psi\right) \in L^{p^{\prime}}\left(\mathbb{R}^{3}\right)^{3}$ by $(2.3)$ and the last two assertions of Theorem 2.9. Thus, by Lemma 2.1 , we may choose a sequence $\left(\gamma_{n}\right)$ in $C_{0}^{\infty}\left(\mathbb{R}^{3}\right)$ such that $\left\|\left(\partial_{l} N\right) * \psi-\gamma_{n}\right\|_{q^{\prime}} \rightarrow 0$ and $\left\|\nabla\left(\left(\partial_{l} N\right) * \psi\right)-\nabla \gamma_{n}\right\|_{p^{\prime}} \rightarrow 0$. We now find

$$
\begin{aligned}
& \left|\int_{\mathbb{R}^{3}}\left(\left(\partial_{l} N\right) * \phi\right) \psi d x\right| \leq \limsup _{n \rightarrow \infty}\left|\int_{\mathbb{R}^{3}} \gamma_{n} \phi d x\right| \leq \limsup _{n \rightarrow \infty}\|\phi\|_{-1, p}\left\|\nabla \gamma_{n}\right\|_{p^{\prime}} \\
& \leq\|\phi\|_{-1, p}\left\|\nabla\left(\left(\partial_{l} N\right) * \psi\right)\right\|_{p^{\prime}} \leq\|\phi\|_{-1, p}\|\| \psi \|_{p^{\prime}},
\end{aligned}
$$


where the last inequality follows from (2.7).

In the rest of this section, we deal with the Helmholtz projection, starting with the whole space case. The special feature of that case is that the projection in question applied to a smooth function is given in an explicit way.

$\langle$ theoremT3.150〉

Theorem 2.10 Let $q \in(1, \infty)$. Then there are linear operators $P_{q}: L^{q}\left(\mathbb{R}^{3}\right)^{3} \mapsto H_{q}\left(\mathbb{R}^{3}\right)$ and $Q_{q}: L^{q}\left(\mathbb{R}^{3}\right) \mapsto W_{\text {loc }}^{1, q}\left(\mathbb{R}^{3}\right)$ such that $P_{q}^{2}=P_{q}, G=P_{q}(G)+\nabla Q_{q}(G),\left\|P_{q}(G)\right\|_{q}+$ $\left\|\nabla Q_{q}(G)\right\|_{q} \leq C(q)\|G\|_{q}$ (in particular $\nabla Q_{q}(G) \in L^{q}\left(\mathbb{R}^{3}\right)^{3}$ and $P_{q}\left(\nabla Q_{q}(G)\right)=0$ ) for $G \in L^{q}\left(\mathbb{R}^{3}\right)^{3}$, and $Q_{q}(G)=N * \operatorname{div} G, P_{q}(G)=G-\left(\left(\partial_{l} N\right) * \operatorname{div} G\right)_{1 \leq l \leq 3}$ for $G \in C_{0}^{\infty}\left(\mathbb{R}^{3}\right)^{3}$.

Proof: See [17, Section III.1, in particular p. 147-148 and Theorem III.1.2].

Corollary 2.4 Let $p, q \in(1, \infty)$ and $G \in L^{p}\left(\mathbb{R}^{3}\right)^{3} \cap L^{q}\left(\mathbb{R}^{3}\right)^{3}$. Then $P_{p}(G)=P_{q}(G)$.

Proof: See [11, Theorem 5] or use Lemma 2.1 and Theorem 2.10.

In the ensuing Theorem 2.11, we introduce the Helmholtz projection on $L^{p}(\bar{\Omega})^{3}$, denoted by $\mathcal{P}_{p}$, and an operator $\mathcal{G}_{p}$ corresponding to the complement of $H_{p}\left(\bar{\Omega}^{c}\right)$ in $L^{p}\left(\bar{\Omega}^{c}\right)^{3}$. Some additional facts that may be deduced from Theorem 2.11 are presented in Corollary 2.5.

$\langle$ theoremT2.10〉

Theorem 2.11 Let $\mathcal{P}_{2}: L^{2}\left(\bar{\Omega}^{c}\right)^{3} \mapsto H_{2}\left(\bar{\Omega}^{c}\right)$ denote the usual projection operator onto the closed subspace $H_{2}(\bar{\Omega})$ of $L^{2}\left(\bar{\Omega}^{c}\right)^{3}$. Let $p \in(1, \infty)$. Then there are linear operators $\mathcal{P}_{p}$ : $L^{p}\left(\bar{\Omega}^{c}\right)^{3} \mapsto H_{p}\left(\bar{\Omega}^{c}\right), \mathcal{G}_{p}: L^{p}\left(\bar{\Omega}^{c}\right)^{3} \mapsto W_{\text {loc }}^{1, p}\left(\bar{\Omega}^{c}\right)$ such that $\mathcal{P}_{p}^{2}=\mathcal{P}_{p}, V=\mathcal{P}_{p}(V)+\mathcal{G}_{p}(V)$,

$$
\left\|\mathcal{P}_{p}(V)\right\|_{p}+\|\nabla \mathcal{G}(V)\|_{p} \leq \mathfrak{C}(p)\|V\|_{p},
$$

in particular $\nabla \mathcal{G}_{p}(V) \in L^{p}\left(\bar{\Omega}^{c}\right)^{3}$ and $\mathcal{P}_{p}\left(\nabla \mathcal{G}_{p}(V)\right)=0$, for $V \in L^{p}\left(\bar{\Omega}^{c}\right)^{3}$, and such that $\mathcal{P}_{p}(V)=\mathcal{P}_{2}(V)$ for $V \in C_{0}^{\infty}\left(\bar{\Omega}^{c}\right)^{3}$.

Proof: See [17, Section III.1, in particular p. 149-152], [15], [21] or [23].

Corollary 2.5 Let $p, q \in(1, \infty)$. Then $\mathcal{P}_{p}(G)=\mathcal{P}_{q}(G)$ for $G \in L^{p}\left(\bar{\Omega}^{c}\right)^{3} \cap L^{q}\left(\bar{\Omega}^{c}\right)^{3}$. Moreover $\left(\mathcal{P}_{p}\right)^{\prime}=\mathcal{P}_{p^{\prime}}$ and $\mathcal{P}_{q}(\nabla \Pi)=0$ for $\Pi \in W_{\text {loc }}^{1, q}\left(\bar{\Omega}^{c}\right)$ with $\nabla \Pi \in L^{q}\left(\bar{\Omega}^{c}\right)^{3}$. In addition, $\mathcal{P}_{p}(G)=G$ for $G \in H_{p}\left(\bar{\Omega}^{c}\right)$.

Proof: The first claim of the corollary follows from Lemma 2.1, (2.8) and the last equation in Theorem 2.11. Concerning the proof of the second statement, let $G \in L^{p}\left(\bar{\Omega}^{c}\right)^{3}, H \in$ $L^{p^{\prime}}\left(\bar{\Omega}^{c}\right)^{3}$. We may choose a sequence $\left(\phi_{n}\right)$ in $C_{0}^{\infty}\left(\bar{\Omega}^{c}\right)^{3}$ with $\operatorname{div} \phi_{n}=0$ for $n \in \mathbb{N}$ and $\left\|\phi_{n}-\mathcal{P}_{p^{\prime}}(H)\right\|_{p^{\prime}} \rightarrow 0$. Then, since $\nabla \mathcal{G}_{p}(G) \in L^{p}\left(\bar{\Omega}^{c}\right)^{3}$ (Theorem 2.11), we have $\int_{\bar{\Omega}^{c}} \nabla \mathcal{G}_{p}(G)$. $\mathcal{P}_{p^{\prime}}(H) d x=\lim _{n \rightarrow \infty} \iint_{\bar{\Omega}^{c}} \nabla \mathcal{G}_{p}(G) \cdot \phi_{n} d x=0$, so $\int_{\bar{\Omega}^{c}} G \cdot \mathcal{P}_{p^{\prime}}(H) d x=\int_{\bar{\Omega}^{c}} \mathcal{P}_{p}(G) \cdot \mathcal{P}_{p^{\prime}}(H) d x$ by Theorem 2.11. An analogous equation holds with $\mathcal{P}_{p}(G) \cdot H$ in the place of $G \cdot \mathcal{P}_{p^{\prime}}(H)$ on the left-hand side. The second equation of the corollary follows. The same type of argument yields that $\int_{\bar{\Omega}^{c}} V \cdot \nabla \Pi d x=0$ for $V \in H_{q^{\prime}}\left(\bar{\Omega}^{c}\right)$ and for $\Pi$ as in the corollary. This equation and the second statement of the corollary yield the third. Finally the preceding equation, the second equation the corollary and Theorem 2.11 imply $\int_{\bar{\Omega}^{c}}(G-$ $\left.\mathcal{P}_{p}(G)\right) \cdot W d x=0$ for $G \in H_{p}\left(\bar{\Omega}^{c}\right)^{3}, W \in L^{p^{\prime}}\left(\bar{\Omega}^{c}\right)^{3}$. This proves the last claim of the corollary. 


\section{The Stokes and Oseen system and associated resolvent problems: some known results.}

We observe that for any weak solution of the Oseen or the Oseen resolvent system, there exists an associated pressure.

$\langle$ theoremT4.20〉

Theorem 3.1 Let $A \subset \mathbb{R}^{3}$ be open, $q \in(1, \infty), \lambda \in \mathbb{C}, G \in L_{\text {loc }}^{q}(A), V \in W_{\text {loc }}^{1,1}(A)^{3}$ with $\nabla V \in L_{l o c}^{q}(A)^{9}, \operatorname{div} V=0$,

$$
\int_{A}\left(\nabla V \cdot \nabla \psi+\left(\mu \tau \partial_{1} V+\lambda V-G\right) \cdot \psi\right) d x=0 \quad \text { for } \psi \in C_{0}^{\infty}(A)^{3} \text { with } \operatorname{div} \psi=0 . \text { (3.1) T4.20.10 }
$$

Then there is $\Pi \in L_{l o c}^{q}(A)$ ("pressure associated with $V$ ") such that

$$
\left.\int_{A}\left(\nabla V \cdot \nabla \psi+\left(\mu \tau \partial_{1} V+\lambda V-G\right) \cdot \psi\right)-\Pi \operatorname{div} \psi\right) d x=0 \quad \text { for } \psi \in C_{0}^{\infty}(A)^{3}
$$

Proof: Theorem 2.1 yields $V \mid B_{\epsilon} \in L^{q}\left(B_{\epsilon}(x)\right)^{3}$ for any $x \in A, \epsilon>0$ with $\overline{B_{\epsilon}(x)} \subset A$. Thus $V \in L_{l o c}^{q}(A)^{3}$, and we may conclude that $-\mu \tau \partial_{1} V-\lambda V+G \in L_{l o c}^{q}(A)^{3}$. Now Theorem 3.1 follows from [17, Lemma IV.1.1] and (3.1).

We cite a theorem on interior regularity of solutions to the Stokes system.

$\langle$ theoremT4.30〉

Theorem 3.2 ([17, Theorem IV.4.1]) Let $A \subset \mathbb{R}^{3}$ be open, $m \in \mathbb{N}_{0}, r \in(1, \infty), G \in$ $W_{l o c}^{m, r}(A)^{3}, V \in W_{l o c}^{1,1}(A)^{3}$ with $\nabla V \in L_{l o c}^{r}(A)^{9}$, div $V=0$ and

$$
\int_{A}(\nabla V \cdot \nabla \psi-G \cdot \psi) d x=0 \quad \text { for } \psi \in C_{0}^{\infty}(A)^{3} \text { with } \operatorname{div} \psi=0
$$

( $V$ weak solution of the Stokes system). Then $V \in W_{l o c}^{2+m, r}(A)^{3}$. Let $\Pi \in L_{l o c}^{r}(A)$ with

$$
\int_{A}(\nabla V \cdot \nabla \psi-\Pi \operatorname{div} \psi-G \cdot \psi) d x=0 \quad \text { for } \psi \in C_{0}^{\infty}(A)^{3}
$$

("pressure associated with $V ")$. Then $\Pi \in W_{l o c}^{1+m, r}(A)$ and $-\Delta V+\nabla \Pi=G$.

The preceding theorem implies interior regularity for solutions of the Oseen resolvent problem (if $\lambda=0$ : Oseen system). For the convenience of the reader, we indicate a proof.

corollaryc4.10) Corollary 3.1 Let $A \subset \mathbb{R}^{3}$ be open, $q, s \in(1, \infty), \lambda \in \mathbb{C}, G \in L_{\text {loc }}^{q}(A)^{3}, V \in W_{\text {loc }}^{1,1}(A)^{3}$ with $\nabla V \in L_{\text {loc }}^{s}(A)^{9}$ and div $V=0$. Suppose that (3.1) holds. Then $V \in W_{l o c}^{2, q}(A)^{3}$. Let $\Pi \in L_{\text {loc }}^{s}(A)$ be a pressure associated with $V$ (Theorem 3.1). Then $\Pi \in W_{l o c}^{1, q}(A)$ and

$$
-\Delta V+\mu \tau \partial_{1} V+\lambda V+\nabla \Pi=G, \quad \operatorname{div} V=0 .
$$

Proof: Theorem 2.1 yields $V \in W_{l o c}^{1, s}(A)^{3}$. Abbreviate $H:=-\mu \tau \partial_{1} V-\lambda V+G$. Put $r_{1}:=\min \{q, s\}$. Then $H$ and $\nabla V$ are $L_{l o c}^{r_{1}}$-functions in $A$, so Theorem 3.2 implies that $V \in W_{l o c}^{2, r_{1}}(A)^{3}, \Pi \in W_{l o c}^{1, r_{1}}(A)$ and (3.5) holds. If $q \leq s$, Corollary 3.1 is proved. Else we apply a Sobolev inequality to obtain $V \in W_{l o c}^{1,3 / 2}(A)^{3}$. Put $r_{2}:=\min \{q, 3 / 2\}$. Then we may 
conclude that $\nabla V$ and $H$ are $L_{l o c}^{r_{2}}$-functions in $A$, so $V \in W_{l o c}^{2, r_{2}}(A)^{3}$ and $\Pi \in W_{l o c}^{1, r_{2}}(A)$ by Theorem 3.2. Thus we are done if $q \leq 3 / 2$, otherwise $V \in W_{l o c}^{2,3 / 2}(A)^{3} \subset W_{l o c}^{1,3}(A)^{3}$. Setting $r_{3}:=\min \{q, 3\}$, we thus have $\partial_{l} V, H \in L_{l o c}^{r_{3}}(A)^{3}(1 \leq l \leq 3)$. Another reference to Theorem 3.2 yields $V \in W_{l o c}^{r_{3}, 2}(A)^{3}$ and $\Pi \in W_{l o c}^{1, r_{3}}(A)$. This settles the case $q \leq 3$. Else $V \in W_{l o c}^{3,2}(A)^{3} \subset W_{l o c}^{1, q}(A)^{3}$, hence $H \in L_{l o c}^{q}(A)^{3}$, so Corollary 3.1 follows by once more referring to Theorem 3.2.

Concerning regularity near the boundary, we again start with a result about Stokes flows: $\langle$ theoremT4.40〉

Theorem 3.3 ([17, Lemma IV.6.1]) Let $A \subset \mathbb{R}^{3}$ be open and bounded, with $C^{2}$-boundary, $r \in(1, \infty), G \in L^{r}(A)^{3}, V \in W_{\text {loc }}^{1,1}(A)^{3}$ with $\nabla V \in L^{r}(A)^{9}$ (hence $V \in W^{1, r}(A)^{3}$ by Theorem 2.1), $V \mid \partial A \in W^{2-1 / r, r}(\partial A)^{3}$, div $V=0$, and with $V$ satifying (3.3) (weak solution of the Stokes system). Then $V \in W^{2, r}(A)^{3}$. Let $\Pi \in L_{\text {loc }}^{r}(A)$ be such that (3.4) is satisfied (associated pressure). Then $\Pi \in W^{1, r}(A)$ and $-\Delta V+\nabla \Pi=G$.

corollaryC4.20>

Corollary 3.2 Let $A \subset \mathbb{R}^{3}$ be open and bounded, with $C^{2}$-boundary, $s, q \in(1, \infty), \lambda \in$ $\mathbb{C}, G \in L^{q}(A)^{3}, V \in W_{\text {loc }}^{1,1}(A)^{3}$ with $\nabla V \in L^{s}(A)^{9}$ (hence $V \in W^{1, s}(A)^{3}$ by Theorem 2.1), $V \mid \partial A \in W^{2-1 / q, q}(\partial A)^{3}$, div $V=0$, and with $V$ satifying (3.1) ( $V$ weak solution of (3.5)). Then $V \in W^{2, q}(A)^{3}$. Let $\Pi \in L_{\text {loc }}^{s}(A)$ be a pressure associated with $V$ (Theorem 3.1). Then $\Pi \in W^{1, q}(A)$ and equation (3.5) holds.

Proof: We have $V \mid \partial A \in W^{2-1 / r, r}(\partial A)^{3}$ for $r \in[1, q]$. Thus we may proceed in the same iterative way as in the proof of Corollary 3.1, but with the references to Theorem 3.2 replaced by ones to Theorem 3.3 .

corollaryC4.30〉

Corollary 3.3 Let $s, q \in(1, \infty), \lambda \in \mathbb{C}, G \in L_{l o c}^{q}\left(\mathbb{R}^{3}\right)^{3}, V \in W_{\text {loc }}^{1,1}\left(\bar{\Omega}^{c}\right)^{3}$ with $\nabla V \mid \Omega_{R} \in$ $L^{s}\left(\Omega_{R}\right)^{9}$ (hence $V \mid \Omega_{R} \in W^{1, s}\left(\Omega_{R}\right)^{3}$ by Theorem 2.1) for any $R \in[S, \infty), V \mid \partial \Omega \in$ $W^{2-1 / q, q}(\partial \Omega)^{3}$, div $V=0$. Further suppose that $V$ satifies (3.1) with $A=\bar{\Omega}^{c}$. Then $V \mid \Omega_{R} \in W^{2, q}\left(\Omega_{R}\right)^{3}$ for $R \in[S, \infty)$. Let $\Pi \in L_{\text {loc }}^{s}\left(\bar{\Omega}^{c}\right)$ be a pressure associated with $V$ (Theorem 3.1). Then $\Pi \mid \Omega_{R} \in W^{1, q}\left(\Omega_{R}\right)$ for $R \in[S, \infty)$, and equation (3.5) holds.

Proof: Corollary 3.1 yields $V \in W_{l o c}^{2, q}\left(\bar{\Omega}^{c}\right)^{3}, \Pi \in W_{l o c}^{1, q}\left(\bar{\Omega}^{c}\right)$ as well as (3.5). Let $R \in$ $[S, \infty)$. Since $V \in W_{l o c}^{2, q}\left(\bar{\Omega}^{c}\right)^{3}$, we have $V \mid \partial B_{R} \in W^{2-1 / q, q}\left(\partial B_{R}\right)^{3}$. Thus, recalling the assumption on $V \mid \partial \Omega$, we obtain $V \mid \partial \Omega_{R} \in W^{2-1 / q, q}\left(\partial \Omega_{R}\right)^{3}$. Now Corollary 3.2 yields $V\left|\Omega_{R} \in W^{2, q}\left(\Omega_{R}\right)^{3}, \Pi\right| \Omega_{R} \in W^{1, q}\left(\Omega_{R}\right)$.

Next we present a criterion on $C^{\infty}$-regularity.

corollaryc4.40> Corollary 3.4 Let $A \subset \mathbb{R}^{3}$ be open, $q \in(1, \infty), \lambda \in \mathbb{C}, G \in C^{\infty}(A)^{3}, V \in W_{\text {loc }}^{1,1}(A)^{3}$ with $\nabla V \in L^{q}(A)^{9}$, div $V=0$, and with $V$ satisfying (3.1). Let $\Pi \in L_{\text {loc }}^{q}(A)$ be a pressure associated with $V$ (Theorem 3.1). Then $V \in C^{\infty}(A)^{3}, \Pi \in C^{\infty}(A)$ and equation (3.5) holds.

Proof: In the case $\lambda=0$, we may refer to [17, Theorem VII.1.1]. But both the case $\lambda=0$ and $\lambda \neq 0$ may be reduced to Theorem 3.2. In fact, by Corollary 3.1, we have $V \in W_{l o c}^{2, q}(A)^{3}, \Pi \in W_{l o c}^{1, q}(A)$, and equation (3.5) holds. Suppose that $n \in \mathbb{N}_{0}$ and $V \in W_{l o c}^{2+n, q}(A)^{3}, \Pi \in W_{l o c}^{1+n, q}(A)$. Then $-\mu \tau \partial_{1} V-\lambda V+G \in W_{l o c}^{1+n, q}(A)^{3}$, so Theorem 3.2 implies $V \in W_{l o c}^{3+n, q}(A)^{3}$ and $\Pi \in W_{l o c}^{2+n, q}(A)$. Therefore it follows by induction that 
$V \in W_{l o c}^{m, q}(A)^{3}$ and $\Pi \in W_{l o c}^{m-1, q}(A)$ for any $m \in \mathbb{N}, m \geq 2$. Corollary 3.4 follows by applying a Sobolev inequality to $V \mid B_{\epsilon}(x)$ and $\Pi \mid B_{\epsilon}(x)$, where $x$ is an arbitrary point in $A$ and $\epsilon>0$ is chosen in such a way that $\overline{B_{\epsilon}(x)} \subset A$.

We cite an existence result for weak solutions of the Oseen system $((3.5)$ with $\lambda=0)$.

$\langle$ theoremT4.50〉

Theorem 3.4 ([1, Proposition 4.2]) Let $A \subset \mathbb{R}^{3}$ be open and bounded, with $C^{2}$-boundary, $p \in(1, \infty), G \in W_{0}^{-1, p}(A)^{3}$. Then there is a unique function $V \in W_{0}^{1, p}(A)^{3}$ and a function $\Pi \in L^{q}(A)$, unique up to a constant, such that divu $=0$, and such that (3.2) holds with $\lambda=0$ (Oseen system) and $\int_{A} G \cdot \psi d x$ replaced by $G(\psi)$.

We are going to exploit this theorem in order to obtain weak solutions of the resolvent problem (3.5). These weak solutions have the special feature that the pressure belongs to a uniqueness class that will be convenient in what follows. A proof of this result is well known in principle: it makes use of the fact that on bounded domains, the resolvent term $\lambda V$ may be considered as a compact perturbation of the Laplace operator. However, since some details are perhaps less evident, and for the convenience of the reader, we indicate the main elements of this proof.

Corollary 3.5 Let $A \subset \mathbb{R}^{3}$ be open and bounded, with $C^{2}$-boundary. Let $\zeta \in C_{0}^{\infty}(A)$ with $\zeta \geq 0, \int_{A} \zeta d x>0$. Let $p \in(1, \infty)$. Then, for any $\lambda \in K_{\tau}, G \in W_{0}^{-1, p}(A)^{3}$, there is a unique pair of functions $(V, \Pi) \in W_{0}^{1, p}(A)^{3} \times L^{p}(A)$ such that $\int_{A} \zeta \Pi d x=0$, div $V=0$, and such that (3.2) holds with $\int_{A} G \cdot \psi d x$ replaced by $G(\psi)$. Moreover $\|V\|_{1, p}+\|\Pi\|_{p} \leq$ $\mathfrak{C}(p)\|G\|_{-1, p}$. If $p<3, s \in\left[p,(1 / p-1 / 3)^{-1}\right]$, we additionally have $\|V\|_{s} \leq \mathfrak{C}(p)\|G\|_{-1, p}$.

Proof: Put $W_{0, \sigma}^{1, p}:=\left\{W \in W_{0}^{1, p}(A)^{3}: \operatorname{div} W=0\right\}, L_{\zeta}^{p}:=\left\{\varphi \in L^{p}(A): \int_{A} \varphi \zeta d x=\right.$ $0\}, \mathfrak{W}_{p}:=W_{0, \sigma}^{1, p} \times L_{\zeta}^{p},\|\mid(V, \Pi)\|:=\|V\|_{1, p}+\|\Pi\|_{p}$ for $(V, \Pi) \in \mathfrak{W}_{p}$. Then $\mathfrak{W}_{p}$ is a vector space, the mapping $\left\||\|| \mid\right.$ is a norm on $\mathfrak{W}_{p}$, and $\mathfrak{W}_{p}$ equipped with this norm is a Banach space. Define $\mathfrak{F}: \mathfrak{W}_{p} \mapsto W_{0}^{-1, p}(A)^{3}$ by $\mathfrak{F}(V, \Pi)(\psi):=\int_{A}\left(\nabla V \cdot \nabla \psi+\mu \tau \partial_{1} V \cdot \psi-\Pi \operatorname{div} \psi\right) d x$ for $\psi \in W_{0}^{1, p^{\prime}}(A)^{3},(V, \Pi) \in \mathfrak{W}_{p}$. Obviously the operator $\mathfrak{F}$ is well defined, linear and bounded, and by Theorem 3.4, it is onto. In addition, due to this theorem and because the zero function is the only constant function in $L_{\zeta}^{p}$, this operator is one-to-one. Thus $\mathfrak{F}$ is bijective, so the open mapping theorem implies there is a constant $C_{0}>0$ with $\|V\|_{1, p}+\|\Pi\|_{p} \leq C_{0}\|\mathfrak{F}(V, \Pi)\|_{-1, p}$ for $(V, \Pi) \in \mathfrak{W}_{p}$. For $\varrho \in \mathbb{C}$, define $\mathfrak{K}_{\varrho}: \mathfrak{W}_{p} \mapsto W_{0}^{-1, p}(A)^{3}$ by $\mathfrak{K}_{\varrho}(V, \Pi)(\psi):=\int_{A} \varrho V \cdot \psi d x$ for $(V, \Pi) \in \mathfrak{W}_{p}, \psi \in W_{0}^{1, p^{\prime}}(A)^{3}$. This operator $\mathfrak{K}_{\varrho}$ is linear, bounded and compact. Let $\varrho \in \mathbb{C} \backslash\{0\}$ with $\Re \varrho \geq 0$. If $p \geq 2$, it may be shown by some partial integrations that $\Re\left(\mathfrak{F}+\mathfrak{K}_{\varrho}\right)(V, \Pi)(\bar{V})>0$ for $(V, \Pi) \in \mathfrak{W}_{p}$ with $V \neq 0$. Thus we get with Corollary 3.1 and 2.2 that the operator $\mathfrak{F}+\mathfrak{K}_{\varrho}$ is one-to-one if $p \geq 2$. Suppose that $p<2$ and the pair $(V, \Pi) \in \mathfrak{W}_{p}$ satisfies the equation $\left(\mathfrak{F}+\mathfrak{K}_{\varrho}\right)(V, \Pi)=0$. Then Corollary 3.2 yields $V \in W^{2, p^{\prime}}(A)^{3}, \Pi \in W^{1, p^{\prime}}(A)$. Therefore the term $\left(\mathfrak{F}+\mathfrak{K}_{\varrho}\right)(V, \Pi)(\bar{V})$ is well defined, and again by partial integration we may conclude this term is strictly positive if $V \neq 0$. As a consequence, the operator $\mathfrak{F}+\mathfrak{K}_{\varrho}$ is one-to-one in the case $p<2$, too. On the other hand, since $\mathfrak{F}$ is linear, bounded and bijective and $\mathfrak{K}_{\varrho}$ is linear and compact, the sum $\mathfrak{F}+\mathfrak{K}_{\varrho}$ is Fredholm with index zero. All these observations taken together imply that $\mathfrak{F}+\mathfrak{K}_{\varrho}$ is bijective, so by the open mapping theorem, there is a constant $C_{\varrho}>0$ with $\|V\|_{1, p}+\|\Pi\|_{p} \leq C_{\varrho}\left\|\left(\mathfrak{F}+\mathfrak{K}_{\varrho}\right)(V, \Pi)\right\|_{-1, p}$ for $(V, \Pi) \in \mathfrak{W}_{p}$. Recall we supposed $\varrho \in \mathbb{C} \backslash\{0\}$ with $\Re \varrho \geq 0$, and in the case $\varrho=0$, the preceding inequality was proved further above. 
As a consequence of these estimates, we get for $\varrho, \widetilde{\varrho} \in \mathbb{C}$ with $\Re \varrho \geq 0,(V, \Pi) \in \mathfrak{W}_{p}$ that $\left\|\left(\mathfrak{F}+\mathfrak{K}_{\widetilde{\varrho}}\right)(V, \Pi)\right\|_{-1, p} \geq\left\|\left(\mathfrak{F}+\mathfrak{K}_{\varrho}\right)(V, \Pi)\right\|_{-1, p}-|\varrho-\widetilde{\varrho}|\|V\|_{p} \geq\left(C_{\varrho}^{-1}-|\varrho-\widetilde{\varrho}|\right)\left(\|V\|_{1, p}+\|\Pi\|_{p}\right)$. Thus, for any $\varrho_{0} \in \mathbb{C}$ with $\Re \varrho_{0} \geq 0$, there is some $\epsilon\left(\varrho_{0}\right)$ such that for any $\varrho \in \mathbb{C}$ with $\left|\varrho-\varrho_{0}\right| \leq \epsilon\left(\varrho_{0}\right)$, we have $\|V\|_{1, p}+\|\Pi\|_{p} \leq 2 C_{\varrho_{0}}\left\|\left(\mathfrak{F}+\mathfrak{K}_{\varrho}\right)(V, \Pi)\right\|_{-1, p}$ for $(V, \Pi) \in \mathfrak{W}_{p}$. Now the first estimate at the end of Corollary 3.5 follows by an open covering argument, whereas the second may be deduced from the first by a Sobolev inequality.

We turn to strong solutions of (3.5) on bounded domains.

Theorem 3.5 Let $A \subset \mathbb{R}^{3}$ be open and bounded, with $C^{2}$-boundary, $q \in(1, \infty), \lambda \in$ $K_{\tau}, G \in L^{p}(A)^{3}$. Then there is a unique pair of functions $(V, \Pi) \in W^{2, q}(A)^{3} \times W^{1, q}(A)$

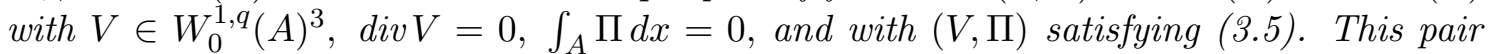
satifies the estimate $\|V\|_{2, q}+\|\Pi\|_{1, q} \leq \mathfrak{C}(q)\|G\|_{q}$.

Proof: A direct reference is [19, Proposition 2.6], where a much more detailed result is provided. But Theorem 3.5 may also be deduced by starting with an existence and a uniqueness result for strong solutions of the Oseen system on bounded domains (see [1, Proposition 4.3] for example), and then proceed with a perturbation argument as in the proof of Corollary 3.5.

Corollary 3.6 In the situation of the preceding theorem, we have $\|V\|_{p} \leq \mathfrak{C}(p, q, \epsilon)\|G\|_{q}$ for $p \in\left[1,(1 / q-2 / 3)^{-1}\right]$ if $q<3 / 2$, and for $p \in[1, \infty)$ if $q \geq 3 / 2 ;\|\nabla V\|_{p} \leq \mathfrak{C}(p, q, \epsilon)\|G\|_{q}$ for $p \in\left[1,(1 / q-1 / 3)^{-1}\right]$ if $q<3$, and for $p \in[1, \infty)$ if $q \geq 3$. Moreover, if $\lambda \neq 0, q \leq$ $2, p \in[q, 2]$, the estimate $\|V\|_{p} \leq \mathfrak{C}(p, q, \epsilon)|\lambda|^{-2+4(1 / q-1 / p)}\|G\|_{q}$ is valid.

Proof: The corollary follows from Theorem 3.5 and Sobolev estimates. Concerning the last estimate in the corollary, we note that $-2+4(1 / q-1 / p) \leq 0$ if $q \leq 2, p \in[q, 2]$, so that in the case $\lambda \neq 0$, we have $1 \leq C(p, q, \epsilon)|\lambda|^{-2+4(1 / q-1 / p)}$.

In the ensuing two theorems, we consider problem (1.8) (Oseen resolvent system in $\bar{\Omega}^{c}$, with Dirichlet boundary conditions), first for $\lambda=0$ (Oseen system), and then for $\lambda \neq 0$.

$\langle$ theoremT4.70〉

Theorem 3.6 ([17, Theorem VII.7.1]) Let $q \in(1,2), G \in L^{q}\left(\bar{\Omega}^{c}\right)^{3}$. Then there are functions $V \in W_{l o c}^{2, q}\left(\bar{\Omega}^{c}\right)^{3} \cap L^{(1 / q-1 / 2)^{-1}}\left(\bar{\Omega}^{c}\right)^{3}, \Pi \in W_{\text {loc }}^{1, q}\left(\bar{\Omega}^{c}\right)$ such that the relations $\nabla V \in$ $L^{(1 / q-1 / 4)^{-1}}\left(\bar{\Omega}^{c}\right)^{9}, \partial_{l} \partial_{j} V_{k}, \partial_{j} \Pi \in L^{q}\left(\bar{\Omega}^{c}\right)(1 \leq j, k, l \leq 3)$ hold, the pair $(V, \Pi)$ satisfies (1.8) with $\lambda=0$, and $\|V\|_{(1 / q-1 / 2)^{-1}}+\|\nabla V\|_{(1 / q-1 / 4)^{-1}}+\left\|D^{2} V\right\|_{q}+\|\nabla \Pi\|_{q} \leq \mathfrak{C}(q)\|G\|_{q}$.

Theorem 3.7 Let $p \in(1, \infty), \lambda \in \mathbb{C} \backslash\{0\}$ with $\Re \lambda \geq 0, G \in L^{p}\left(\bar{\Omega}^{c}\right)^{3}$. Then there is a unique function $V \in W^{2, p}\left(\bar{\Omega}^{c}\right)^{3} \cap W_{0}^{1, p}\left(\bar{\Omega}^{c}\right)^{3}$ and a function $\Pi \in W_{l o c}^{1, p}\left(\bar{\Omega}^{c}\right)$, unique up to a constant, such that $\nabla \Pi \in L^{p}\left(\bar{\Omega}^{c}\right)^{3}$, the pair $(V, \Pi)$ fulfills (1.8), and $\|V\|_{2, p}+\|\nabla \Pi\|_{p} \leq$ $\mathfrak{C}(p, \lambda)\|G\|_{p}$.

Proof: See [19, Theorem 4.4] and the proof of this theorem in [19, p. 29].

\section{Oseen resolvent estimates in the whole space $\mathbb{R}^{3}$.}

In this section, we extend some results from [11] concerning solutions to the Oseen resolvent (3.5) in the whole space $\mathbb{R}^{3}$. As in [11], our theory is based on the use of the 
fundamental solution $E^{(\lambda)}$ (see Section 2) of the scalar Oseen system. This approach has the inconvenient feature that the Helmholtz projection $P_{q}$ (Theorem 2.10) is involved when we represent solutions to (3.5) by means of convolutions with $E^{(\lambda)}$. However, although a fundamental solution to (3.5) is available $([19$, p. 19-20]), we were not able to estimate it in a satisfactory way. We begin by stating some basic facts about convolutions with $E^{(\lambda)}$, most of them taken from [11].

Theorem 4.1 Let $\lambda \in K_{\tau}$. Suppose that $H \in L^{p}\left(\mathbb{R}^{3}\right)$ for some $p \in(3, \infty)$ and for some $p \in(1,3 / 2)$. Let $\alpha \in \mathbb{N}_{0}^{3}$ with $|\alpha| \leq 1$. Then $\int_{\mathbb{R}^{3}}\left|\partial^{\alpha} E^{(\lambda)}(x-y)\right||H(y)| d y<\infty$ for any $x \in \mathbb{R}^{3}$.

Let $q \in[1,2)$ and $p \in\left((1 / q-1 / 2)^{-1}, \infty\right]$ if $q \geq 3 / 2$, or $p \in\left((1 / q-1 / 2)^{-1},(1 / q-2 / 3)^{-1}\right)$ if $q<3 / 2$, or $p=6$ if $q=6 / 5$. Then $\left\|\left|E^{(\lambda)}\right| *|G|\right\|_{p} \leq C(p, q, \tau)\|G\|_{q}$ for $G \in L^{q}\left(\mathbb{R}^{3}\right)$. In particular, the function $\left|E^{\lambda}(x-y)\right| *|G(y)|$ is integrable with respect to $y \in \mathbb{R}^{3}$, for a. $e$. $x \in \mathbb{R}^{3}$.

Let $q \in[1,3], p \in\left((1 / q-1 / 4)^{-1},(1 / q-1 / 3)^{-1}\right)$, or $p=2$ if $q=6 / 5$, and let $l \in$ $\{1,2,3\}$. Then $\left\|\left|\partial_{l} E^{(\lambda)}\right| *|G|\right\|_{p} \leq C(p, q, \tau)\|G\|_{q}$ for $G \in L^{q}\left(\mathbb{R}^{3}\right)$. In particular, the function $\left|\partial_{l} E^{\lambda}(x-y)\right| *|G(y)|$ is integrable with respect to $y \in \mathbb{R}^{3}$, for a. e. $x \in \mathbb{R}^{3}$.

If $\lambda \neq 0, q \in[1,2], p \in[q, 2]$ and $q>1$ or $p<2$, then

$$
\left\|\left|E^{(\lambda)}\right| *|G|\right\|_{p} \leq C(p, q, \tau)|\lambda|^{-2+4(1 / q-1 / p)}\|G\|_{q} \quad \text { for } G \in L^{q}\left(\mathbb{R}^{3}\right) .
$$

Also for such $p$ and $q$, the function $\left|E^{\lambda}(x-y)\right| *|G(y)|$ is integrable with respect to $y \in \mathbb{R}^{3}$, for a. e. $x \in \mathbb{R}^{3}$.

Let $\phi \in C_{0}^{\infty}\left(\mathbb{R}^{3}\right)$, and put $V:=E^{(\lambda)} * \phi$. Then $V \in C^{\infty}\left(\mathbb{R}^{3}\right), \partial^{\beta} V=E^{(\lambda)} * \partial^{\beta} \phi$ for $\beta \in \mathbb{N}_{0}^{3}, \partial_{l} V=\left(\partial_{l} E^{(\lambda)}\right) * \phi$ for $1 \leq l \leq 3,-\Delta V+\tau \partial_{1} V+\lambda V=\phi$, and $\left\|D^{2} V \mid B_{R}\right\|_{q} \leq$ $C(R, q, \tau)\|\phi\|_{q}$ for $q \in(1, \infty), R \in(0, \infty)$.

Proof: We have $\left|\partial^{\alpha} E^{(\lambda)}(z)\right| \leq C(\tau)\left(\chi_{(0,1)}(|z|)|z|^{-1-|\alpha|}+\chi_{[1, \infty)}(|z|)|z|^{-1-|\alpha| / 2}\right.$ for $z \in$ $\mathbb{R}^{3} \backslash\{0\}, \alpha \in \mathbb{N}_{0}^{3}$ with $|\alpha| \leq 1$ by [11, Theorem 9$]$. Let $x \in \mathbb{R}^{3}$, and take $\alpha$ as before. Then, by Hölder's inequality and the preceding estimate, the function $\left|\partial^{\alpha} E^{(\lambda)}\right||H(y)|$ is integrable with respect to $y \in B_{1}(x)$ because $H \in L^{p}\left(\mathbb{R}^{3}\right)$ for some $p \in(3, \infty)$, and with respect to $y \in B_{1}(x)^{c}$ since $H \in L^{p}\left(\mathbb{R}^{3}\right)$ for some $p \in(1,3 / 2)$. The inequalities stated in the theorem, except the last one, hold according to [11, Theorem 10]. As for the last part of the theorem, pertaining to the function $V$, we refer to [11, Theorem 11].

$\left\langle\right.$ theoremT5.20〉 Theorem 4.2 Let $\lambda \in K_{\tau}$ and $q \in(1, \infty)$. Then $\left\|D^{2}\left(E^{(\lambda)} * \phi\right)\right\|_{q} \leq C(q, \tau)\|\phi\|_{q}$ for $\phi \in C_{0}^{\infty}\left(\mathbb{R}^{3}\right)$ if $\lambda=0$ or if $q=2$. This means in particular that in the case $q=2$, the constant $C(q, \tau)$ does not depend on $\lambda$, even if $\lambda \neq 0$. In that latter case, we further have $\left\|E^{(\lambda)} * \phi\right\|_{1, q} \leq C(q, \lambda, \tau)\|\phi\|_{q}$ for $\phi \in C_{0}^{\infty}\left(\mathbb{R}^{3}\right)$.

Proof: For $f \in L^{1}\left(\mathbb{R}^{3}\right)$, define $\hat{f}(\xi):=(2 \pi)^{-3 / 2} \int_{\mathbb{R}^{3}} e^{-i \xi \cdot \eta} f(\eta) d \eta$ for $\xi \in \mathbb{R}^{3}$ (Fourier transform of $f)$. Let $\phi \in C_{0}^{\infty}\left(\mathbb{R}^{3}\right), l, m \in\{1,2,3\}$. Then $\partial_{l} \partial_{m}\left(E^{(\lambda)} * \phi\right)=E^{(\lambda)} *\left(\partial_{l} \partial_{m} \phi\right)$ by Theorem 4.1, so we get from [13, proof of Corollary 4.1] in the case $\lambda=0$, and from [12, Theorem 2.1] if $\lambda \neq 0$ that $\partial_{l} \partial_{m}\left(E^{(\lambda)} * \phi\right)(x)=\int_{\mathbb{R}^{3}} e^{i x \cdot \eta}(2 \pi)^{-3 / 2}\left(\lambda+|\eta|^{2}+\right.$ $\left.i \tau \eta_{1}\right)^{-1} \eta_{l} \eta_{m} \hat{\phi}(\eta) d \eta$ for $x \in \mathbb{R}^{3}$. In this situation it is shown in [17, p. 447-450] (in particular see [17, (VII.4.14)]), on the basis of Lizorkin's multiplier theorem, that the first estimate in Theorem 4.2 is valid in the case $\lambda=0$. Now suppose that $\lambda \neq 0$. We 
have $\left|\eta_{l} \eta_{m}\left(\lambda+|\eta|^{2}+i \tau \eta_{1}\right)^{-1}\right| \leq C$ for $\eta \in \mathbb{R}^{3} \backslash\{0\}$ because $\Re \lambda \geq 0$. This estimate combined with the equations $\partial_{l} \partial_{m}\left(E^{(\lambda)} * \phi\right)=E^{(\lambda)} *\left(\partial_{l} \partial_{m} \phi\right)$ and $\widehat{E^{(\lambda)}}(\eta)=(2 \pi)^{-3 / 2}(\lambda+$ $\left.|\eta|^{2}+i \tau \eta_{1}\right)^{-1}\left(\eta \in \mathbb{R}^{3} \backslash\{0\}\right)([12$, Theorem 2.1]) and with Plancherel's theorem yield the first estimate in Theorem 4.2 for $q=2$. According to [11, (3.2)], we have $\left|\partial^{\alpha} E^{(\lambda)}(z)\right| \leq$ $C(\tau, \lambda)\left(\chi_{(0,1)}(|z|)|z|^{-1-|\alpha|}+\chi_{[1, \infty)}(|z|) e^{-\sigma|\lambda|^{2}|z|}\right)$ for $z \in \mathbb{R}^{3} \backslash\{0\}, \alpha \in \mathbb{N}_{0}^{3}$ with $|\alpha| \leq 1$, where $\sigma>0$ is a constant independent of $z$ and $\lambda$. It follows that $\partial^{\alpha} E^{(\lambda)} \in L^{1}\left(\mathbb{R}^{3}\right)$ for $\alpha$ as before. Thus, by Young's inequality ([22, Part I, Theorem I.2]), we get $\left\|\left(\partial^{\alpha} E^{(\lambda)}\right) * \phi\right\|_{q} \leq$ $C(\tau, \lambda)\|\phi\|_{q}$, for $\alpha$ as before and for $\phi \in C_{0}^{\infty}\left(\mathbb{R}^{3}\right)$, so $\left\|E^{(\lambda)} * \phi\right\|_{1, q} \leq C(\tau, \lambda)\|\phi\|_{q}$.

Corollary 4.1 Let $\lambda \in K_{\tau}$ and $G \in C_{0}^{\infty}\left(\mathbb{R}^{3}\right)^{3}$. Then $P_{2}(G) \in L^{p}\left(\mathbb{R}^{3}\right)^{3}$ for $p \in(1, \infty)$, so we may define $V:=E^{(\lambda)} * P_{2}(G)$. Put $\Pi:=Q_{2}(G)$ (Theorem 2.10). Then $V \in$ $C^{\infty}\left(\mathbb{R}^{3}\right)^{3}, \Pi \in C^{\infty}\left(\mathbb{R}^{3}\right)$, and (3.5) holds.

In addition $\|V\|_{p} \leq C(p, q, \tau)\|G\|_{q},\|\nabla V\|_{p} \leq C(p, q, \tau)\|G\|_{q}$, with the range of $p$ and $q$ being the same as in the corresponding estimates in Theorem 4.1, except for the additional restriction $q>1$.

Moreover, $\left\|D^{2} V \mid B_{R}\right\|_{q} \leq C(q, R, \tau)\|G\|_{q}$ for $q \in(1, \infty), \quad R \in(0, \infty)$, and $\left\|D^{2} V\right\|_{2} \leq$ $C(\tau)\|G\|_{2}$. If $\lambda=0$, the inequality $\left\|D^{2} V\right\|_{q} \leq C(q, \tau)\|G\|_{q}$ holds for $q \in(1, \infty)$.

If $\lambda \neq 0$, we further have $\|V\|_{1, q} \leq C(q, \lambda, \tau)\|G\|_{q}$ for $q \in(1, \infty)$. Moreover the estimate $\|V\|_{p} \leq C(p, q, \tau)|\lambda|^{-2+4(1 / q-1 / p)}\|G\|_{q}$ is valid for $p, q$ as in the corresponding inequality in Theorem 4.1, but with the additional condition $q>1$. Finally $\|\nabla \Pi\|_{q} \leq C(q)\|G\|_{q}$ for $q \in(1, \infty)$.

Proof: From Theorem 2.10 and $(2.3)$, we get $P_{2}(G), Q_{2}(G) \in C^{\infty}\left(\mathbb{R}^{3}\right)$. It further follows from Theorem 2.10 and Corollary 2.4 that $P_{2}(G) \in H_{q}\left(\mathbb{R}^{3}\right)$ and $\left\|\nabla Q_{2}(G)\right\|_{q} \leq C(q)\|G\|_{q}$ for $q \in(1, \infty)$. In particular the last inequality in Corollary 4.1 is valid. Let $p \in(1, \infty)$. By Lemma 2.1, we may choose a sequence $\left(\phi_{n}\right)$ in $C_{0}^{\infty}\left(\mathbb{R}^{3}\right)^{3}$ with $\left\|P_{2}(G)-\phi_{n}\right\|_{6 / 5} \rightarrow 0$ and $\left\|P_{2}(G)-\phi_{n}\right\|_{p} \rightarrow 0$. By Theorem 4.1 with $q=6 / 5$, we have $\left\|V-E^{(\lambda)} * \phi_{n}\right\|_{6} \rightarrow 0$. The same reference yields $\left\|\left(\partial_{l} E^{(\lambda)}\right) * P_{2}(G)-\partial_{l}\left(E^{(\lambda)} * \phi_{n}\right)\right\|_{2} \rightarrow 0$ for $1 \leq l \leq 3$. Again by Theorem 4.1, we see that the sequence $\left(D^{2}\left(E^{(\lambda)} * \phi_{n}\right) \mid B_{R}\right)_{n>0}$ converges in $L^{p}\left(B_{R}\right)^{27}$, for any $R \in(0, \infty)$. These relations imply that $V \in W_{l o c}^{2,1}\left(\mathbb{R}^{3}\right)^{3}, \partial_{l} V=\left(\partial_{l} E^{(\lambda)}\right) * P_{2}(G)$ for $1 \leq l \leq 3$, and $\left\|D^{2} V-D^{2}\left(E^{(\lambda)} * \phi_{n}\right) \mid B_{R}\right\|_{p} \rightarrow 0(n \rightarrow \infty)$ for any $R \in(0, \infty)$. Now we may conclude from Theorem 4.1 that $-\Delta V+\tau \partial_{1} V+\lambda V=P_{2}(G)$. The first equation in (3.5) now follows by Theorem 2.10 and the definition of $\Pi$. Since $P_{2}(G) \in H_{6 / 5}\left(\mathbb{R}^{3}\right)$ (Corollary 2.4), we may choose a sequence $\left(\psi_{n}\right)$ in $C_{0}^{\infty}\left(\mathbb{R}^{3}\right)^{3}$ with $\operatorname{div} \psi_{n}=0$ for $n \in \mathbb{N}$ and $\left\|P_{2}(G)-\psi_{n}\right\|_{6 / 5} \rightarrow 0$. But $\partial_{l} V=\left(\partial_{l} E^{(\lambda)}\right) * P_{2}(G)$ (see above), so we may deduce from Theorem 4.1 with $q=6 / 5$ that $\left\|\partial_{l} V-\left(\partial_{l} E^{(\lambda)}\right) * \psi_{n}\right\|_{2} \rightarrow 0$ for $1 \leq l \leq 3$. We again refer to Theorem 4.1 to obtain $\operatorname{div}\left(E^{(\lambda)} * \psi_{n}\right)=E^{(\lambda)} * \operatorname{div} \psi_{n}=0$ for $n \in \mathbb{N}$. Thus we get $\operatorname{div} V=0$, so (3.5) is proved. Corollary 3.4 now yields $V \in C^{\infty}\left(\mathbb{R}^{3}\right)$. The estimates of $\|V\|_{p}$ and $\|\nabla V\|_{p}$ claimed in the corollary follow from Theorem 4.1, 2.10, Corollary 2.4 and the equation $\partial_{l} V=\left(\partial_{l} E^{(\lambda)}\right) * P_{2}(G)$ proved above. As concerns the estimates of $\left\|D^{2} V \mid B_{R}\right\|_{q},\left\|D^{2} V\right\|_{2}$ and $\left\|D^{2} V\right\|_{q}$, we refer to Theorem 4.1, 4.2, 2.10 and Corollary 2.4 , and to the approximation of $D^{2} V \mid B_{R}$ shown above. Finally, if $\lambda \neq 0$, the estimate of $\|V\|_{1, q}$ follows from Theorem 4.2, 2.10 and Corollary 2.4.

Corollary 4.2 Let $\lambda \in K_{\tau}$ and $q \in(1, \infty)$. Suppose that $\lambda=0$ or $q=2$. Let $p \in$ 
$(1,2), \phi \in W_{l o c}^{1,1}\left(\mathbb{R}^{3}\right) \cap L^{q}\left(\mathbb{R}^{3}\right)$ with $\nabla \phi \in L^{p}\left(\mathbb{R}^{3}\right)^{3}, \quad l \in\{1,2,3\}$. Then $E^{(\lambda)} * \partial_{l} \phi \in$ $W_{l o c}^{1,1}\left(\mathbb{R}^{3}\right)$ and $\left\|\partial_{m}\left(E^{(\lambda)} * \partial_{l} \phi\right)\right\|_{q} \leq C(q, \tau)\|\phi\|_{q}(1 \leq m \leq 3)$. If $q<3$, we additionally have $E^{(\lambda)} * \partial_{l} \phi=\left(\partial_{l} E^{(\lambda)}\right) * \phi$.

Proof: By Lemma 2.1, we may choose a sequence $\left(\phi_{n}\right)$ in $C_{0}^{\infty}\left(\mathbb{R}^{3}\right)$ such that $\left\|\phi_{n}-\phi\right\|_{q} \rightarrow 0$ and $\left\|\nabla\left(\phi_{n}-\phi\right)\right\|_{p} \rightarrow 0$. Fix some $r \in\left((1 / p-1 / 2)^{-1}, \infty\right)$ with $r<(1 / p-2 / 3)^{-1}$ in the case $p<3 / 2$. Then $\left\|E^{(\lambda)} * \partial_{l} \phi-E^{(\lambda)} * \partial_{l} \phi_{n}\right\|_{r} \rightarrow 0$ by Theorem 4.1 , and $\| \partial_{m} \partial_{l}\left(E^{(\lambda)} * \phi_{n}-E^{(\lambda)} *\right.$ $\left.\phi_{k}\right)\left\|_{q} \leq C(q)\right\| \phi_{n}-\phi_{k} \|_{q}$ for $n, k \in \mathbb{N}, 1 \leq m \leq 3$ by Theorem 4.2. The latter inequality implies that the sequence $\left(\partial_{m} \partial_{l}\left(E^{(\lambda)} * \phi_{n}\right)\right)_{n>0}$ converges in $L^{q}\left(\mathbb{R}^{3}\right)$, for $1 \leq m \leq 3$. Moreover $\partial_{m} \partial_{l}\left(E^{(\lambda)} * \phi_{n}\right)=\partial_{m}\left(E^{(\lambda)} * \partial_{l} \phi_{n}\right)$ for $n \in \mathbb{N}, 1 \leq m \leq 3$, again by Theorem 4.1. Thus we may conclude that $E^{(\lambda)} * \partial_{l} \phi \in W_{l o c}^{1,1}\left(\mathbb{R}^{3}\right), \partial_{m}\left(E^{(\lambda)} * \partial_{l} \phi\right) \in L^{q}\left(\mathbb{R}^{3}\right)$ and $\left\|\partial_{m} \partial_{l}\left(E^{(\lambda)} * \phi_{n}\right)-\partial_{m}\left(E^{(\lambda)} * \partial_{l} \phi\right)\right\|_{q} \rightarrow 0$. Since $\left\|\partial_{m} \partial_{l}\left(E^{(\lambda)} * \phi_{n}\right)\right\|_{q} \leq C(q, \tau)\left\|\phi_{n}\right\|_{q}(n \in$ $\mathbb{N}, 1 \leq m \leq 3)$ by Theorem 4.2, we obtain the estimate stated in Corollary 4.2. Now suppose that $q<3$. Choose $s \in\left((1 / q-1 / 4)^{-1},(1 / q-1 / 3)^{-1}\right)$. By Theorem 4.1, we get $\left\|\left(\partial_{l} E^{(\lambda)}\right) * \phi-\left(\partial_{l} E^{(\lambda)}\right) * \phi_{n}\right\|_{s} \rightarrow 0$. On the other hand, $\left(\partial_{l} E^{(\lambda)}\right) * \phi_{n}=E^{(\lambda)} * \partial_{l} \phi_{n}$ for $n \in \mathbb{N}$ by Theorem 4.1. Since $E^{(\lambda)} * \partial_{l} \phi_{n} \rightarrow E^{(\lambda)} * \partial_{l} \phi$ in $L^{r}\left(\mathbb{R}^{3}\right)^{3}$, as noted above, we thus obtain the equation stated at the end of Corollary 4.2 .

For the proof of the ensuing theorem, we adapt an approach from [17, p. 454-456].

$\langle$ theoremT5.30〉

Theorem 4.3 Let $\lambda \in K_{\tau}$ and $q \in(1, \infty)$, with $\lambda=0$ or $q=2$. Let $G \in C_{0}^{\infty}\left(\mathbb{R}^{3}\right)^{3} \cap$ $\widetilde{\mathcal{D}}_{0}^{-1, q}\left(\mathbb{R}^{3}\right)^{3}$. (According to Corollary 2.1, in the case $q>3 / 2$, it suffices to require $G \in$ $\left.C_{0}^{\infty}\left(\mathbb{R}^{3}\right)^{3}\right)$. Then $\left\|\partial_{k}\left(E^{(\lambda)} * P_{2}(G)\right)\right\|_{q} \leq C(q, \tau)\|G\|_{-1, q}(1 \leq k \leq 3)$. If $q<3$ and $s \in\left((1 / q-1 / 4)^{-1},(1 / q-1 / 3)^{-1}\right)$, we further have $\left\|E^{(\lambda)} * P_{2}(G)\right\|_{s} \leq C(q, s, \tau)\|G\|_{-1, q}$.

Proof: Corollary 2.4 yields $P_{2}(G) \in L^{r}\left(\mathbb{R}^{3}\right)^{3}$ for any $r \in(1, \infty)$. By referring to $(2.3)$ and to Corollary 2.3 with $q$ in the place of $p$, we get for $\psi \in C_{0}^{\infty}\left(\mathbb{R}^{3}\right)^{3}, 1 \leq l \leq 3$ that

$$
\begin{aligned}
& \left|\int_{\mathbb{R}^{3}}\left(\left(\partial_{l} N\right) * \operatorname{div} G\right) \psi d x\right|=\left|\int_{\mathbb{R}^{3}}\left(\left(\partial_{l} N\right) * G\right) \cdot \nabla \psi d x\right| \leq\left\|\left(\partial_{l} N\right) * G\right\|_{q}\|\nabla \psi\|_{q^{\prime}} \\
& \leq C(q)\|G\|_{-1, q}\|\nabla \psi\|_{q^{\prime}} .
\end{aligned}
$$

Hence $\left\|\left(\partial_{l} N\right) * \operatorname{div} G\right\|_{-1, q} \leq C(q)\|G\|_{-1, q}$ for $1 \leq l \leq 3$. Thus we may conclude from Theorem 2.10 that $\left\|P_{2}(G)\right\|_{-1, q} \leq C(q)\|G\|_{-1, q}$. Again referring to Corollary 2.3, we then arrive at the estimate

$$
\left\|\left(\partial_{l} N\right) * P_{2}(G)\right\|_{q} \leq C(q, \tau)\|G\|_{-1, q}(1 \leq l \leq 3) .
$$

By the first sentence of this proof and by Theorem 2.9, we know that $N * P_{2}(G) \in$ $W_{l o c}^{2,1}\left(\mathbb{R}^{3}\right)^{3},\left(\partial_{l} N\right) * P_{2}(G)=\partial_{l}\left(N * P_{2}(G)\right), D^{2}\left(N * P_{2}(G)\right) \in L^{p}\left(\mathbb{R}^{3}\right)^{27}$ for $p>1$, and

$$
\operatorname{div}\left(\left(\partial_{l} N\right) * P_{2}(G)_{j}\right)_{1 \leq l \leq 3}=P_{2}(G)_{j} \text { for } 1 \leq j \leq 3 .
$$

We may conclude with (4.1) that

$$
\left(\partial_{l} N\right) * P_{2}(G) \in W_{l o c}^{1,1}\left(\mathbb{R}^{3}\right)^{3} \cap L^{q}\left(\mathbb{R}^{3}\right)^{3}, \nabla\left(\left(\partial_{l} N\right) * P_{2}(G)\right) \in L^{p}\left(\mathbb{R}^{3}\right)^{9}
$$

for any $p \in(1, \infty)$. Thus we may apply Corollary 4.2 with $\phi$ replaced by $\left(\partial_{l} N\right) * P_{2}(G)$. 
By (4.2), this corollary and (4.1), we get

$$
\begin{aligned}
& \left\|\partial_{k}\left(E^{(\lambda)} * P_{2}(G)\right)\right\|_{q} \leq \sum_{l=1}^{3}\left\|\partial_{k}\left[E^{(\lambda)} * \partial_{l}\left(\left(\partial_{l} N\right) * P_{2}(G)\right)\right]\right\|_{q} \\
& \leq C(q) \sum_{l=1}^{3}\left\|\left(\partial_{l} N\right) * P_{2}(G)\right\|_{q} \leq C(q)\|G\|_{-1, q} \quad(1 \leq k \leq 3),
\end{aligned}
$$

so the first estimate in Theorem 4.3 is proved. Now suppose that $q<3$ and $s \in((1 / q-$ $\left.1 / 4)^{-1},(1 / q-1 / 3)^{-1}\right)$. Then Corollary 4.2 and (4.3) yield $E^{(\lambda)} * \partial_{l}\left(\left(\partial_{l} N\right) * P_{2}(G)\right)=$ $\left(\partial_{l} E^{(\lambda)}\right) *\left(\left(\partial_{l} N\right) * P_{2}(G)\right)$, so the last inequality in Theorem 4.3 follows from Theorem 4.1 and (4.1), by an estimate as in (4.4).

$\langle$ lemmaL5.10〉

Lemma 4.1 Let $q \in(1, \infty), G \in C_{0}^{\infty}\left(\mathbb{R}^{3}\right)^{3} \cap \widetilde{\mathcal{D}}_{0}^{-1, q}\left(\mathbb{R}^{3}\right)^{3}$. Then $\left\|Q_{2}(G)\right\|_{q} \leq C(q)\|G\|_{-1, q}$.

Proof: Combine Theorem 2.10, the equation $N * \operatorname{div} G=\sum_{l=1}^{3}\left(\partial_{l} N\right) * G_{l}$ (see (2.3)) and Corollary 2.3.

The results of this section imply the following existence results for solutions to the Oseen system and to the Oseen resolvent system (3.5).

Corollary 4.3 Let $\lambda \in K_{\tau}$ and $G \in C_{0}^{\infty}\left(\mathbb{R}^{3}\right)^{3}$. Put $V:=E^{(\lambda)} * P_{2}(G)$, $\Pi:=Q_{2}(G)$. Then $V \in C^{\infty}\left(\mathbb{R}^{3}\right)^{3}, \Pi \in C^{\infty}\left(\mathbb{R}^{3}\right)$, and the pair $(V, \Pi)$ solves (3.5). Moreover, if $\lambda \neq 0$, we have $V \in W^{1, p}\left(\mathbb{R}^{3}\right)$ for any $p \in(1, \infty)$, and $D^{2} V \in L^{2}\left(\mathbb{R}^{3}\right)^{27}$. If $\lambda=0$, we have $V \in L^{p}\left(\mathbb{R}^{3}\right)^{3}$ for $p \in(2, \infty), \nabla V \in L^{p}\left(\mathbb{R}^{3}\right)^{9}$ for $p \in(4 / 3, \infty)$, and $D^{2} V \in L^{p}\left(\mathbb{R}^{3}\right)^{27}$ for $p \in(1, \infty)$. Moreover $\Pi \in L^{p}\left(\mathbb{R}^{3}\right)$ for $p \in(3 / 2, \infty)$ and $\nabla \Pi \in L^{p}\left(\mathbb{R}^{3}\right)^{3}$ for $p \in(1, \infty)$.

If $\lambda=0$ and $G \in C_{0}^{\infty}\left(\mathbb{R}^{3}\right)^{3} \cap \widetilde{\mathcal{D}}_{0}^{-1, p}\left(\mathbb{R}^{3}\right)^{3}$ for any $p \in(1, \infty)$, we get $V \in L^{p}\left(\mathbb{R}^{3}\right)^{3}$ for $p \in$ $(4 / 3, \infty)$ and $\nabla V \in L^{p}\left(\mathbb{R}^{3}\right)^{9}$ for $p \in(1, \infty)$. Moreover, if again $G \in C_{0}^{\infty}\left(\mathbb{R}^{3}\right)^{3} \cap \widetilde{\mathcal{D}}_{0}^{-1, p}\left(\mathbb{R}^{3}\right)^{3}$ for any $p \in(1, \infty)$, but with non restriction on $\lambda$, we get $\Pi \in L^{p}\left(\mathbb{R}^{3}\right)$ for such $p$.

Proof: According to Corollary 4.1, we have $V_{j}, \Pi \in C^{\infty}\left(\mathbb{R}^{3}\right)$ for $1 \leq j \leq 3$, and the pair $(V, \Pi)$ solves $(3.5)$. If $\lambda \neq 0$, Corollary 4.1 yields that $V \in W^{1, p}\left(\mathbb{R}^{3}\right)^{3}$ for $p \in(1, \infty)$ and $D^{2} V \in L^{2}\left(\mathbb{R}^{3}\right)^{27}$. In the case $\lambda=0$, the integrability relations stated for $V, \nabla V$ and $D^{2} V$ follow from Corollary 4.1, and if $G \in C_{0}^{\infty}\left(\mathbb{R}^{3}\right)^{3} \cap \widetilde{\mathcal{D}}_{0}^{-1, p}\left(\mathbb{R}^{3}\right)^{3}$ for $p \in(1, \infty)$, from Theorem 4.3. Due to Theorem 2.10, (2.3) and the second last statement of Theorem 2.9, we know that $\Pi \in L^{p}\left(\mathbb{R}^{3}\right)$ for $p \in(3 / 2, \infty)$. In the case $G \in C_{0}^{\infty}\left(\mathbb{R}^{3}\right)^{3} \cap \widetilde{\mathcal{D}}_{0}^{-1, p}\left(\mathbb{R}^{3}\right)^{3}$ for $p \in(1, \infty)$, Lemma 4.1 yields $\Pi \in L^{p}\left(\mathbb{R}^{3}\right)$ for this range of $p$. $L^{p}$-integrability of $\nabla \Pi$ for any $p \in(1, \infty)$ holds according to Corollary 4.1 .

\section{Uniqueness theorems for solutions to (1.8).}

We first consider the whole space case, then exploit the results we obtain for this case to determine a uniqueness class for weak solutions of (1.8) (exterior domain case). Our results are more general than what is available in literature because we do not suppose that the gradient of the velocity is an $L^{p}$-function for a single $p \in(1, \infty)$. Instead it may 


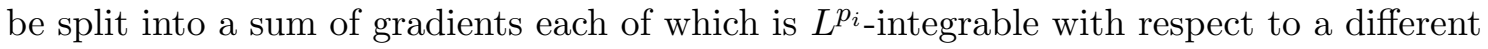
exponent $p_{i} \in(1, \infty)$. Unfortunately this seemingly small generalization complicates the argument considerably. For technical reasons, we first consider a splitting into a sum of three terms (whole space case), and then into a sum of two terms (exterior domain case).

Theorem 5.1 Let $\lambda \in K_{\tau}$. For $i \in\{1,2,3\}$, let $q_{i}, r_{i} \in(1, \infty), R_{i} \in(0, \infty)$ and $V^{(i)} \in$ $W_{\text {loc }}^{1,1}\left(\mathbb{R}^{3}\right)^{3}$ with $V^{(i)} \mid B_{R_{i}}^{c} \in L^{r_{i}}\left(B_{R_{i}}^{c}\right)^{3}, \nabla V^{(i)} \in L^{q_{i}}\left(\mathbb{R}^{3}\right)^{9}$. Suppose that div $\left(\sum_{i=1}^{3} V^{(i)}\right)=0$ and (3.1) is satisfied with $A=\mathbb{R}^{3}, G=0, V=\sum_{i=1}^{3} V^{(i)}$. Then $\sum_{i=1}^{3} V^{(i)}=0$.

Proof: Abbreviate $V:=\sum_{i=1}^{3} V^{(i)}, q:=\min \left\{q_{1}, q_{2}, q_{3}\right\}$. Then $V \in W_{l o c}^{1,1}\left(\mathbb{R}^{3}\right)^{3}, \nabla V \in$ $L_{l o c}^{q}\left(\mathbb{R}^{3}\right)^{9}$, div $V=0$, and (3.1) is satisfied with $A=\mathbb{R}^{3}, G=0$. Corollary 3.4 yields that $V \in C^{\infty}\left(\mathbb{R}^{3}\right)^{3}$, and that there is $\Pi \in C^{\infty}\left(\mathbb{R}^{3}\right)$ such that $(3.5)$ is valid with $G=0$.

Fix some function $\varphi \in C_{0}^{\infty}\left(B_{7 / 4}\right)^{3}$ with $\varphi \mid B_{5 / 4}=1,0 \leq \varphi \leq 1$, and put $\varphi_{n}(x):=$ $\varphi((1 / n) x)$ for $n \in \mathbb{N}, x \in \mathbb{R}^{3}$. Note that $\varphi_{n} \in C_{0}^{\infty}\left(B_{7 n / 4}\right), 0 \leq \varphi_{n} \leq 1, \varphi \mid B_{5 n / 4}=1$,

$$
|\nabla \varphi|_{\infty} \leq \mathfrak{C} n^{-1}, \quad\left|\partial_{l} \partial_{m} \varphi\right|_{\infty} \leq \mathfrak{C} n^{-2} \text { for } n \in \mathbb{N}, 1 \leq l, m \leq 3 .
$$

Let $l \in\{1,2,3\}, \phi \in C_{0}^{\infty}\left(\mathbb{R}^{3}\right)^{3}$. Obviously $\partial_{l} \phi \in C_{0}^{\infty}\left(\mathbb{R}^{3}\right)^{3} \cap \widetilde{\mathcal{D}}_{0}^{-1, p}\left(\mathbb{R}^{3}\right)^{3}$ for any $p \in(1, \infty)$. Therefore, according to Corollary 4.3, we may choose functions $W \in C^{\infty}\left(\mathbb{R}^{3}\right)^{3}, \Gamma \in$ $C^{\infty}\left(\mathbb{R}^{3}\right)$ such that the pair $(W, \Gamma)$ solves (3.5) with $W, \Gamma, \partial_{l} \phi,-\mu \tau$ in the place of $V, \Pi, G$, $\mu \tau$, respectively, and such that $W \in L^{p}\left(\mathbb{R}^{3}\right)^{3}$ for $p \in(4 / 3, \infty), \partial_{j} W_{k}, \Gamma \in L^{p}\left(\mathbb{R}^{3}\right)$ for $p \in(1, \infty), 1 \leq j, k \leq 3$. In the case $\lambda \neq 0$, we may require $W \in L^{p}\left(\mathbb{R}^{3}\right)^{3}$ even for any $p \in(1, \infty)$. On taking account of the fact that $\phi$ has compact support, we get

$$
\begin{aligned}
& \int_{\mathbb{R}^{3}} \partial_{l} V \cdot \phi d x=-\int_{\mathbb{R}^{3}} V \cdot \partial_{l} \phi d x=-\lim _{n \rightarrow \infty} \int_{\mathbb{R}^{3}} V \cdot \partial_{l} \phi \varphi_{n} d x \\
& =-\lim _{n \rightarrow \infty} \int_{\mathbb{R}^{3}} V \cdot\left(-\Delta W-\mu \tau \partial_{1} W+\lambda W+\nabla \Gamma\right) \varphi_{n} d x .
\end{aligned}
$$

Let $n \in \mathbb{N}$, and abbreviate $A_{n}:=B_{2 n} \backslash \overline{B_{n}}$. Since $\operatorname{div} W=0$ and $\operatorname{supp}\left(\nabla \varphi_{n}\right) \subset A_{n}$, we have $\nabla \varphi_{n} \cdot W \in C_{0}^{\infty}\left(A_{n}\right)$ and $\int_{A_{n}} \nabla \varphi_{n} \cdot W d x=0$. Thus we may refer to Theorem 2.7 and Lemma 2.3 to define $\mathfrak{D}_{n}:=n \mathfrak{D}(2,1,1,2)\left[\left(-\nabla \varphi_{n} \cdot W\right) \circ\left(\operatorname{nid}_{B_{2} \backslash \overline{B_{1}}}\right)\right]((1 / n) x)$ for $x \in \mathbb{R}^{3}$. Theorem 2.7 and Lemma 2.3 yield that $\mathfrak{D}_{n} \in C_{0}^{\infty}\left(A_{n}\right)^{3}$ and $\operatorname{div}\left(\varphi_{n} W+\mathfrak{D}_{n}\right)=0$. It further follows from Theorem 2.7, in particular its last statement, and from Lemma 2.3 and (5.1) that

$$
\left\|\mathfrak{D}_{n}\right\|_{p} \leq \mathfrak{C}(p)\left\|W\left|A_{n}\left\|_{p}, \quad\right\| \partial^{\alpha} \mathfrak{D}_{n}\left\|_{p} \leq \mathfrak{C}(p) n^{-1}\right\| W\right| A_{n}\right\|_{1, p},
$$

for any $\alpha \in \mathbb{N}_{0}^{3}$ with $1 \leq|\alpha| \leq 2$, and for any $p \in(1, \infty)$. On the other hand, as remarked above, equation (3.5) is valid for $(V, \Pi)$ with $G=0$. Observing that $\varphi_{n} W+\mathfrak{D}_{n} \in$ $C_{0}^{\infty}\left(B_{2 n}\right)^{3}$, and recalling that $\operatorname{div}\left(\varphi_{n} W+\mathfrak{D}_{n}\right)=0$ for $n \in \mathbb{N}$, we may thus deduce from (5.2) by some integrations by parts that $\int_{\mathbb{R}^{3}} \partial_{l} V \cdot \phi d x=\lim _{n \rightarrow \infty} \mathfrak{A}_{n}$, with

$$
\begin{aligned}
\mathfrak{A}_{n}: & =-\int_{\mathbb{R}^{3}} V \cdot\left(2 \sum_{k=1}^{3} \partial_{k} \varphi_{n} \partial_{k} W+\Delta \varphi_{n} W+\mu \tau \partial_{1} \varphi_{n} W+\Delta \mathfrak{D}_{n}+\mu \tau \partial_{1} \mathfrak{D}_{n}\right. \\
& \left.-\lambda \mathfrak{D}_{n}-\Gamma \nabla \varphi_{n}\right) d x \quad \text { for } n \in \mathbb{N} .
\end{aligned}
$$


Since the support of any derivative of $\varphi_{n}$ is a subset of $A_{n}$, and because of (5.1), we get for $n \in \mathbb{N}$ with $n \geq R_{0}:=\max \left\{R_{1}, R_{2}, R_{3}\right\}$ that

$$
\begin{aligned}
\left|\mathfrak{A}_{n}\right| \leq & \mathfrak{C} \sum_{i=1}^{3}\left\|V^{(i)} \mid B_{n}^{c}\right\|_{r_{i}}\left(\left\|\nabla W\left|A_{n}\left\|_{r_{i}^{\prime}}+n^{-1}\right\| W\right| A_{n}\right\|_{r_{i}^{\prime}}+\sum_{\alpha \in \mathbb{N}_{0}^{3}, 1 \leq|\alpha| \leq 2}\left\|\partial^{\alpha} \mathfrak{D}_{n}\right\|_{r_{i}^{\prime}}\right. \\
& \left.+|\lambda|\left\|\mathfrak{D}_{n}\right\|_{r_{i}^{\prime}}+\left\|\Gamma \mid A_{n}\right\|_{r_{i}^{\prime}}\right) .
\end{aligned}
$$

Next we apply (5.3) to obtain

$$
\begin{aligned}
\left|\mathfrak{A}_{n}\right| \leq & \mathfrak{C} \sum_{i=1}^{3}\left\|V^{(i)} \mid B_{n}^{c}\right\|_{r_{i}}\left(\left\|\nabla W\left|A_{n}\left\|_{r_{i}^{\prime}}+n^{-1}\right\| W\right| A_{n}\right\|_{1, r_{i}^{\prime}}\right. \\
& \left.+|\lambda|\left\|W\left|A_{n}\left\|_{r_{i}^{\prime}}+\right\| \Gamma\right| A_{n}\right\|_{r_{i}^{\prime}}\right) \quad\left(n \in \mathbb{N}, n \geq R_{0}\right) .
\end{aligned}
$$

Lemma 2.2 yields that

$$
n^{-1}\left\|W \mid A_{n}\right\|_{r_{i}^{\prime}} \leq \mathfrak{C}\left(\left\|W\left|{\overline{B_{n}}}^{c}\left\|_{3}+\right\| \nabla W\right|{\overline{B_{n}}}^{c}\right\|_{r_{i}^{\prime}}+n^{-1}\|W\|_{3}+n^{-1}\|\nabla W\|_{r_{i}^{\prime}}\right)
$$

for $n \in \mathbb{N}, n \geq R_{0}$. So we may deduce from (5.4) that

$$
\begin{aligned}
\left|\mathfrak{A}_{n}\right| \leq & \mathfrak{C} \sum_{i=1}^{3}\left\|V^{(i)} \mid B_{n}^{c}\right\|_{r_{i}}\left(\left\|W\left|B_{n}^{c}\left\|_{3}+\right\| \nabla W\right| B_{n}^{c}\right\|_{r_{i}^{\prime}}+n^{-1}\|W\|_{3}\right. \\
& \left.+n^{-1}\|\nabla W\|_{r_{i}^{\prime}}+|\lambda|\left\|W\left|A_{n}\left\|_{r_{i}^{\prime}}+\right\| \Gamma\right| B_{n}^{c}\right\|_{r_{i}^{\prime}}\right) \quad\left(n \in \mathbb{N}, n \geq R_{0}\right) .
\end{aligned}
$$

As the reader may recall, we have $W \in L^{p}\left(\mathbb{R}^{3}\right)^{3}$ for $p \in(4 / 3, \infty), \quad \nabla W \in L^{p}\left(\mathbb{R}^{3}\right)^{3}$ for $p>1$. Thus we may conclude that $\left\|\nabla W\left|B_{n}^{c}\left\|_{r_{i}^{\prime}}+\right\| W\right| B_{n}^{c}\right\|_{3} \rightarrow 0$ and $n^{-1}\|W\|_{3}+$ $n^{-1}\|\nabla W\|_{r_{i}^{\prime}} \rightarrow 0(n \rightarrow \infty)$ for $1 \leq i \leq 3$. Similarly, recalling that $\Gamma \in L^{p}\left(\mathbb{R}^{3}\right)$ for $p \in(1, \infty)$, we get $\left\|\Gamma \mid B_{n}^{c}\right\|_{r_{i}^{\prime}} \rightarrow 0(n \rightarrow \infty, 1 \leq i \leq 3)$. Finally, if $\lambda \neq 0$, we have $W \in L^{p}\left(\mathbb{R}^{3}\right)^{3}$ for $p>1$, so $|\lambda|\left\|W \mid A_{n}\right\|_{r_{i}^{\prime}} \rightarrow 0(n \rightarrow \infty, 1 \leq i \leq 3)$. Therefore inequality (5.5) implies that $\left|\mathfrak{A}_{n}\right| \rightarrow 0$. Since $\int_{\mathbb{R}^{3}} \partial V \cdot \phi d x=\lim _{n \rightarrow \infty} \mathfrak{A}_{n}$, as observed above, this means that $\int_{\mathbb{R}^{3}} \partial_{l} V \cdot \phi d x=0$. But the function $\phi$ was arbitrarily chosen in $C_{0}^{\infty}\left(\mathbb{R}^{3}\right)^{3}$, so $\partial_{l} V=0$. This is true for any $l \in\{1,2,3\}$.

Recalling that $V \in C^{\infty}\left(\mathbb{R}^{3}\right)^{3}$, we thus see there is $c \in \mathbb{C}^{3}$ with $V=c$. But for $\epsilon>$ $0, i \in\{1,2,3\}$, the relation $\left|\left\{x \in B_{R_{0}}^{c}:\left|V^{(i)}(x)\right| \geq \epsilon\right\}\right|<\infty$ holds because otherwise the assumption $V^{(i)} \mid B_{R_{i}}^{c} \in L^{r_{i}}\left(B_{R_{i}}^{c}\right)^{3}$ could not be true. Now suppose that $c \neq 0$. Then $\left\{x \in B_{R_{0}}^{c}: V(x)=c\right\} \subset \cup_{i=1}^{3}\left\{x \in B_{R_{0}}^{c}:\left|V^{(i)}(x)\right| \geq|c| / 6\right\}$. On the other hand, the equation $V=c$ means that $\left\{x \in B_{R_{0}}^{c}: V(x)=c\right\}=B_{R_{0}}^{c}$, so there must be a number $i \in\{1,2,3\}$ with $\left|\left\{x \in B_{R_{0}}^{c}:\left|V^{(i)}(x)\right| \geq|c| / 6\right\}\right|=\infty$, a contradiction! Therefore $c=0$, so the theorem is proved.

Now we consider the exterior domain case (weak solutions to problem (1.8)).

Theorem 5.2 Let $\lambda \in K_{\tau}$ and $R_{0} \in[S, \infty)$. For $i \in\{1,2\}$, let $q_{i}, s_{i} \in(1, \infty), V^{(i)} \in$ $W_{\text {loc }}^{1,1}\left(\bar{\Omega}^{c}\right)^{3}$ with $\nabla V^{(i)} \in L^{q_{i}}\left(\bar{\Omega}^{c}\right)^{9}$ (hence $V^{(i)} \mid \Omega_{R} \in W^{1, q_{i}}\left(\Omega_{R}\right)^{3}$ for $R \in[S, \infty)$ by Theorem 2.1), and $V^{(i)} \mid B_{R_{0}}^{c} \in L^{s_{i}}\left(B_{R_{0}}^{c}\right)^{3}$. Further suppose that $\operatorname{div}\left(V^{(1)}-V^{(2)}\right)=0, V^{(1)}-$ $V^{(2)} \mid \partial \Omega=0$, and that (3.1) holds with $A=\bar{\Omega}^{c}, G=0, V=V^{(1)}-V^{(2)}$. Then $V^{(1)}-V^{(2)}=$ 0 . 
Proof: Put $V:=V^{(1)}-V^{(2)}, q:=\min \left\{q_{1}, q_{2}\right\}$. Then $V \in W_{l o c}^{1,1}\left(\bar{\Omega}^{c}\right)^{3}, \nabla V \in L_{l o c}^{q}\left(\bar{\Omega}^{c}\right)^{9}$ (hence $V \mid \Omega_{R} \in W^{1, q}\left(\Omega_{R}\right)^{3}$ for $R \in[S, \infty)$ by Theorem 2.1), $V \mid \partial \Omega=0, V$ is solenoidal, and equation (3.1) holds with $A=\bar{\Omega}^{c}, G=0$. In particular, there is a pressure $\Pi \in$ $L_{l o c}^{q}\left(\bar{\Omega}^{c}\right)$ associated with $V$ (Theorem 3.1). From Corollary 3.4, we may conclude that $V_{j}, \Pi \in C^{\infty}\left(\bar{\Omega}^{c}\right)(1 \leq j \leq 3)$, and equation (3.5) is valid with $G=0$. In addition, Corollary 3.3 yields that

$$
V\left|\Omega_{R} \in W^{2, p}\left(\Omega_{R}\right)^{3}, \Pi\right| \Omega_{R} \in W^{1, p}\left(\Omega_{R}\right)^{3} \text { for } R \in[S, \infty), p \in(1, \infty) .
$$

Fix a function $\varphi \in C^{\infty}\left(\mathbb{R}^{3}\right)$ with $0 \leq \varphi \leq 1, \varphi\left|B_{R_{0}+1 / 4}=0, \varphi\right| B_{R_{0}+3 / 4}^{c}=1$, and abbreviate $B:=B_{R_{0}+1} \backslash \overline{B_{R_{0}}}$. By Theorem 2.1 and our assumptions on $V^{(i)}$, we get

$$
\nabla\left(\varphi V^{(i)}\right) \in L^{q_{i}}\left(\mathbb{R}^{3}\right)^{9}, \varphi V^{(i)}\left|B_{R_{0}+1}^{c}=V^{(i)}\right| B_{R_{0}+1}^{c} \in L^{s_{i}}\left(B_{R_{0}+1}^{c}\right)^{3}(i \in\{1,2\}) .
$$

Since $\nabla \varphi \cdot V \in C_{0}^{\infty}(B), V \mid \partial \Omega=0$ and $\operatorname{div} V=0$, we have $\int_{B} \nabla \varphi \cdot V d x=0$. Thus we may apply Theorem 2.7 , setting $\mathfrak{D}:=\mathfrak{D}\left(2,1, R_{0}, R_{0}+1\right)(-\nabla \varphi \cdot V \mid B)$. Note that $\mathfrak{D} \in C_{0}^{\infty}(B)^{3}$. We further define $\widetilde{V}, \widetilde{\Pi}, \widetilde{F}$ as the zero extension to $\mathbb{R}^{3}$ of $\varphi V+\mathfrak{D}, \varphi \Pi$ and $-2 \sum_{k=1}^{3} \partial_{k} \varphi \partial_{k} V-\Delta \varphi V+\tau \partial_{1} \varphi V-\Delta \mathfrak{D}+\tau \partial_{1} \mathfrak{D}+\lambda \mathfrak{D}+\Pi \nabla \varphi$, respectively. By the choice of $\mathfrak{D}$ and Theorem 2.7, we have $\operatorname{div} \widetilde{V}=0$. Moreover $\widetilde{V}_{j}, \widetilde{\Pi} \in C^{\infty}\left(\mathbb{R}^{3}\right)(1 \leq j \leq$ $3), \widetilde{F} \in C_{0}^{\infty}(B)^{3}$, and the pair $(\widetilde{V}, \widetilde{\Pi})$ satisfies $(3.5)$ with $V, \Pi, G$ replaced by $\widetilde{V}, \widetilde{\Pi}, \widetilde{F}$, respectively. Since $\widetilde{F} \in C_{0}^{\infty}(B)^{3}$, we may apply Corollary 4.3, allowing us to choose functions $W \in C^{\infty}\left(\mathbb{R}^{3}\right)^{3}, \Gamma \in C^{\infty}\left(\mathbb{R}^{3}\right)$ such that the pair $(W, \Gamma)$ solves $(3.5)$ with $V, \Pi, G$ replaced by $W, \Gamma, \widetilde{F}$, respectively, and such that in addition $W \in W^{2,2}\left(\mathbb{R}^{3}\right)^{3}$ in the case $\lambda \neq 0$, and $W \in L^{p}\left(\mathbb{R}^{3}\right)^{3}$ for $p \in(2, \infty), \nabla W \in L^{p}\left(\mathbb{R}^{3}\right)^{9}$ for $p \in(4 / 3, \infty), D^{2} W \in$ $L^{p}\left(\mathbb{R}^{3}\right)^{27}$ for $p \in(1, \infty)$ if $\lambda=0$. In particular $\varphi V^{(1)}-\varphi V^{(2)}-W+\mathfrak{D}=\widetilde{V}-W \in$ $W_{\text {loc }}^{1,1}\left(\mathbb{R}^{3}\right)^{3}, \operatorname{div}(\widetilde{V}-W)=0$, and the function $\widetilde{V}-W$ satisfies $(3.1)$ with $A=\mathbb{R}^{3}, G=$ $0, V=\widetilde{V}-W$. Recalling (5.7) and the relations $\mathfrak{D} \in C_{0}^{\infty}(B), W \in C^{\infty}\left(\mathbb{R}^{3}\right)^{3} \cap L^{p}\left(\mathbb{R}^{3}\right)$ for $p>2(\lambda=0)$ or $p=2(\lambda \neq 0), \nabla W \in L^{2}\left(\mathbb{R}^{3}\right)^{9}$, we see we may apply Theorem 5.1 with $V^{(1)}, V^{(2)}, V^{(3)}$ replaced by $\varphi V^{(1)},-\varphi V^{(2)},-W+\mathfrak{D}$, respectively. This theorem yields that $\varphi V^{(1)}-\varphi V^{(2)}-W+\mathfrak{D}=0$, that is, $\widetilde{V}-W=0$. As a consequence, in view of the equation in (5.7) and the relation $\mathfrak{D} \in C_{0}^{\infty}(B)^{3}$, we have $V\left|B_{R_{0}+1}^{c}=\widetilde{V}\right| B_{R_{0}+1}^{c}=$ $W \mid B_{R_{0}+1}^{c}$. This equation, (5.6) and the integrability properties of $W$ listed above yield that $V \in W^{2,2}\left(\bar{\Omega}^{c}\right)^{3}$ if $\lambda \neq 0$, as well as $V \in L^{p}\left(\bar{\Omega}^{c}\right)^{3}$ for $p \in(2, \infty), \nabla V \in L^{p}\left(\bar{\Omega}^{c}\right)^{9}$ for $p \in(4 / 3, \infty), D^{2} V \in L^{p}\left(\bar{\Omega}^{c}\right)^{27}$ for $p \in(1, \infty)$ if $\lambda=0$.

Now choose $p=2$ in the case $\lambda \neq 0$, and $p=3$ if $\lambda=0$. Then, by the integrability properties of $V$ we have just proved, it follows that $V \in W^{1, p}\left(\bar{\Omega}^{c}\right)^{3}$ and $\nabla V \in L^{p^{\prime}}\left(\bar{\Omega}^{c}\right)^{9}$ Since $V \mid \partial \Omega=0$ by our assumptions, we get $V \in W_{0}^{1, p}\left(\bar{\Omega}^{c}\right)^{3}$. But $\operatorname{div} V=0$ also by our assumptions, so Theorem 2.2 yields there is a sequence $\left(\phi_{n}\right)$ in $C_{0}^{\infty}\left(\bar{\Omega}^{c}\right)^{3}$ with $\operatorname{div} \phi_{n}=0$ for $n \in \mathbb{N}$ and $\left\|V-\phi_{n}\right\|_{1, p} \rightarrow 0$. Using $\overline{\phi_{n}}$ as test function in (3.1), letting $n$ tend to infinity, and taking account of the fact that $G=0$ in (3.1) and $\nabla V \in L^{p^{\prime}}(\bar{\Omega})^{9}$, we arrive at an equation whose real part is given by $\|\nabla V\|_{2}^{2}+\Re \int_{\bar{\Omega}^{c}} \partial_{1} V \cdot \bar{V} d x+\gamma(V)=0$, where $\gamma(V)=0$ if $\lambda=0$, and $\gamma(V):=\Re \lambda\|V\|_{2}^{2}$ else.

Fix some function $\psi \in C_{0}^{\infty}\left(B_{2}\right)$ with $\psi \mid B_{1}=1$, and define $\psi_{n}(x):=\psi\left(n^{-1} x\right)$ for $x \in$ $\mathbb{R}^{3}, n \in \mathbb{N}$. Then $\psi_{n} \in C_{0}^{\infty}\left(B_{2 n}\right), \psi_{n} \mid B_{n}=1$, and $\left|\nabla \psi_{n}\right|_{\infty} \leq \mathfrak{C} n^{-1}$. On the other hand, since $\partial_{1} V \in L^{p^{\prime}}\left(\bar{\Omega}^{c}\right)^{3}, V \in L^{p}\left(\bar{\Omega}^{c}\right)^{3}$ by our choice of $p$, hence $\partial_{1} V \cdot \bar{V} \in L^{1}\left(\bar{\Omega}^{c}\right)^{3}$, we have 
$\int_{\bar{\Omega}^{c}} \partial_{1} V \cdot \bar{V} d x=\lim _{n \rightarrow \infty} \int_{\bar{\Omega}^{c}} \partial_{1} V \cdot \bar{V} \psi_{n} d x$. But $V \in C^{\infty}\left(\mathbb{R}^{3}\right)^{3}$, so

$$
\Re \int_{\bar{\Omega}^{c}} \partial_{1} V \cdot \bar{V} \psi_{n} d x=\int_{\bar{\Omega}^{c}}\left[\partial_{1}(\Re V) \cdot \Re V+\partial_{1}(\Im V) \cdot \Im V\right] \psi_{n} d x=-\int_{\bar{\Omega}^{c}}|V|^{2} \partial_{1} \psi d x / 2 .
$$

But $|V|^{2} \in L^{r}\left(\bar{\Omega}^{c}\right)$ for any $r \in(1, \infty)$, as is immediately clear if $\lambda=0$, and follows from the relation $V \in W^{2,2}\left(\bar{\Omega}^{c}\right)^{3}$ by Sobolev inequalities in the case $\lambda \neq 0$. In addition we have $\left(\int_{\bar{\Omega}^{c}}\left|\partial_{1} \psi_{n}\right|^{s} d x\right)^{1 / s} \leq \mathfrak{C} n^{-1+3 / s}$ for $s \in(1, \infty)$. Therefore $\int \bar{\Omega}^{c}|V|^{2} \partial_{1} \psi d x \rightarrow 0$ for $n \rightarrow \infty$, so we obtain $\|\nabla V\|_{2}^{2}+\gamma(V)=0$. As a consequence $\nabla V=0$. Since $V \in C^{\infty}\left(\bar{\Omega}^{c}\right)^{3}$, we may conclude that $V=0$.

\section{Oseen resolvent estimates.}

We begin by presenting two corollaries where we collect some features of solutions to (1.8) following immediately from the existence results in Theorem 3.6 and 3.7, and from the uniqueness properties stated in Theorem 5.2 and Corollary 2.2. The notation we introduce for these solutions in the two corollaries in question will be used frequently in what follows.

Corollary 6.1 Let $\lambda \in K_{\tau} \backslash\{0\}$. For any $G \in \cup_{q \in(1, \infty)} L^{q}\left(\bar{\Omega}^{c}\right)^{3}$ or $G \in \cup_{q \in(1, \infty)} L^{q}\left(\mathbb{R}^{3}\right)^{3}$, there is a unique function $V:=\mathfrak{V}(\lambda, G) \in \cup_{q \in(1, \infty)} W^{2, q}\left(\bar{\Omega}^{c}\right)^{3}$ and a unique function $\Pi:=\Pi(\lambda, G) \in \cup_{q \in(1, \infty)} W_{l o c}^{1, q}\left(\bar{\Omega}^{c}\right)$ such that $\int_{\Omega_{S+1}} \Pi d x=0$ and the pair $(V, \Pi)$ solves (1.8), with $G \mid \bar{\Omega}^{c}$ instead of $G$ if $G \in \cup_{q \in(1, \infty)} L^{q}\left(\mathbb{R}^{3}\right)^{3}$.

If $p \in(1, \infty)$ and $G \in L^{p}\left(\bar{\Omega}^{c}\right)^{3} \cup L^{p}\left(\mathbb{R}^{3}\right)^{3}$, then $V \in W^{2, p}\left(\bar{\Omega}^{c}\right)^{3}$ and $\nabla \Pi \in L^{p}\left(\bar{\Omega}^{c}\right)^{3}$.

In particular, if $G \in C_{0}^{\infty}\left(\bar{\Omega}^{c}\right)^{3}$, we have $V \in W^{2, p}\left(\bar{\Omega}^{c}\right)^{3}$ and $\nabla \Pi \in L^{p}\left(\bar{\Omega}^{c}\right)^{3}$ for any $p \in(1, \infty)$.

corollaryc7.20〉 Corollary 6.2 For any $G \in \cup_{q \in(1,2)} L^{q}\left(\bar{\Omega}^{c}\right)^{3}$ or $G \in \cup_{q \in(1,2)} L^{q}\left(\mathbb{R}^{3}\right)^{3}$, there is a unique function $V:=\mathfrak{V}(0, G) \in W_{\text {loc }}^{2,1}\left(\bar{\Omega}^{c}\right)^{3}$ and a unique function $\Pi:=\Pi(0, G) \in W_{\text {loc }}^{1,1}\left(\bar{\Omega}^{c}\right)$ such that $\int_{\Omega_{S+1}} \Pi d x=0, \quad V \in \cup_{q \in(2, \infty)} L^{q}\left(\bar{\Omega}^{c}\right)^{3}, \quad \nabla V \in \cup_{q \in(4 / 3, \infty)} L^{q}\left(\bar{\Omega}^{c}\right)^{9}, \quad D^{2} V \in$ $\cup_{q \in(1, \infty)} L^{q}\left(\bar{\Omega}^{c}\right)^{27}, \quad \nabla \Pi \in \cup_{q \in(1, \infty)} L^{q}\left(\bar{\Omega}^{c}\right)^{3}$, and such that (1.8) holds with $\lambda=0$, and with with $G \mid \bar{\Omega}^{c}$ instead of $G$ if $G \in \cup_{q \in(1,2)} L^{q}\left(\mathbb{R}^{3}\right)^{3}$.

If $p \in(1,2)$ and $G \in L^{p}\left(\bar{\Omega}^{c}\right)^{3} \cup L^{p}\left(\mathbb{R}^{3}\right)^{3}$, then the relations $V \in L^{(1 / p-1 / 2)^{-1}}\left(\bar{\Omega}^{c}\right)^{3}, \nabla V \in$ $L^{(1 / p-1 / 4)^{-1}}\left(\bar{\Omega}^{c}\right)^{9}, D^{2} V \in L^{p}\left(\bar{\Omega}^{c}\right)^{27}, \nabla \Pi \in L^{p}\left(\bar{\Omega}^{c}\right)^{3}$, hold, in particular $V \mid \Omega_{R} \in W^{2, p}\left(\Omega_{R}\right)^{3}$ for $R \in[S, \infty)$.

Moreover, if $G \in C_{0}^{\infty}\left(\bar{\Omega}^{c}\right)^{3}$, then $V \in L^{p}\left(\bar{\Omega}^{c}\right)^{3}$ for $p \in(2, \infty), \nabla V \in L^{p}\left(\bar{\Omega}^{c}\right)^{9}$ for $p \in$ $(4 / 3, \infty), D^{2} V \in L^{p}\left(\bar{\Omega}^{c}\right)^{27}$ and $\nabla \Pi \in L^{p}\left(\bar{\Omega}^{c}\right)^{3}$ for $p \in(1, \infty)$.

The inequalities stated in the next theorem are preliminary versions of our resolvent estimates. In these first versions, the unknowns still appear on the right-hand side.

Corollary 6.3 Let $\lambda \in K_{\tau}$ and $G \in C_{0}^{\infty}\left(\bar{\Omega}^{c}\right)^{3}$. Put $V:=\mathfrak{V}(\lambda, G), \Pi:=\Pi(\lambda, G)$. Then $V \in C^{\infty}\left(\bar{\Omega}^{c}\right)^{3}$ and $\Pi \in C^{\infty}\left(\bar{\Omega}^{c}\right)$.

Abbreviate $\mathfrak{K}_{q}(G, V, \Pi):=\|G\|_{q}+\left\|\nabla V\left|\Omega_{S+1}\left\|_{q}+\right\| \Pi\right| \Omega_{S+1}\right\|_{q}$ for $q \in(1, \infty)$. 
Then $\|V\|_{p} \leq \mathfrak{C}(p, q) \mathfrak{K}_{q}(G, V, \Pi)$ for $q \in(1,2), p \in\left((1 / q-1 / 2)^{-1}, \infty\right)$ if $q \geq 3 / 2, p \in$ $\left((1 / q-1 / 2)^{-1},(1 / q-2 / 3)^{-1}\right)$ if $q<3 / 2$, and for $(p, q)=(6,6 / 5)$.

Moreover $\|\nabla V\|_{p} \leq \mathfrak{C}(p, q) \mathfrak{K}_{q}(G, V, \Pi)$ for $q \in(1,3], p \in\left((1 / q-1 / 4)^{-1},(1 / q-1 / 3)^{-1}\right)$, and for $(p, q)=(2,6 / 5)$.

Furthermore $\left\|D^{2} V \mid \Omega_{R}\right\|_{q} \leq \mathfrak{C}(q, R) \mathfrak{K}_{q}(G, V, \Pi)$ for $q \in(1, \infty), \quad R \in[S, \infty),\left\|D^{2} V\right\|_{2} \leq$ $\mathfrak{C} \mathfrak{K}_{2}(G, V, \Pi),\|\nabla \Pi\|_{q} \leq \mathfrak{C}(q) \mathfrak{K}_{q}(G, V, \Pi)$ for $q \in(1, \infty)$

In addition, if $\lambda \neq 0$, the inequality $\|V\|_{p} \leq \mathfrak{C}(p, q)|\lambda|^{-2+4(1 / q-1 / p)} \mathfrak{K}_{q}(G, V, \Pi)$ holds for $q \in(1,2], p \in[q, 2]$.

There is $c_{0} \in \mathbb{C}$ such that $\Pi+c_{0} \in L^{p}\left(\bar{\Omega}^{c}\right)$ for $p \in(3 / 2, \infty)$. Put $B:=B_{S+1} \backslash \overline{B_{S}}$. Then for $p \in(4,6)$,

$$
\|V\|_{p}+\|\nabla V\|_{2}+\left\|\Pi+c_{0}\right\|_{2} \leq \mathfrak{C}(p)\left(\left\|G\left|\bar{\Omega}^{c}\left\|_{-1,2}+\right\| V\right| B\right\|_{2}+\|\Pi \mid B\|_{-1,2}\right) .
$$

Proof: By Corollary 3.4, we have $V \in C^{\infty}\left(\bar{\Omega}^{c}\right)^{3}$ and $\Pi \in C^{\infty}\left(\bar{\Omega}^{c}\right)$. Moreover Corollary 3.3 yields

$$
V\left|\Omega_{R} \in W^{2, p}\left(\Omega_{R}\right)^{3}, \Pi\right| \Omega_{R} \in W^{1, p}\left(\Omega_{R}\right) \quad \text { for } p \in(1, \infty), R \in[S, \infty) .
$$

Recall that $B=B_{S+1} \backslash \overline{B_{S}}$. Choose some function $\varphi \in C_{0}^{\infty}\left(\mathbb{R}^{3}\right)$ satisfying the relations $0 \leq \varphi \leq 1, \varphi\left|B_{S+1 / 4}=1, \varphi\right| B_{S+3 / 4}^{c}=0$. Then $\nabla \varphi \cdot V \in C_{0}^{\infty}(B)$. Recalling that the pair $(V, \Pi)$ is a solution to $(1.8)$, we note that $V \mid \partial \Omega=0$ and $\operatorname{div} V=0$, so $\int_{B} \nabla \cdot V d x=0$. Therefore we may apply Theorem 2.7 , setting $\mathfrak{D}:=\mathfrak{D}(2,1, S, S+1)(\nabla \varphi \cdot V \mid B)$. This means in particular that $\mathfrak{D} \in C_{0}^{\infty}(B)^{3}$. Moreover, taking account of the last statement of Theorem 2.7, we get

$$
\|\mathfrak{D}\|_{2, p} \leq C(p)\|\nabla \varphi \cdot V\|_{1, p} \leq C(p)\|V \mid B\|_{1, p} \quad \text { for } p \in(1, \infty) .
$$

Let $\widetilde{V}, \widetilde{\Pi}, H$ denote the zero extension to $\mathbb{R}^{3}$ of $(1-\varphi) V+\mathfrak{D},(1-\varphi) \Pi$ and $2 \sum_{k=1}^{3} \partial_{k} \varphi \partial_{k} V$ $+\Delta \varphi V-\tau \partial_{1} \varphi V-\Delta \mathfrak{D}+\tau \partial_{1} \mathfrak{D}+\lambda \mathfrak{D}-\Pi \nabla \varphi$, respectively. Note that $H \in C_{0}^{\infty}(B)^{3}$. By Theorem 2.7 and the choice of $\mathfrak{D}$, we have $\operatorname{div} \widetilde{V}=0$. Thus the pair $(\widetilde{V}, \widetilde{\Pi})$ solves $(3.5)$ with $V, \Pi, G$ replaced by $\widetilde{V}, \widetilde{\Pi}$ and $(1-\varphi) G+H$, respectively. In view of Corollary 6.1 and 6.2 , we know that $V \in L^{p}\left(\bar{\Omega}^{c}\right)^{3}$ and $\nabla V \in L^{r}\left(\bar{\Omega}^{c}\right)^{9}$ for certain $p, r \in(1, \infty)$. Therefore and because of the relations $\mathfrak{D} \in C_{0}^{\infty}(B)^{3}, V_{j}, \Pi \in C^{\infty}\left(\bar{\Omega}^{c}\right)$ for $1 \leq j \leq 3$, an analogous property is true for $\widetilde{V}$ and $\nabla \widetilde{V}$, respectively. On the other hand, since $(1-\varphi) G+H \in C_{0}^{\infty}\left(\mathbb{R}^{3}\right)^{3}$, the functions $\bar{V}:=E^{(\lambda)} * P_{2}((1-\varphi) G+H), \bar{\Pi}:=Q_{2}((1-\varphi) G+H)$ satisfy all the properties listed in the first part of Corollary 4.3, with $\bar{V}, \bar{\Pi},(1-\varphi) G+H$ in the place of $V, \Pi, G$, respectively. Thus we may apply Theorem 5.1 to obtain $\widetilde{V}=\bar{V}$. This implies there is $c_{0} \in \mathbb{C}$ with $\widetilde{\Pi}+c_{0}=\bar{\Pi}$. In view of the choice of $\varphi$, and because $\mathfrak{D} \in C_{0}^{\infty}(B)^{3}$, we may conclude that

$$
V\left|B_{S+1}^{c}=\widetilde{V}\right| B_{S+1}^{c}=\bar{V}\left|B_{S+1}^{c}, \quad \Pi+c_{0}\right| B_{S+1}^{c}=\widetilde{\Pi}+c_{0}\left|B_{S+1}^{c}=\bar{\Pi}\right| B_{S+1}^{c} .
$$

From (6.2), (6.4) and the fact that $\bar{\Pi}$ possesses all the properties stated for $\Pi$ in Corollary 4.3, we may conclude that $\Pi+c_{0} \in L^{p}\left(\bar{\Omega}^{c}\right)$ for $p \in(3 / 2, \infty)$, as claimed in Corollary 6.3. In view of (6.3), we get

$$
\|H\|_{p} \leq \mathfrak{C}(p)\left(\left\|V\left|B\left\|_{1, p}+\right\| \Pi\right| B\right\|_{p}\right) \quad \text { for } p \in(1, \infty) .
$$


Since $V \mid \partial \Omega=0$, Poincaré's inequality applied on $\Omega_{S+1}$ yields

$$
\|G\|_{p}+\left\|V\left|B\left\|_{1, p}+\right\| \Pi\right| B\right\|_{p} \leq \mathfrak{C}(p) \mathfrak{K}_{p}(G, V, \Pi) \text { for } p \in(1, \infty) .
$$

At this point we observe that the functions $\bar{V}$ and $\bar{\Pi}$ may be estimated by applying Corollary 4.1 with $G$ replaced by $(1-\varphi) G+H$, and then referring to (6.5) and (6.6). Taking account of the equations $\bar{V}=\widetilde{V}$ and $\bar{\Pi}=\widetilde{\Pi}+c_{0}$, we thus see that the inequalities in Corollary 6.3 up to but excluding (6.1), with $\widetilde{V}, \widetilde{\Pi}$ in the role of $V$ and $\Pi$, respectively, follow from Corollary 4.1.

Put $W:=\varphi V-\mathfrak{D}, \quad \Gamma:=\varphi \Pi$. Then $W \in C^{\infty}\left(\bar{\Omega}^{c}\right)^{3}, \quad \Gamma \in C^{\infty}\left(\bar{\Omega}^{c}\right)$, and the equations in (3.5) hold with $V, \Pi, G$ replaced by $W, \Gamma, \varphi G-H$, respectively. From (6.2), we conclude that $W \mid \Omega_{S+1} \in W^{2, q}\left(\Omega_{S+1}\right)^{3}$ and $\Pi \mid \Omega_{S+1} \in W^{1, q}\left(\Omega_{S+1}\right)$ for any $q \in(1, \infty)$. Moreover, since $\mathfrak{D} \in C_{0}^{\infty}(B)^{3}$ and by the choice of $\varphi$ and $G$, we have $\varphi G-H \in$ $C_{0}^{\infty}\left(\Omega_{S+1}\right)^{3}, W\left|B_{S+3 / 4}^{c}=0, \Gamma\right| B_{S+3 / 4}^{c}=0$ and $W|\partial \Omega=V| \partial \Omega=0$. This means in particular that $W \mid \Omega_{S+1} \in W_{0}^{1, q}\left(\Omega_{S+1}\right)^{3}$ for any $q \in(1, \infty)$.

Thus we see that we may apply Theorem 3.5, in particular the estimate at the end of this theorem, as well as Corollary 3.6 with $A=\Omega_{S+1}$ and with $V, \Pi, G$ replaced by $W\left|\Omega_{S+1}, \Gamma\right| \Omega_{S+1}, \varphi G-H \mid \Omega_{S+1}$, respectively. Again taking account of (6.5) and (6.6), and recalling that $W$ and $\Gamma$ vanish outside $B_{S+1}$, we conclude that the first, second and sixth (if $\lambda \neq 0$ ) inequality in Corollary 6.3 with $V$ replaced by $W$ on the left-hand side follow from Corollary 3.6. Inequalities (6.5) and (6.6) and the estimate at the end of Theorem 3.5 yield that $\left\|D^{2} W\right\|_{q}+\|\nabla \Pi\|_{q}$ is bounded by $\mathfrak{C}(q) \mathfrak{K}_{q}(G, V, \Pi)$ for $q \in(1, \infty)$. This means that the third, forth and fifth estimate in Corollary 6.3 hold with $V$, $\Pi$ replaced by $W, \Gamma$, respectively, on the left-hand side. Since $V=\widetilde{V}+W, \Pi=\widetilde{\Pi}+\Gamma$, we have thus proved the estimates up to but excluding (6.1) in Corollary 6.3.

In order to derive (6.1), take $\gamma \in C_{0}^{\infty}\left(\mathbb{R}^{3}\right)^{3}$. With (2.1), we get $\left\|\gamma\left|B\left\|_{2} \leq C(S)\right\| \gamma\right| B\right\|_{6} \leq$ $C(S)\left\|\gamma \mid{\overline{B_{S}}}^{c}\right\|_{6} \leq C(S)\|\nabla \gamma\|_{2}$. Moreover, by Theorem 2.7, in particular by its last statement, we obtain $\|\mathfrak{D}\|_{1,2} \leq \mathfrak{C}\|\nabla \varphi \cdot V\|_{2} \leq\|V \mid B\|_{2}$. Using these inequalities, as well as the relations $\mathfrak{D} \in C_{0}^{\infty}(B)^{3}$ and $\operatorname{supp}(\nabla \varphi) \subset B$, we get

$$
\begin{aligned}
& \left|\int_{\mathbb{R}^{3}} \Delta \mathfrak{D} \cdot \gamma d x\right| \leq \mathfrak{C}\|\nabla \mathfrak{D}\|_{2}\|\nabla \gamma\|_{2} \leq \mathfrak{C}\|V \mid B\|_{2}\|\nabla \gamma\|_{2}, \\
& \left|\lambda \int_{\mathbb{R}^{3}} \mathfrak{D} \cdot \gamma d x\right| \leq \mathfrak{C}\|\mathfrak{D}\|_{2}\left\|\gamma\left|B\left\|_{2} \leq \mathfrak{C}\right\| V\right| B\right\|_{2}\|\nabla \gamma\|_{2}, \\
& \left|\int_{\mathbb{R}^{3}} \sum_{k=1}^{3} \partial_{k} \varphi \partial_{k} V \cdot \gamma d x\right|=\left|\int_{\mathbb{R}^{3}}\left(\Delta \varphi \gamma-\sum_{k=1}^{3} \partial_{k} \varphi \partial_{k} \gamma\right) \cdot V d x\right| \leq \mathfrak{C}\|V \mid B\|_{2}\|\nabla \gamma\|_{2}, \\
& \left|\int_{\mathbb{R}^{3}} \Pi \nabla \varphi \cdot \gamma d x\right| \leq\left\|\Pi\left|B\left\|_{-1,2}\right\| \nabla \varphi \cdot \gamma\left\|_{1,2} \leq \mathfrak{C}\right\| \Pi\right| B\right\|_{-1,2}\|\nabla \gamma\|_{2} .
\end{aligned}
$$

It is obvious how to handle the remaining terms of the integral $\int_{\mathbb{R}^{3}} H \cdot \gamma d x$. Thus, collecting the preceding estimates, we arrive at the inequality $\|H\|_{-1,2} \leq \mathfrak{C}\left(\left\|V\left|B\left\|_{2}+\right\| \Pi\right| B\right\|_{-1,2}\right)$. Noting that $\operatorname{supp}(1-\varphi) \subset{\overline{B_{S+1 / 4}}}^{c}$ and $\bar{\Omega} \subset B_{S}$, we obtain $(1-\varphi) \gamma \in C_{0}^{\infty}\left(\bar{\Omega}^{c}\right)^{3}$ for $\gamma \in C_{0}^{\infty}\left(\mathbb{R}^{3}\right)^{3}$. On recalling that $\operatorname{supp}(\nabla \varphi) \subset B$ and $\|\gamma \mid B\|_{2} \leq \mathfrak{C}\|\nabla \gamma\|_{2}$, we further find 
that $\|\nabla((1-\varphi) \gamma)\|_{2} \leq \mathfrak{C}\|\nabla \gamma\|_{2}$ for $\gamma \in C_{0}^{\infty}\left(\mathbb{R}^{3}\right)^{3}$. As a consequence $\|(1-\varphi) G\|_{-1,2} \leq$ $\mathfrak{C}\left\|G \mid \bar{\Omega}^{c}\right\|_{-1,2}$. In view of the estimate of $\|H\|_{-1,2}$ given above, this means

$$
\|(1-\varphi) G+H\|_{-1,2} \leq \mathfrak{C}\left(\left\|G\left|\bar{\Omega}^{c}\left\|_{-1,2}+\right\| V\right| B\right\|_{2}+\|\Pi \mid B\|_{-1,2}\right) .
$$

Next we remark that the functions $\bar{V}$ and $\bar{\Pi}$ may be estimated by making use of Theorem 4.3 and Lemma 4.1 with $G$ replaced by $(1-\varphi) G+H$. Again recalling that $\bar{V}=\widetilde{V}$ and $\bar{\Pi}=\widetilde{\Pi}+c_{0}$, we may then conclude that inequality (6.1) with $V$, $\Pi$ replaced by $\widetilde{V}, \widetilde{\Pi}$, respectively, follows from Theorem 4.3, Lemma 4.1 and (6.7).

In order to estimate $W$ and $\Gamma$, we fix a function $\zeta \in C_{0}^{\infty}(B)$ with $\zeta \geq 0$ and $\int_{B} \zeta d x>0$. Put $c_{\zeta}:=\left(\int_{B} \zeta d x\right)^{-1} \int_{B} \Gamma \zeta d x$. Corollary 3.5 with $A=\Omega_{S+1}$ and with $G$ replaced by $\varphi G-H \mid \Omega_{S+1}$ imply that

$$
\|W\|_{1,2}+\|W\|_{p}+\left\|\Gamma-c_{\zeta}\left|\Omega_{S+1}\left\|_{2} \leq \mathfrak{C}(p)\right\| \varphi G-H\right| \Omega_{S+1}\right\|_{-1,2} \quad \text { for } p \in(4,6) .
$$

Obviously $\left\|\varphi G-H\left|\Omega_{S+1}\left\|_{-1,2} \leq\right\| \varphi G-H\right| \bar{\Omega}^{c}\right\|_{-1,2} \leq\left\|\varphi G \mid \bar{\Omega}^{c}\right\|_{-1,2}+\|H\|_{-1,2}$, so we may conclude with the estimate of $\|H\|_{-1,2}$ we derived above that for $p \in(4,6)$,

$$
\|W\|_{1,2}+\|W\|_{p}+\left\|\Gamma-c_{\zeta} \mid \Omega_{S+1}\right\|_{2} \leq \mathfrak{C}(p)\left(\left\|G\left|\bar{\Omega}^{c}\left\|_{-1,2}+\right\| V\right| B\right\|_{2}+\|\Pi \mid B\|_{-1,2}\right) .
$$

Since $V=\widetilde{V}+W, \Pi=\widetilde{\Pi}+\Gamma$, we arrive at inequality (6.1), but with the term $\mathfrak{C} \cdot\left\|c_{\zeta} \mid \Omega_{S+1}\right\|_{2}$ added on the right-hand side. However, since $\zeta \in C_{0}^{\infty}(B)$, hence $\varphi \zeta \in C_{0}^{\infty}(B)$, we get with Poincaré's inequality on $B$ and the definition of $\Gamma$ that

$$
\left\|c_{\zeta}\left|\Omega_{S+1}\left\|_{2} \leq \mathfrak{C}\left|c_{\zeta}\right| \leq \mathfrak{C}\left|\int_{B} \Pi \varphi \zeta d x\right| \leq \mathfrak{C}\right\| \Pi\right| B\right\|_{-1,2}\|\varphi \zeta\|_{1,2} \leq \mathfrak{C}\|\Pi \mid B\|_{-1,2} .
$$

In the last inequality, we subsumed the term $\|\varphi \zeta\|_{1,2}$ in the constant $\mathfrak{C}$. It is in view of (6.8) that we introduced the condition $\int_{A} \pi \zeta d x=0$ in Corollary 3.5. Estimate (6.8) completes the proof of (6.1).

Now we may prove our main results, beginning with

Theorem 6.1 Let $\lambda \in K_{\tau}, q \in(1,2), G \in L^{q}\left(\bar{\Omega}^{c}\right)^{3}$.

Then $\|\mathfrak{V}(\lambda, G)\|_{p_{1}} \leq \mathfrak{C}\left(p_{1}, q\right)\|G\|_{q}$ for $p_{1} \in\left((1 / q-1 / 2)^{-1}, \infty\right)$ if $q \geq 3 / 2, p_{1} \in((1 / q-$ $\left.1 / 2)^{-1},(1 / q-2 / 3)^{-1}\right)$ in the case $q<3 / 2$, and for $p_{1}=6$ if $q=6 / 5$.

Moreover $\|\nabla \mathfrak{V}(\lambda, G)\|_{p_{2}} \leq \mathfrak{C}\left(p_{2}, q\right)\|G\|_{q}$ for $p_{2} \in\left((1 / q-1 / 4)^{-1},(1 / q-1 / 3)^{-1}\right)$, and for $p_{2}=2$ if $q=6 / 5$.

In addition, if $\lambda \neq 0$, we have $\|\mathfrak{V}(\lambda, G)\|_{p_{3}} \leq \mathfrak{C}\left({ }_{3}, q\right)|\lambda|^{-2+4\left(1 / q-1 / p_{3}\right)}\|G\|_{q}$ for $p_{3} \in[q, 2]$.

Finally $\left\|D^{2} \mathfrak{V}(\lambda, G) \mid \Omega_{R}\right\|_{q} \leq \mathfrak{C}(q, R)\|G\|_{q}$ for $q \in(1, \infty), R \in[S, \infty)$, and $\|\nabla \Pi(\lambda, G)\|_{q} \leq$ $\mathfrak{C}(q)\|G\|_{q}$ for $q \in(1, \infty)$.

Proof: We proceed by contradiction, similarly to the approach in [20] and [3]. Take $p_{1}, p_{2}, p_{3}$ as in the theorem, and let $R \in[S+1, \infty)$. Abbreviate $\kappa:=2-4\left(1 / q-1 / p_{3}\right)$. Suppose there is no constant $C_{0}>0$ such that

$$
\begin{gathered}
\|\mathfrak{V}(\varrho, \phi)\|_{p_{1}}+\|\nabla \mathfrak{V}(\varrho, \phi)\|_{p_{2}}+\sup _{T>0}|\varrho|^{\kappa}\left\|\chi_{B_{T}} \mathfrak{V}(\varrho, \phi)\right\|_{p_{3}}+\left\|D^{2} \mathfrak{V}(\varrho, \phi) \mid \Omega_{R}\right\|_{q} \\
+\|\nabla \Pi(\varrho, \phi)\|_{q} \leq C_{0}\|\phi\|_{q} \quad \text { for } \phi \in C_{0}^{\infty}\left(\bar{\Omega}^{c}\right)^{3}, \varrho \in K_{\tau} .
\end{gathered}
$$


Then, for any $n \in \mathbb{N}$, there is some $\varrho_{n} \in K_{\tau}$ and some function $\phi_{n} \in C_{0}^{\infty}\left(\bar{\Omega}^{c}\right)^{3}$ with $\gamma_{n} \geq$ $n\left\|\phi_{n}\right\|_{q}$, where $\gamma_{n}:=\left\|\mathfrak{V}\left(\varrho_{n}, \phi_{n}\right)\right\|_{p_{1}}+\left\|\nabla \mathfrak{V}\left(\varrho_{n}, \phi_{n}\right)\right\|_{p_{2}}+\sup _{T>0}\left|\varrho_{n}\right|^{\kappa}\left\|\chi_{B_{T}} \mathfrak{V}\left(\varrho_{n}, \phi_{n}\right)\right\|_{p_{3}}+$ $\left\|D^{2} \mathfrak{V}\left(\varrho_{n}, \phi_{n}\right) \mid \Omega_{R}\right\|_{q}+\left\|\nabla \Pi\left(\varrho_{n}, \phi_{n}\right)\right\|_{q}$. Note that by the regularity properties listed in Corollary 6.1 $\left(\varrho_{n} \neq 0\right)$ or $(6.2)\left(\varrho_{n}=0\right)$, we have $\gamma_{n}<\infty$ for $n \in \mathbb{N}$. This is true even if $\varrho_{n}=0$ for some $n \in \mathbb{N}$, in which case the function $\mathfrak{V}\left(\varrho_{n}, \phi_{n}\right)$ need not belong to $L^{p_{3}}\left(\bar{\Omega}^{c}\right)^{3}$. However the term $\sup _{T>0}\left|\varrho_{n}\right|^{\kappa}\left\|\chi_{B_{T}} \mathfrak{V}\left(\varrho_{n}, \phi_{n}\right)\right\|_{p_{3}}$ then vanishes. We do not use the expression $\left|\varrho_{n}\right|^{\kappa}\left\|\mathfrak{V}\left(\varrho_{n}, \phi_{n}\right)\right\|_{p_{3}}$ for $\varrho \in K_{\tau}, \phi \in C_{0}^{\infty}\left(\bar{\Omega}^{c}\right)^{3}$ because in the case $\varrho=0$, it might lead to products of the form $0 \cdot \infty$, which we want to avoid. Define $v_{n}:=\gamma_{n}^{-1} \mathfrak{V}\left(\varrho_{n}, \phi_{n}\right), \sigma_{n}:=\gamma_{n}^{-1} \Pi\left(\varrho_{n}, \phi_{n}\right), g_{n}:=\gamma_{n}^{-1} \phi_{n}$ for $n \in \mathbb{N}$. Then Corollary $6.1-$ 6.3 and 3.4 yield $v_{n} \in C^{\infty}\left(\bar{\Omega}^{c}\right)^{3}, \sigma_{n} \in C^{\infty}\left(\bar{\Omega}^{c}\right)^{3}, v_{n} \mid \Omega_{R} \in W^{2, q}\left(\Omega_{R}\right)^{3}$,

$$
-\Delta v_{n}+\tau \partial_{1} v_{n}+\varrho_{n} v_{n}+\nabla \sigma_{n}=g_{n}, \quad \operatorname{div} v_{n}=0, \quad v_{n} \mid \partial \Omega=0 \quad \text { for } n \in \mathbb{N} .
$$

Since $\gamma_{n} \geq n\left\|\phi_{n}\right\|_{q}$, we further get

$$
\left\|v_{n}\right\|_{p_{1}}+\left\|\nabla v_{n}\right\|_{p_{2}}+\sup _{T>0}\left|\varrho_{n}\right|^{\kappa}\left\|\chi_{B_{T}} v_{n}\right\|_{p_{3}}+\left\|D^{2} v_{n} \mid \Omega_{R}\right\|_{q}+\left\|\nabla \sigma_{n}\right\|_{q}=1 \geq n\left\|g_{n}\right\|_{q}(6.11) \text { T7.10.80 }
$$

for $n \in \mathbb{N}$. As a first consequence of (6.11), we note that $\left\|g_{n}\right\|_{q} \rightarrow 0$. By the choice of $\Pi\left(\varrho_{n}, \phi_{n}\right)$ (Corollary 6.1 and 6.2), we have $\int_{\Omega_{S+1}} \sigma_{n} d x=0$, hence by a Poincaré's inequality $\left\|\sigma_{n}\left|\Omega_{S+1}\left\|_{q} \leq \mathfrak{C}(q)\right\| \nabla \sigma_{n}\right| \Omega_{S+1}\right\|_{q} \leq \mathfrak{C}(q)\left\|\nabla \sigma_{n}\right\|_{q}$ for $n \in \mathbb{N}$. Thus we may conclude from (6.11) that the sequence $\left(\sigma_{n} \mid \Omega_{S+1}\right)_{n \geq 1}$ is bounded in $W^{1, q}\left(\Omega_{S+1}\right)$. Since $p_{1} \geq q, p_{2} \geq q, R \geq S+1$, it further follows from (6.11) that the sequence $\left(v_{n} \mid \Omega_{S+1}\right)_{n \geq 1}$ is bounded in $W^{2, q}\left(\Omega_{S+1}\right)^{3}$. We may conclude from (6.11) and from the preceding remarks on $\left(\sigma_{n} \mid \Omega_{S+1}\right)_{n \geq 1}$ and $\left.\left(v_{n} \mid \Omega_{S+1}\right)_{n \geq 1}\right)$ that there is a subsequence of $\left(\left(v_{n}, \sigma_{n}, \varrho_{n}\right)\right)$, also denoted by $\left(\left(v_{n}, \sigma_{n}, \varrho_{n}\right)\right)$, with the following properties: $v_{n} \rightarrow V$ in $L^{p_{1}}\left(\bar{\Omega}^{c}\right)^{3}$ for some $V \in L^{p_{1}}\left(\bar{\Omega}^{c}\right)^{3}, \partial_{l} v_{n} \rightarrow V^{(l)}$ in $L^{p_{2}}\left(\bar{\Omega}^{c}\right)^{3}$ for some $V^{(l)} \in L^{p_{2}}\left(\bar{\Omega}^{c}\right)^{3}(1 \leq l \leq 3), \nabla \sigma_{n} \rightarrow \gamma$ in $L^{q}\left(\bar{\Omega}^{c}\right)^{3}$ for some $\gamma \in L^{q}\left(\bar{\Omega}^{c}\right)^{3},\left\|v_{n} \mid \Omega_{S+1}-Z\right\|_{1, q} \rightarrow 0$ for some $Z \in W^{1, q}\left(\Omega_{S+1}\right)^{3}$, $\left\|\sigma_{n} \mid \Omega_{S+1}-Y\right\|_{q} \rightarrow 0$ for some $Y \in L^{q}\left(\Omega_{S+1}\right)^{3}$, and $\varrho_{n} \rightarrow \varrho$ in $\mathbb{C}$ for some $\varrho \in K_{\tau}$.

By considering the sequences $\left(\int_{\bar{\Omega}^{c}} v_{n} \cdot \partial_{l} \psi d x\right)$ and $\left(\int_{\bar{\Omega}^{c}} \partial_{l} v_{n} \cdot \psi d x\right)$ with $l \in\{1,2,3\}, \psi \in$ $C_{0}^{\infty}\left(\bar{\Omega}^{c}\right)^{3}$, we find that $V \in W_{l o c}^{1,1}\left(\bar{\Omega}^{c}\right)^{3}$ and $\partial_{l} V=V^{(l)}$ for $1 \leq l \leq 3$. Similarly we get $V\left|\Omega_{S+1}=Z, Y \in W_{l o c}^{1,1}\left(\Omega_{S+1}\right), \nabla Y=\gamma\right| \Omega_{S+1}$. Since $\partial_{l} V=V^{(l)}(1 \leq l \leq 3)$, we conclude that $\nabla V \in L^{p_{2}}\left(\bar{\Omega}^{c}\right)^{9}$. The equation $\int_{\Omega_{S+1}} \sigma_{n} d x=0$ for $n \in \mathbb{N}$ and the fact that $\left\|\sigma_{n} \mid \Omega_{S+1}-Y\right\|_{q} \rightarrow 0$ yield $\int_{\Omega_{S+1}} Y d x=0$. We further conclude that $\left\|v_{n}-V \mid \Omega_{S+1}\right\|_{1, q} \rightarrow 0$, so $V \mid \partial \Omega=0$ by (6.10). The latter reference, the relation $\left\|g_{n}\right\|_{q} \rightarrow 0$ and the equation $\partial_{l} V=V^{(l)}(1 \leq l \leq 3)$ imply that $\operatorname{div} V=0$ and that (3.1) as well as (3.2) hold with $A=\bar{\Omega}^{c}, G=0, \lambda=\varrho$, and with the term $-\Pi \operatorname{div} \psi$ in (3.2) replaced by $\gamma \cdot \psi$. Recalling that $V \in L^{p_{1}}\left(\bar{\Omega}^{c}\right)^{3}, \nabla V \in L^{p_{2}}\left(\bar{\Omega}^{c}\right)^{9}$, we may now apply Theorem 5.2 to obtain $V=0$. Thus (3.2) reduces to the equation $\int \bar{\Omega}^{c} \gamma \cdot \psi d x=0$ for $\psi \in C_{0}^{\infty}\left(\bar{\Omega}^{c}\right)^{3}$, which means that $\gamma=0$. Since $\nabla Y=\gamma \mid \Omega_{S+1}$ and $\int_{\Omega_{S+1}} Y d x=0$, as mentioned above, we get $Y=0$ (Corollary 2.2). In this way we arrive at the relations $\left\|v_{n} \mid \Omega_{S+1}\right\|_{1, q} \rightarrow 0$ and $\left\|\sigma_{n} \mid \Omega_{S+1}\right\|_{q} \rightarrow 0$.

On the other hand, referring to Corollary 6.3 with $V$, $\Pi$ replaced by $v_{n}=\mathfrak{V}\left(\varrho_{n}, \gamma_{n}^{-1} \phi_{n}\right)$ and $\sigma_{n}=\Pi\left(\varrho_{n}, \gamma_{n}^{-1} \phi_{n}\right)$, respectively, we see that the left-hand side of (6.11) is bounded by a constant times $\left\|g_{n}\right\|_{q}+\left\|v_{n}\left|\Omega_{S+1}\left\|_{1, q}+\right\| \sigma_{n}\right| \Omega_{S+1}\right\|_{q}(n \in \mathbb{N})$. But by what we have found before, this latter term tends to zero for $n \rightarrow \infty$. Thus the left-hand side of (6.11) must equally tend to zero, which is a contradiction to (6.11). So we have shown there is $C_{0}>0$ such that inequality (6.9) holds for $\phi \in C_{0}^{\infty}\left(\bar{\Omega}^{c}\right)^{3}$ and $\varrho \in K_{\tau}$. 
In order to extend this result to $G \in L^{q}\left(\bar{\Omega}^{c}\right)^{3}$, fix some such function $G$, and let $\left(\phi_{n}\right)$ be a sequence in $C_{0}^{\infty}\left(\bar{\Omega}^{c}\right)^{3}$ with $\left\|G-\phi_{n}\right\|_{q} \rightarrow 0$. Let $\varrho \in K_{\tau}$. If $\varrho \neq 0$, then the estimate in Theorem 3.7 yields $\left\|V-V_{n}\right\|_{2, q} \rightarrow 0$ and $\left\|\nabla \Pi-\nabla \Pi_{n}\right\|_{q} \rightarrow 0$, where we used the abbreviations $V=\mathfrak{V}(\varrho, G), \Pi=\Pi(\varrho, G), V_{n}=\mathfrak{V}\left(\varrho, \phi_{n}\right)$ and $\Pi_{n}=\Pi\left(\varrho_{n}, \phi_{n}\right)$, for $n \in \mathbb{N}$. If $\varrho=0$, we use Theorem 3.6 to obtain $\left\|V-V_{n}\right\|_{2 q /(2-q)} \rightarrow 0,\left\|\nabla\left(V-V_{n}\right)\right\|_{4 q /(4-q)} \rightarrow$ $0,\left\|D^{2}\left(V-V_{n}\right)\right\|_{q} \rightarrow 0$ and $\left\|\nabla\left(\Pi-\Pi_{n}\right)\right\|_{q} \rightarrow 0$. On the other hand, since inequality (6.9) was shown to be valid for $\phi \in C_{0}^{\infty}\left(\bar{\Omega}^{c}\right)^{3}$, we see that the sequence $\left(V_{n}\right)$ converges in $L^{p_{1}}\left(\bar{\Omega}^{c}\right)^{3},\left(\nabla V_{n}\right)$ in $L^{p_{2}}\left(\bar{\Omega}^{c}\right)^{9},\left(|\varrho|^{\kappa} V_{n}\right)$ in $L^{p_{3}}\left(\bar{\Omega}^{c}\right)^{3}$ if $\varrho \neq 0,\left(D^{2} V_{n} \mid \Omega_{R}\right)$ in $L^{q}\left(\Omega_{R}\right)^{27}$ and $\left(\nabla \Pi_{n}\right)$ in $L^{q}\left(\bar{\Omega}^{c}\right)^{3}$. Since $L^{p}$-convergence implies pointwise convergence a. e. of a subsequence, we may now conclude that the limit functions of the preceding sequences are $V, \nabla V,|\varrho|^{\kappa} V, D^{2} V \mid \Omega_{R}$ and $\nabla \Pi$, respectively. Thus inequality (6.9) remains valid when $\phi$ is replaced by $G$. This proves the theorem.

Theorem 6.2 Let $p \in(4,6), \lambda \in K_{\tau}$ and $G \in C_{0}^{\infty}\left(\bar{\Omega}^{c}\right)^{3}$. Then, for some $c_{0} \in \mathbb{R}$, we have

$$
\|\mathfrak{V}(\lambda, G)\|_{p}+\|\nabla \mathfrak{V}(\lambda, G)\|_{2}+\left\|\Pi(\lambda, G)+c_{0}\right\|_{2} \leq \mathfrak{C}(p)\left\|G \mid \bar{\Omega}^{c}\right\|_{-1,2} .
$$

Proof: We again proceed by contradiction. Suppose there is no constant $C_{0}>0$ such that

$$
\|\mathfrak{V}(\varrho, \phi)\|_{p}+\|\nabla \mathfrak{V}(\varrho, \phi)\|_{2}+\|\Pi(\varrho, \phi)+c(\varrho, \phi)\|_{2} \leq C_{0}\left\|\phi \mid \bar{\Omega}^{c}\right\|_{-1,2}
$$

for $\varrho \in K_{\tau}, \phi \in C_{0}^{\infty}\left(\bar{\Omega}^{c}\right)^{3}$, where $c(\varrho, \phi) \in \mathbb{C}$ is chosen in such a way that $\Pi(\varrho, \phi)+c(\varrho, \phi) \in$ $L^{r}\left(\bar{\Omega}^{c}\right)$ for any $r \in(3 / 2, \infty)$; see Corollary 6.3. Then, for any $n \in \mathbb{N}$, there exists $\varrho_{n} \in K_{\tau}$ and $\phi_{n} \in C_{0}^{\infty}\left(\bar{\Omega}^{c}\right)^{3}$ such that $\gamma_{n} \geq n\left\|\phi_{n} \mid \bar{\Omega}^{c}\right\|_{-1,2}$, where $\gamma_{n}:=\left\|\mathfrak{V}\left(\varrho_{n}, \phi_{n}\right)\right\|_{p}+$ $\left\|\nabla \mathfrak{V}\left(\varrho_{n}, \phi_{n}\right)\right\|_{2}+\left\|\Pi\left(\varrho_{n}, \phi_{n}\right)+c\left(\varrho_{n}, \phi_{n}\right)\right\|_{2}$. Note that $\gamma_{n}<\infty$ for $n \in \mathbb{N}$ by the choice of $c\left(\varrho_{n}, \phi_{n}\right)$ and by Corollary 6.1 and 6.2. We define

$$
v_{n}:=\gamma_{n}^{-1} \mathfrak{V}\left(\varrho_{n}, \phi_{n}\right), \sigma_{n}:=\gamma_{n}^{-1}\left(\Pi\left(\varrho_{n}, \phi_{n}\right)+c\left(\varrho_{n}, \phi_{n}\right)\right), g_{n}:=\gamma_{n}^{-1} \phi_{n} \quad \text { for } n \in \mathbb{N} .
$$

Then, as in the proof of Theorem 6.1, the pair $\left(v_{n}, \varrho_{n}\right)$ satisfies $(6.10)(n \in \mathbb{N})$. The regularity properties listed in Corollary 6.1 in the case $\varrho_{n} \neq 0$ and in Corollary 6.2 if $\varrho_{n}=0$ are valid for $v_{n}, \sigma_{n}$ in the place of $V$ and $\Pi$, respectively. Moreover Corollary 6.3 yields $\sigma_{n} \in L^{r}\left(\bar{\Omega}^{c}\right)^{3}$ and $v_{n, j}, \sigma_{n} \in C^{\infty}\left(\bar{\Omega}^{c}\right)$ for $r \in(3 / 2, \infty), n \in \mathbb{N}, 1 \leq j \leq 3$. The inequality $\gamma_{n} \geq n\left\|\phi_{n} \mid \bar{\Omega}^{c}\right\|_{-1,2}$ implies that

$$
\left\|v_{n}\right\|_{p}+\left\|\nabla v_{n}\right\|_{2}+\left\|\sigma_{n}\right\|_{2}=1 \geq n\left\|g_{n} \mid \bar{\Omega}^{c}\right\|_{-1,2} \quad \text { for } n \in \mathbb{N} .
$$

As a first consequence of (6.14), we note that $\left\|g_{n} \mid \bar{\Omega}^{c}\right\|_{-1,2} \rightarrow 0$. Fix some function $\zeta \in C_{0}^{\infty}\left(B_{S+1}\right)$ with $\zeta \mid B_{S}=1$. Put $B:=B_{S+1} \backslash \overline{B_{S}}$. Since $v_{n} \mid \partial \Omega=0$ according to (6.10), and because $p>2, v_{n} \in L^{p}\left(\bar{\Omega}^{c}\right)^{3}$ and $\nabla v_{n} \in L^{2}\left(\bar{\Omega}^{c}\right)^{9}$, it follows that $\zeta v_{n} \mid \Omega_{S+1} \in$ $W_{0}^{1,2}\left(\Omega_{S+1}\right)^{3}$ and $\left\|\zeta v_{n} \mid \Omega_{S+1}\right\|_{1,2} \leq \mathfrak{C}(p)\left(\left\|v_{n}\right\|_{p}+\left\|\nabla v_{n}\right\|_{2}\right)$, and similarly $\left\|v_{n} \mid B\right\|_{1,2} \leq$ $\mathfrak{C}(p)\left(\left\|v_{n}\right\|_{p}+\left\|\nabla v_{n}\right\|_{2}\right)$, for $n \in \mathbb{N}$. These observations, (6.14) and Theorem 2.3 allow us to choose a subsequence of $\left(\left(v_{n}, \sigma_{n}, \varrho_{n}\right)\right)$, also denoted by $\left(\left(v_{n}, \sigma_{n}, \varrho_{n}\right)\right)$, with the following properties: $v_{n} \rightarrow V$ in $L^{p}\left(\bar{\Omega}^{c}\right)^{3}$ for some $V \in L^{p}\left(\bar{\Omega}^{c}\right)^{3}, \partial_{l} v_{n} \rightarrow V^{(l)}$ in $L^{2}\left(\bar{\Omega}^{c}\right)^{3}$ for some $V^{(l)} \in L^{2}\left(\bar{\Omega}^{c}\right)^{3}(1 \leq l \leq 3), \zeta v_{n} \mid \Omega_{S+1} \rightarrow Z$ in $W_{0}^{1,2}\left(\Omega_{S+1}\right)^{3}$ for some $Z \in W_{0}^{1,2}\left(\Omega_{S+1}\right)^{3}$, $\left\|v_{n} \mid B-Y\right\|_{2} \rightarrow 0$ for some $Y \in L^{2}(B)^{3}, \sigma_{n} \rightarrow \Pi$ in $L^{2}\left(\bar{\Omega}^{c}\right)^{3}$ for some $\Pi \in L^{2}\left(\bar{\Omega}^{c}\right)^{3}$, $\left\|\sigma_{n} \mid B-\Gamma\right\|_{-1,2} \rightarrow 0$ for some $\Gamma \in L^{2}(B)$, and $\varrho_{n} \rightarrow \varrho$ in $\mathbb{C}$ for some $\varrho \in K_{\tau}$. 
By the reasoning indicated in the proof of Theorem 6.1, we may conclude that $V \in$ $W_{\text {loc }}^{1,1}\left(\bar{\Omega}^{c}\right)^{3}, \partial_{l} V=V^{(l)}$ for $1 \leq l \leq 3, \zeta V\left|\Omega_{S+1}=Z, V\right| B=Y, \Pi \mid B=\Gamma$. Therefore $\nabla V \in L^{2}\left(\bar{\Omega}^{c}\right)^{9},\left\|v_{n}-V \mid B\right\|_{2} \rightarrow 0$ and $\left\|\sigma_{n}-\Pi \mid B\right\|_{-1,2} \rightarrow 0$. Since $\zeta V \mid \Omega_{S+1}=Z \in$ $W_{0}^{1,2}\left(\Omega_{S+1}\right)^{3}, \zeta \mid B_{S}=1$ and $\bar{\Omega} \subset B_{S}$, we get $V \mid \partial \Omega=0$. Moreover, recalling that $\partial_{l} V=$ $V^{(l)}$ for $1 \leq l \leq 3$ and $\left\|g_{n} \mid \bar{\Omega}^{c}\right\|_{-1,2} \rightarrow 0$, we may deduce from (6.10) that $\operatorname{div} V=0$ and that (3.1) as well as (3.2) hold with $A=\bar{\Omega}^{c}, G=0$ and $\lambda=\varrho$. Since $V \in L^{p}\left(\bar{\Omega}^{c}\right)^{3}$ and $\nabla V \in L^{2}\left(\bar{\Omega}^{c}\right)^{9}$, we may at this point apply Theorem 5.2 to obtain $V=0$. Now (3.2) yields $\int_{\bar{\Omega}^{c}} \Pi \operatorname{div} \psi=0$ for $\psi \in C_{0}^{\infty}\left(\bar{\Omega}^{c}\right)^{3}$, implying that $\Pi \in W_{l o c}^{1,1}\left(\bar{\Omega}^{c}\right)^{3}$ with $\nabla \Pi=0$. As a consequence $\Pi=c$ a. e. for some $c \in \mathbb{C}$ (Corollary 2.2). But $\Pi \in L^{2}\left(\bar{\Omega}^{c}\right)$, so $\Pi=0$. In this way we arrive at the relations $\left\|v_{n} \mid B\right\|_{2} \rightarrow 0$ and $\left\|\sigma_{n} \mid B\right\|_{-1,2} \rightarrow 0$.

On the other hand we may apply (6.1) with $V$, $\Pi$ replaced by $v_{n}=\mathfrak{V}\left(\varrho_{n}, \gamma_{n}^{-1} \phi_{n}\right), \sigma_{n}=$ $\Pi\left(\varrho_{n}, \gamma_{n}^{-1} \phi_{n}\right)+\gamma_{n}^{-1} c\left(\varrho_{n}, \phi_{n}\right)$, to obtain

$$
\left\|v_{n}\right\|_{p}+\left\|\nabla v_{n}\right\|_{2}+\left\|\sigma_{n}\right\|_{2} \leq \mathfrak{C}(p)\left(\left\|g_{n}\left|\bar{\Omega}^{c}\left\|_{-1,2}+\right\| v_{n}\right| B\right\|_{2}+\left\|\varrho_{n} \mid B\right\|_{-1,2}\right) \quad \text { for } n \in \mathbb{N} .
$$

It follows that the left-hand side of this estimate tends to zero for $n \rightarrow \infty$, which is a contradiction to (6.14). As a consequence, there must be a constant $C_{0}>0$ such that (6.13) holds. This proves Theorem 6.2.

$\left\langle\right.$ theoremT7.40〉 Theorem 6.3 Let $\lambda \in K_{\tau}$ and $G \in \mathcal{D}_{0}^{-1,2}\left(\bar{\Omega}^{c}\right)^{3}$. Then there is a unique function $V:=$ $\widetilde{\mathfrak{V}}(\lambda, G) \in \mathcal{D}_{0}^{1,2}\left(\bar{\Omega}^{c}\right)^{3}$ such that $\operatorname{div} V=0$,

$$
\int_{\bar{\Omega}^{c}}\left(\nabla V \cdot \nabla \psi+\tau \partial_{1} V \psi+\lambda V \cdot \psi\right) d x=G(\psi) \quad \text { for } \psi \in C_{0}^{\infty}\left(\bar{\Omega}^{c}\right)^{3} \text { with div } \psi=0 .(6.15) \mathrm{T7.40.10}
$$

This function $V$ satisfies the inequality $\|V\|_{p}+\|\nabla V\|_{2} \leq \mathfrak{C}(p)\|G\|_{-1,2}$ for $p \in(4,6]$.

Proof: Since $\mathcal{D}_{0}^{1,2}\left(\bar{\Omega}^{c}\right)^{3} \subset L^{6}\left(\bar{\Omega}^{c}\right)^{3}, \nabla W \in L^{2}\left(\bar{\Omega}^{c}\right)^{9}$ and $W \mid \partial \Omega=0$ for $W \in \mathcal{D}_{0}^{1,2}\left(\bar{\Omega}^{c}\right)^{3}$, the uniqueness statement in Theorem 6.3 follows from Theorem 5.2. Concerning existence, Theorem 2.6 yields a sequence $\left(\phi_{n}\right)$ in $C_{0}^{\infty}\left(\bar{\Omega}^{c}\right)^{3}$ with $\left\|G-\phi_{n}\right\|_{-1,2} \rightarrow 0$. Therefore, by the estimate in Theorem 2.5 and (6.12), the sequence $\left(\mathfrak{V}\left(\lambda, \phi_{n}\right)\right)$ converges in $L^{6}\left(\bar{\Omega}^{c}\right)^{3}$, and the sequence $\left(\nabla \mathfrak{V}\left(\lambda, \phi_{n}\right)\right)$ in $L^{2}\left(\bar{\Omega}^{c}\right)^{9}$. Thus there is a function $V:=\widetilde{\mathfrak{V}}(\lambda, G) \in$ $L^{6}\left(\bar{\Omega}^{c}\right)^{3} \cap W_{\text {loc }}^{1,1}\left(\bar{\Omega}^{c}\right)^{3}$ with $\nabla V \in L^{2}\left(\bar{\Omega}^{c}\right)^{9}$ such that $\left\|V-\mathfrak{V}\left(\lambda, \phi_{n}\right)\right\|_{6} \rightarrow 0$ and $\| \nabla V-$ $\nabla \mathfrak{V}\left(\lambda, \phi_{n}\right) \|_{2} \rightarrow 0$. Then $\left\|V-\mathfrak{V}\left(\lambda, \phi_{n}\right) \mid \Omega_{S}\right\|_{1,2} \rightarrow 0$, so $V \mid \partial \Omega=0$. We thus have found that $V \in \mathcal{D}_{0}^{1,2}\left(\bar{\Omega}^{c}\right)^{3}$. The relations $\left\|V-\mathfrak{V}\left(\lambda, \phi_{n}\right)\right\|_{6} \rightarrow 0$ and $\left\|\nabla V-\nabla \mathfrak{V}\left(\lambda, \phi_{n}\right)\right\|_{2} \rightarrow 0$ and the fact that $\mathfrak{V}\left(\lambda, \phi_{n}\right)$ satisfies (1.8) with $\phi_{n}$ in the place of $G$ imply (6.15). Again referring to (6.12), we get $\left\|\mathfrak{V}\left(\lambda, \phi_{n}\right)\right\|_{p}+\left\|\nabla \mathfrak{V}\left(\lambda, \phi_{n}\right)\right\|_{2} \leq \mathfrak{C}\left\|\phi_{n} \mid \bar{\Omega}^{c}\right\|_{-1,2}$ for $n \in \mathbb{N}$ and $p \in(4,6)$. In view of the estimate in Theorem 2.5 , the preceding inequality also holds for $p=6$. These observations imply the estimate stated in Theorem 6.3.

As an immediate consequence of Theorem 2.6, 5.2 and the regularity properties of solutions to (1.8) mentioned in Corollary 6.1, 6.2 and in Theorem 6.3, we obtain that the functions $\mathfrak{V}(\lambda, G)$ and $\widetilde{\mathfrak{V}}(\lambda, G)$ coincide if $G$ has suitable regularity:

Corollary 6.4 Let $\lambda \in K_{\tau}$ and $p \in(1, \infty)$, with either $\lambda \neq 0$ or $p<2$. Let $G \in$ $L^{p}\left(\bar{\Omega}^{c}\right)^{3} \cap \mathcal{D}_{0}^{-1,2}\left(\bar{\Omega}^{c}\right)^{3}$. Then the function $\mathfrak{V}(\lambda, G)$, as defined in Corollary $6.1(\lambda \neq 0)$ or 6.2 $(\lambda=0)$, and the function $\widetilde{\mathfrak{V}}(\lambda, G)$ introduced in Theorem 6.3 coincide. 
We add a technical remark which is relevant for the proof of the ensuing theorem, which is another one of our main results.

$\langle$ lemmaL7.10〉

Lemma 6.1 Let $\lambda \in K_{\tau}, p \in(1,2)$ in the case $\lambda=0, p \in(1, \infty)$ if $\lambda \neq 0, G \in L^{p}\left(\bar{\Omega}^{c}\right)^{3}$. Then $\mathfrak{V}(\lambda, G)=\mathfrak{V}\left(\lambda, \mathcal{P}_{p}(G)\right)$.

Proof: For $\psi \in C_{0}^{\infty}\left(\bar{\Omega}^{c}\right)^{3}$ with $\operatorname{div} \psi=0$, we have $\int_{\bar{\Omega}^{c}} G \cdot \psi d x=\int_{\bar{\Omega}^{c}} \mathcal{P}_{p}(G) \cdot \psi d x$ (second and forth equation in Corollary 2.5). Therefore (3.1) is satisfied with $V=$ $\mathfrak{V}(\lambda, G)$ and $V=\mathfrak{V}\left(\lambda, \mathcal{P}_{p}(G)\right)$. Thus, due to the integrability properties of $\mathfrak{V}(\lambda, G)$ and $\mathfrak{V}\left(\lambda, \mathcal{P}_{p}(G)\right)$ mentioned in Corollary $6.1(\lambda \neq 0)$ and $6.2(\lambda=0)$, and because $\mathfrak{V}(\lambda, G)\left|\partial \Omega=\mathfrak{V}\left(\lambda, \mathcal{P}_{p}(G)\right)\right| \partial \Omega=0$. we may apply Theorem 5.2 , which yields $\mathfrak{V}(\lambda, G)=$ $\mathfrak{V}\left(\lambda, \mathcal{P}_{p}(G)\right)$.

$\left\langle\right.$ theoremT7.30〉 Theorem 6.4 For $p \in(1, \infty)$, the estimate $\|\mathfrak{V}(\lambda, G)\|_{p} \leq \mathfrak{C}(p)|\lambda|^{-2}\|G\|_{p}$ holds for $G \in$ $L^{p}\left(\bar{\Omega}^{c}\right)^{3}, \lambda \in K_{\tau} \backslash\{0\}$.

Proof: Let $\lambda \in K_{\tau} \backslash\{0\}$. For $p \in(1, \infty)$, put $D_{p}:=H_{p}\left(\bar{\Omega}^{c}\right) \cap W^{2, p}\left(\bar{\Omega}^{c}\right)^{3} \cap W_{0}^{1, p}\left(\bar{\Omega}^{c}\right)^{3}$. We will apply some of our preceding results both for $\mu=1$ and $\mu=-1$, with $\mu$ being the parameter fixing the sign of the Oseen term in (1.8), (3.1), (3.2) and (3.5). Therefore we have this parameter appear explicitly in our notation, denoting by $\left(\mathfrak{V}^{(\mu)}(\lambda, G), \Pi^{(\mu)}(\lambda, G)\right)$ the solution of (1.8) introduced in Corollary 6.1, for $p \in(1, \infty), G \in L^{p}\left(\bar{\Omega}^{c}\right)^{3}, \mu \in\{-1,1\}$. In addition we set $\mathfrak{A}_{p}^{(\mu)}(V):=\mathcal{P}_{p}\left(-\Delta V+\mu \tau \partial_{1} V+\lambda V\right)$ for $V \in D_{p}$.

Let $\mu \in\{-1,1\}, p \in(1, \infty), G \in H_{p}\left(\bar{\Omega}^{c}\right)$, and abbreviate $V:=\mathfrak{V}^{(\mu)}(\lambda, G), \Pi:=$ $\Pi^{(\mu)}(\lambda, G)$. Then $V \in W^{2, p}\left(\bar{\Omega}^{c}\right)^{3}$, and the pair $(V, \Pi)$ is a solution of (1.8); see Corollary 6.1. In particular we have $V \mid \partial \Omega=0$ (hence $\left.V \in W_{0}^{1, p}\left(\bar{\Omega}^{c}\right)^{3}\right)$ and $\operatorname{div} V=0$. Thus Theorem 2.2 implies in particular that $V \in H_{p}\left(\bar{\Omega}^{c}\right)$. These observations mean that $V \in D_{p}$. Since $\nabla \Pi \in L^{p}\left(\bar{\Omega}^{c}\right)^{3}$ and the pair $(V, \Pi)$ solves (1.8), it follows with the third equation in Corollary 2.5 that $\mathfrak{A}_{p}^{(\mu)}(V)=\mathcal{P}_{p}(G)$. But $G \in H_{p}\left(\bar{\Omega}^{c}\right)$, so $\mathfrak{A}_{p}^{(\mu)}(V)=G$ again by Corollary 2.5. We have thus shown that $\mathfrak{A}_{p}^{(\mu)}: D_{p} \mapsto H_{p}\left(\bar{\Omega}^{c}\right)$ is onto, with

$$
\mathfrak{A}_{p}^{(\mu)}\left(\mathfrak{V}^{(\mu)}(\lambda, G)\right)=G \text { for } G \in H_{p}\left(\bar{\Omega}^{c}\right) .
$$

Let $V \in D_{p}$ with $\mathfrak{A}_{p}^{(\mu)}(V)=0$. Obviously $-\Delta V+\mu \tau \partial_{1} V+\lambda V \in L^{p}\left(\bar{\Omega}^{c}\right)^{3}$, so we may put $\Pi:=-\mathcal{G}_{p}\left(-\Delta V+\mu \tau \partial_{1} V+\lambda V\right)$. Note that $\Pi \in W_{l o c}^{1, p}\left(\bar{\Omega}^{c}\right)$ with $\nabla \Pi \in L^{p}\left(\bar{\Omega}^{c}\right)^{3}$ by Theorem 2.11. This latter reference and the assumption $\mathfrak{A}_{p}^{(\mu)}(V)=0$ imply the pair $(V, \Pi)$ is a solution of (3.5) with $G=0$. Since $V \in D_{p} \subset W_{0}^{1, p}\left(\bar{\Omega}^{c}\right)^{3}$, we additionally have $V \mid \partial \Omega=0$, so the pair is a solution of (1.8) with $G=0$. Theorem 5.2 now yields $V=0$. Thus the operator $\mathfrak{A}_{p}^{(\mu)}$ is one-to-one. At this point we have found that $\mathfrak{A}_{p}^{(\mu)}: D_{p} \mapsto H_{p}\left(\bar{\Omega}^{c}\right)$ is bijective, with (6.16) implying

$$
\left(\mathfrak{A}_{p}^{(\mu)}\right)^{-1}(G)=\mathfrak{V}^{(\mu)}(\lambda, G) \text { for } G \in H_{p}\left(\bar{\Omega}^{c}\right) .
$$

This latter equation, the fact that $\mathfrak{V}^{(\mu)}(\lambda, G) \in D_{p}$, and Theorem 3.7 yield that $\left(\mathfrak{A}_{p}^{(\mu)}\right)^{-1}$ : $H_{p}\left(\bar{\Omega}^{c}\right) \mapsto H_{p}\left(\bar{\Omega}^{c}\right)$ is bounded.

Let us write $\mathfrak{A}^{+}, \mathfrak{A}^{-}$instead of $\mathfrak{A}^{(1)}$ and $\mathfrak{A}^{(-1)}$, respectively. The notation $\mathfrak{V}^{+}(\lambda, G)$ and $\mathfrak{V}^{-}(\lambda, G)$ are to be understood in an analogous way, for $G \in L^{p}\left(\bar{\Omega}^{c}\right)^{3}, p \in(1, \infty)$. 
Integrations by parts and the second and forth equation in Corollary 2.5 yield $\int \bar{\Omega}^{c} \mathfrak{A}_{p}^{+}(V)$. $W d x=\int_{\bar{\Omega}} V \cdot \mathfrak{A}_{p}^{-}(W) d x$ for $p \in(1, \infty), V \in D_{p}, W \in D_{p^{\prime}}$, and for $p \in(1, \infty)$. It follows again with the second and forth equation in Corollary 2.5 that the operator $\left(\mathfrak{A}_{p^{\prime}}^{-}\right)^{-1} \circ \mathcal{P}_{p^{\prime}}$ is dual in $L^{p}\left(\bar{\Omega}^{c}\right)^{3}$ to $\left(\mathfrak{A}_{p}^{+}\right)^{-1} \circ \mathcal{P}_{p}(p \in(1, \infty))$.

Let the operator norm of linear bounded operators $\mathfrak{F}: L^{p}\left(\bar{\Omega}^{c}\right)^{3} \mapsto L^{p}\left(\bar{\Omega}^{c}\right)^{3}$ be denoted by $\left\||\||_{p}\right.$, that is, $\| \mid \mathfrak{F} \|_{p}:=\sup \left\{\|\mathfrak{F}(V)\|_{p} /\|V\|_{p}: V \in L^{p}\left(\bar{\Omega}^{c}\right)^{3}, V \neq 0\right\}$, for $p \in(1, \infty)$. Then a functional analytical principle allows us to conclude that

$$
\left.\left\|\left|\left(\mathfrak{A}_{p}^{+}\right)^{-1} \circ \mathcal{P}_{p}\left\|\left.\right|_{p}=\right\|\right|\left(\mathfrak{A}_{p^{\prime}}^{-}\right)^{-1} \circ \mathcal{P}_{p^{\prime}}\right\|\right|_{p^{\prime}} \quad(p \in(1, \infty)) .
$$

Now let $p \in(2, \infty)$. Then $p^{\prime} \in(1,2)$, so by Theorem 6.1 with $q=p_{3}=p^{\prime}$ we know that $\left\|\mathfrak{V}^{-}(\lambda, G)\right\|_{p^{\prime}} \leq \mathfrak{C}\left(p^{\prime}\right)|\lambda|^{-2}\|G\|_{p^{\prime}}$ for $G \in L^{p^{\prime}}\left(\bar{\Omega}^{c}\right)^{3}$. Using (2.8) and (6.17), we may conclude that $\left\|\left.\left|\left(\mathfrak{A}_{p^{\prime}}^{-}\right)^{-1} \circ \mathcal{P}_{p^{\prime}}\|\|_{p^{\prime}} \leq \mathfrak{C}\left(p^{\prime}\right)\right| \lambda\right|^{-2}\right.$. In view of (6.18), we thus obtain $\left\|\left|\left(\mathfrak{A}_{p}^{+}\right)^{-1} \circ \mathcal{P}_{p} \|\right|_{p} \leq \mathfrak{C}(p)|\lambda|^{-2}\right.$. Here $p$ was arbitrarily taken from $(2, \infty)$. If $p \in(1,2)$, the preceding inequality follows directly from Theorem $6.1,(6.17)$ and $(2.8)$. In order to handle the case $p=2$, we interpolate between $L^{3}\left(\bar{\Omega}^{c}\right)^{3}$ and $L^{3 / 2}\left(\bar{\Omega}^{c}\right)^{3}$. To this end, we note that by (6.17), Lemma 6.1 and the first equation in Corollary 2.5, we get $\left(\mathfrak{A}_{3}^{+}\right)^{-1}\left(\mathcal{P}_{3}(G)\right)=$ $\mathfrak{V}^{+}\left(\lambda, \mathcal{P}_{3}(G)\right)=\mathfrak{V}^{+}(\lambda, G)=\mathfrak{V}^{+}\left(\lambda, \mathcal{P}_{3 / 2}(G)\right)=\left(\mathfrak{A}_{3 / 2}^{+}\right)^{-1}\left(\mathcal{P}_{3 / 2}(G)\right)$ for $G \in L^{3}\left(\bar{\Omega}^{c}\right)^{3} \cap$ $L^{3 / 2}\left(\bar{\Omega}^{c}\right)^{3}$. Since we showed that the estimate $\left\|\left|\left(\mathfrak{A}_{p}^{+}\right)^{-1} \circ \mathcal{P}_{p} \|\right|_{p} \leq \mathfrak{C}(p)|\lambda|^{-2}\right.$ is valid for $p \in(1, \infty) \backslash\{2\}$, we may now conclude by the Riesz-Thorin interpolation theorem that it holds for $p=2$ as well. Thus we have proved this estimate for any $p \in(1, \infty)$. Combining it with Lemma 6.1 and (6.17), we finally get $\left\|\mathfrak{V}^{+}(\lambda, G)\right\|_{p}=\left\|\mathfrak{V}^{+}\left(\lambda, \mathcal{P}_{p}(G)\right)\right\|_{p}=$ $\left\|\left(\mathfrak{A}_{p}^{+}\right)^{-1}\left(\mathcal{P}_{p}(G)\right)\right\|_{p} \leq \mathfrak{C}(p)|\lambda|^{-2}\|G\|_{p}$ for $G \in L^{p}\left(\bar{\Omega}^{c}\right)^{3}, p \in(1, \infty)$.

\section{References}

AR [1] C. Amrouche and U. Razafison, On the Oseen problem in three-dimensional exterior domains, Anal. Appl. 4 (2006), 133-162.

$\mathrm{Bab}[2]$ K. I. BABEnko, Spectrum of the linearized problem of flow of a viscous incompressible liquid round a body, Sov. Phys. Dokl. 27 (1982), 25-27.

BoSo2 [3] W. Borchers AND H. Sohr, On the semigroup of the Stokes operator for exterior domains in $L^{q}$-spaces, Math. Z. 196 (1987), 415-425.

BoSo [4] W. Borchers AND H. Sohr, On the equations $\operatorname{rot} v=g$ and $\operatorname{div} v=f$ with zero boundary conditions, Hokkaido Math. J. 19 (1990), 67-87.

De1R [5] P. DeuRING, An integral operator related to the Stokes system in exterior domains, Math. Methods Appl. Sci. 13 (1990), 323-333. Addendum, Math. Methods Appl. Sci. 14 (1991), 445.

De2R [6] P. Deuring, The resolvent problem for the Stokes system in exterior domains: an elementary approach, Math. Meth. in the Appl. Sci. 13 (1990), 335-349.

De3R [7] P. Deuring, The Stokes system in exterior domains: L $^{p}$-estimates for small values of a resolvent parameter, J. Appl. Math. Phys. (ZAMP) 41 (1990), 829-842. 
D-DCDSA [8] P. Deuring, Pointwise spatial decay of time-dependent Oseen flows: the case of data with noncompact support. Discr. Cont. Dyn. Syst. Ser. A 33 (2013), 2757-2776.

De-stab [9] P. Deuring, Stability of stationary viscous incompressible flow around a rigid body performing a translation, submitted.

D-JMFM [10] P. Deuring, Pointwise spatial decay of weak solutions to the Navier-Stokes system in 3D exterior domains. J. Math. Fluid Mech. 17 (2015), 199-232.

DN [11] P. Deuring And J. Neustupa, An eigenvalue criterion for stability of a steady Navier-Stokes flow in $\mathbb{R}^{3}$, J. Math. Fluid Mech. 12 (2010), 202-240.

DV [12] P. Deuring and W. Varnhorn, On Oseen resolvent estimates, Diff. Int. Equat. 23 (2010), 1139-1149.

D-ZAmp [13] P. Deuring, S. KraČmar and Š. NeČasová, Asymptotic structure of viscous incompressible flow around a rotating body, with nonvanishing flow field at infinity. To appear in Z. Angew. Math. Phys. 68 (2017).

Faneu2008 [14] R. FARWig AND J. Neustupa, On the spectrum of an Oseen-type operator arising from flow around a rotating body, Int. Equ. Oper. Theory 62 (2008), 169-189.

FM [15] D. Fujiwara and H. Morimoto, An $L_{r}$-theorem of the Helmholtz decomposition of vector fields, J. Fac. Sci. Univ. Tokyo (1A) 24 (1977), 685-700.

FJK [16] S. Fučík, O. John And A. Kufner, Function Spaces, Noordhoff, Leyden, 1977.

Galdineu [17] G. P. GALDI, An Introduction to the Mathematical Theory of the Navier-Stokes Equations. Steady-State Problems, 2nd edition, Springer, New York e.a., 2011.

Gi [18] Y. GigA, Analyticity of the semigroup generated by the Stokes operator in $L^{r}$ spaces, Math. Z. 178 (1981), 297-329.

KS [19] T. Kobayashi and Y. Shibata, On the Oseen equation in the three dimensional exterior domains, Math. Ann. 310 (1998), 1-45.

KSo [20] H. Kozono And H. Sohr, New a priori estimates for the Stokes equations in exterior domains, Indiana Univ. Math. J. 40 (1991), 1-27.

Miya [21] T. MryakaWA, On nonstationary solutions of the Navier-Stokes equations in an exterior domain, Hiroshima Math. J. 12 (1982), 115-140.

Neri [22] U. NerI, Singular integrals, Lecture Notes in Mathematics 200, Springer, Berlin e.a., 1971.

SiSo2 [23] C. G. Simader And H. Sohr, A new approach to the Helmholtz decomposition and the Neumann problem in $L^{q}$-spaces for bounded and exterior domains, Mathematical problems relating to the Navier-Stokes equation, 1-35, Ser. Adv. Math. Appl. Sci. 11, World Sci. Publ., River Edge, NJ, 1992. 
SiSo [24] C. G. Simader And H. Sohr, The Dirichlet problem for the Laplacian in bounded and unbounded domains, Pitman Resarch Notes in Mathematics 360, Addison Wesley Longman, Harlow, 1996.

Sol [25] V. A. Solonnikov, On estimates of solutions of the non-stationary Stokes problem in anisotropic Sobolev spaces and on estimates for the resolvent of the Stokes operator. Uspekhi Mat. Nauk 58 (2003), 123-156 (Russian); English translation, Russian Math. Surveys 58 (2003), 331-365. 